Article

\title{
Food Sources of Energy and Nutrients of Public Health Concern and Nutrients to Limit with a Focus on Milk and other Dairy Foods in Children 2 to 18 Years of Age: National Health and Nutrition Examination Survey, 2011-2014
}

\author{
Carol E. O'Neil ${ }^{1, *}$, Theresa A. Nicklas ${ }^{2}$ and Victor L. Fulgoni III ${ }^{1,3}$ (i) \\ 1 Louisiana State University Agricultural Center (Emeritus), School of Nutrition and Food Science, \\ 143 Kenilworth Parkway, Baton Rouge, LA 70808, USA; VIC3rd@aol.com \\ 2 USDA/ARS/CNRC, 1100 Bates St., Baylor College of Medicine, Houston, TX 77030, USA; tnicklas@bcm.edu \\ 3 Nutrition Impact, LLC, 9725 D Drive North, Battle Creek, MI 49014, USA \\ * Correspondence: coneil8@cox.net
}

Received: 3 July 2018; Accepted: 2 August 2018; Published: 9 August 2018

\begin{abstract}
Many children are not meeting current nutrient recommendations. The objective of this study was to determine the food sources of energy, nutrients of public health concern, and nutrients to limit with a focus on dairy foods. Twenty-four-hour dietary recall data from children $2-5(n=1511)$, 6-11 $(n=2193)$, and 12-18 years $(n=2172)$ participating in NHANES 2011-2014 were analyzed. Energy, fiber, calcium, potassium, vitamin D, added sugars, saturated fatty acids (SFA), and sodium intakes were sample-weighted and ranked on percentage contribution to the diet using specific food group intake and disaggregated data for dairy foods. For children 2-5, 6-11, and 12-18 years, milk, sweet bakery products, and sweetened beverages, respectively were the top food sources of energy, respectively. For calcium, potassium, and vitamin D, milk was the top ranked food source in all age groups. For children $2-5,6-11$, and $12-18$ years, milk, sweet bakery products, and pizza, respectively were the top three ranked food sources of SFA; and sugar sweetened beverages and sweet bakery products were to top two food group sources of added sugars. Cured meats/poultry, pizza, and pizza, respectively, were the top ranked food sources of sodium for the three age groups. Identification of food sources of these nutrients can help health professionals implement appropriate dietary recommendations and plan age-appropriate interventions.
\end{abstract}

Keywords: NHANES; energy intakes; nutrients; children; adolescents; dietary sources; dairy foods

\section{Introduction}

Dairy products are rich in three of the four nutrients of public health concern: calcium, vitamin D, and potassium [1]. The 2015-2020 Dietary Guidelines Advisory Committee (DGAC) determined that several nutrients: vitamins A, E, and C; folate; magnesium; and iron (in adolescent females) were under consumed relative to the Estimated Average Requirement (EAR) or Adequate Intake (AI) levels set by the Institute of Medicine and these were characterized as "shortfall nutrients". The DGAC confirmed that fiber, calcium, vitamin $\mathrm{D}$, and potassium remained nutrients of public health concern since underconsumption has been linked to adverse health outcomes [2]. In children 2-18 years of age (years), milk has previously been shown to be the primary source of calcium, vitamin $\mathrm{D}$, and potassium [3]. Dairy products, especially milk and yogurt, also provide protein, saturated fatty acids (SFA), riboflavin, vitamin $B_{12}$, and phosphorus. 
Modeling techniques with data from the National Health and Nutrition Examination Survey (NHANES) have shown that the prevalence of inadequate calcium and potassium intakes could be reduced if additional servings of dairy foods were consumed [4,5]. However, per captia consumption of fluid milk has declined sharply since 1975, when it was 247 pounds per person, to 154 pounds per person in 2016. The lack of nutrients from fluid milk has been partially offset by an increase in cheese and yogurt consumption over this time span [6].

Although dairy foods contribute to shortfall nutrients, there is concern that these foods may also contribute high levels of energy, added sugars, from flavored milk and sweet dairy drinks; SFA; and sodium-all of which may lead to chronic diseases in older adolescents and adults $[7,8]$. There has been some speculation that plant-based drinks may provide health benefits over dairy foods. However, modeling studies, using NHANES 2007-2010 data, have shown that when comparing the usual intake of macronutrients and shortfall nutrients of three dietary scenarios that increased intake of: (1) plant-based foods; (2) protein-rich plant foods; and (3) dairy foods, including milk, cheese, and yogurt. The dairy model reduced the percent of children not meeting the EAR for calcium, vitamins A and D, magnesium, and protein, while sodium and SFA intakes increased [9]. Thus, it is very important to understand more fully the food sources that provide these important sources of nutrients in dairy; this can be done in part by disaggregating the data.

To help assuage nutrient shortfalls, the recommendation for daily dairy intake for children is age dependent: 2 cup equivalents (CE) for children 2-3 years of age (years), 2.5 CE for children 4-8 years, and $3 \mathrm{CE}$ for children 9-18 years [10]. In general, young children meet the recommendations for dairy; on average, males and females $2-5$ years consume 2.04 and $2.03 \mathrm{CE}$ of total dairy, respectively [11]. However, as children get older, consumption goes down, especially in females. Males and females 6-11 years consume 2.53 and $1.90 \mathrm{CE}$, respectively; and males and females 12-19 years consume 2.40 and 1.61 CE, respectively [11]. These data are concerning, especially for females, since although the recommendation for dairy intake does not change with gender, in general females need [12] and consume [13] less energy than males. In addition, it is concerning that consumption declines with age.

Understanding food sources of energy, shortfall nutrients, and nutrients to limit is important at any age, including children. Dietary influences and eating behaviors established in childhood play an important role in growth and development in children [14,15]. They also provide a reasonable basis for adult dietary preferences [16]. Although the majority of information linking diet to chronic disease is available for adults, there is some evidence suggesting that encouraging consumption of foods that provide shortfall nutrients while reducing nutrients to limit may reduce risk factors for chronic diseases, including cardiovascular disease (CVD) [17], hypertension [18], insulin sensitivity [19], obesity [20], and abdominal adiposity [21].

Identifying food sources, including mixed-dish foods—such as pizza or Mexican dishes-that provide energy, shortfall nutrients, and nutrients to limit can help nutrition educators design age-specific programs to help them modify food and nutrient intake [22,23]. Targeted nutrition education may have an indirect positive effect of increasing children's intakes of food groups that provide shortfall nutrients, while limiting foods that provide nutrients to limit, thus moving children closer to meeting dietary recommendations $[10,24]$. The purpose of this study was to examine food sources providing energy, shortfall nutrients, and nutrients to limit in three age groups of children using data from the NHANES 2011-2014. This is the first detailed list of food sources in children (2-18 years) since the NHANES 2003-2006 studies [3] and is the first to examine food sources in three age groups of children. Additionally, given milk, cheese, and yogurt are used as ingredients in many mixed dishes, which are not captured in a simple analyses of food sources of nutrients, this study also determined the nutrients from milk, cheese, and yogurt in mixed dishes thereby obtaining a more thorough contribution of dairy products to the diet of children. 


\section{Materials and Methods}

\subsection{Study Overview, Study Population, and Analytic Sample}

The NHANES is a program of studies designed to assess the health and nutritional status of free-living individuals in the US. Online information about the NHANES, including the purpose [25], plan and operations, sampling and weighting procedures, analytic guidelines [26], response rates, and population totals [27], is available. Data from children 2-18 years of age (years) participating in the NHANES from 2011 to 2014 were used for these analyses. The final analytic sample had 5876 participants; children were separated into three age groups: $2-5$ years $(n=1511), 6-11$ years $(n=2193)$, and 12-18 years $(n=2172)$. The National Center for Health Statistics (NCHS) Research Ethics Review Board has approved the use of human subjects for NHANES studies [28]; and further institutional review was not required.

\subsection{Dietary Intake}

Dietary intake data for the NHANES used in this study were obtained from the in-person 24-h dietary recall interview [29] using an Automated Multiple-Pass Method [30]. Although a second, telephone interview, was also taken 3 to 10 days after the in-person interview, only the in-person interview was used because of the difference in the methodology for collecting the two recalls. A single 24-h dietary recall administered in a large population can provide data to adequately estimate population mean intakes [31]. Survey participants 12 years and older completed their own dietary interview; children 6 to 11 years were assisted by an adult, usually a parent; and parents /guardians reported for children younger than 6 years [29].

\subsection{Food Groupings and Composition}

The relevant What We Eat in America (WWEIA), the dietary component of NHANES, food category classification systems [32] were used to classify all foods. The WWEIA food categories contain 15 main groups: milk and dairy; protein foods; mixed dishes; grains; snacks and sweets; fruit; vegetables; beverages, nonalcoholic; alcoholic beverages; water; fats and oils; condiments and sauces; sugars; infant formula and baby food; and other. The WWEIA food categories also consists of 47 subgroups. For example, for the milk and dairy main group, the subgroups were milk, flavored milk, cheese, dairy drinks and substitutes, and yogurt. For these analyses we focused on the 47 subgroups.

Using the relevant Food Patterns Equivalent Database [33] milk, cheese, and yogurt servings of non-dairy foods and especially mixed dishes were determined. The nutrient composition in the relevant Food and Nutrient Database for Dietary Studies FNDDS 2011-2012 and 2013-2014 [34] linked to SR 26 and SR 28 respectively [1] for milk, NFS (not further specified); cheese, NFS; and yogurt, NFS was used to assess energy and nutrient contribution of dairy servings non-dairy foods. The nutrients reported herein are the nutrients of public health concern [2]: dietary fiber, calcium, vitamin $\mathrm{D}$, and potassium; and nutrients to limit: SFA, added sugars, and sodium.

Data are reported as specific food group (SFG) intake, adjusted intake, and delta intake. Specific food group intake is intake from the dairy food groups (milk, cheese, and yogurt). Adjusted intake is the total daily intake after nutrients from dairy from non-dairy foods (e.g., mixed dishes) have been included, and reflect the disaggregation. Delta intake is the amount of nutrients from dairy in non-dairy foods that was added to or removed from the specific food group intake to calculate the adjusted intake. The consumer number $(n)$ for delta was the number of subjects that consumed dairy from mixed dishes.

\subsection{Statistical Analyses}

Data were analyzed using SAS 9.2 and SUDAAN release 11.0 (Research Triangle Institute, Research Triangle Park, NC, USA) with survey parameters including strata, primary sampling units, and dietary sample weights [26]. Means and standard errors (SE) of energy and nutrient intakes from the total diet 
and from each food group were determined using PROC DESCRIPT of SUDAAN. Percentages of total energy and nutrient intakes from each food group were calculated from the average consumption of each food. Mean intakes were tabulated by ranked order to $1 \%$ of consumption.

\section{Results}

\subsection{Contribution of Foods to Percent Energy Intake}

Total mean daily energy consumption was $1535 \pm 19$ kcals \pm SE; $1953 \pm 23.0$ kcals; and $2056.0 \pm 33.2$ kcals, respectively for children 2-5, 6-11, and 12-18 years, respectively. Table 1 shows the food sources contributing at least $1 \%$ of percent energy intake from the WWEIA sub-categories. There were 31, 29, and 31 food sources that contributed at least 1\% of SFG energy intake of children 2-5, 6-11, and 12-18 years, respectively. Using SFG intake data (kcals; \% of energy) for children 2-5 years, milk (136 kcals; $8.9 \%$ of energy), sweet bakery products (116 kcals; $7.6 \%$ ), and grain-based mixed dishes ( 86 kcals; $5.6 \%$ ) were ranked as the top food sources of energy. Cheese was ranked as the 18th food source of energy (39 kcals; 2.5\%). Using adjusted (disaggregated) data, milk and sweet bakery products remained the two top ranked foods (151 kcals; $9.8 \%$ and $115 \mathrm{kcals} ; 7.5 \%$ ) with mean delta intakes of +15 and -1 kcals, respectively. Cheese was the fifth ranked energy source (74 kcals; $4.8 \%)$.

The top SFG sources of energy for children 6-11 years were sweet bakery products (164 kcals; $8.4 \%$ ), pizza (132 kcals; 6.8\%), and sweetened beverages (112 kcals; 5.7\%), with milk and cheese ranked 7th (96 kcals; $4.9 \%$ ) and 21st (38 kcals; $1.9 \%)$, respectively. Using adjusted data, sweet bakery products and milk ranked first (163 kcals; $8.3 \%$ ) and second (117 kcals; 6.0\%), respectively, with mean delta intakes of -2 and +21 kcals.

For children 12-18 years, the top SFG intakes of food sources were sweetened beverages (162 kcals; $7.9 \%$ ), sweet bakery products (139 kcals; 6.8\%), and pizza (135 kcals; $6.6 \%$ ), with milk and cheese ranked 7 th (96 kcals; 4.7\%) and 18th (42 kcals; $2.1 \%$ ), respectively. Using adjusted data sweetened beverages and sweet bakery products continued to rank first (160 kcals; $7.8 \%$ ) and second (137 kcals; $6.7 \%)$; respectively; each with a delta value of -1 kcals. Milk (117 kcals; $5.7 \%)$ and cheese (118 kcals; $5.7 \%$ ) were the fourth and fifth ranked food groups, with mean delta intakes of +21.5 kcals and +74 kcals.

\subsection{Contribution of Foods to Percent Fiber Intake}

Total mean daily dietary fiber intake was $11.8 \pm 0.2 \mathrm{~g} ; 14.6 \pm 0.3 \mathrm{~g}$; and $14.7 \pm 0.3 \mathrm{~g}$, for children $2-5,6-11$, and 12-18 years, respectively. Table 2 shows the food sources contributing to at least $1 \%$ of daily fiber intake. In all three age groups there were 22 different food groups that contributed at least $1 \%$ of fiber intake. For the specific food group intakes, fruit was the top contributor to fiber intake with a mean of $2 \mathrm{~g} ; 17.2 \% ; 1.9 \mathrm{~g} ; 12.8 \%$, and $1.5 \mathrm{~g} ; 10 \%$, for each age group, respectively. For children $2-5$ years, SFG intake was followed by bread, rolls, tortillas $(1.2 \mathrm{~g} ; 10 \%)$ and ready-to-eat cereal (RTEC) (0.9 g; 7.4\%). For children 6-11 years, bread, rolls, tortillas $(1.5 \mathrm{~g} ; 10 \%)$ and mixed dishes-pizza $(1.1 \%$; $7.4 \%$ ) followed; finally, in children 12-18 years bread, rolls, tortillas (1.5 g; 10\%), was followed by mixed dishes-Mexican (1.1 g; 7.7\%). There were no differences in rank order after adjustment of any of the foods in all three age groups and delta intake was zero. 
Table 1. Food/food group sources of mean energy (kcals) intake ${ }^{1}$ among US children aged $2-18$ years $(N=5876)$ : National Health and Nutrition Examination Survey 2011-2014.

\begin{tabular}{|c|c|c|c|c|c|c|c|c|c|c|c|c|c|c|c|c|c|}
\hline \multicolumn{18}{|c|}{ Mean Energy Intake (kcals) of Children 2-5 Years of Age $(n=1511)$} \\
\hline \multirow{2}{*}{$\begin{array}{c}\text { WWEIA Food Group } \\
\text { Sub Group Description }\end{array}$} & \multicolumn{6}{|c|}{ Specific Food Group Intake } & \multicolumn{6}{|c|}{ Adjusted Intake $^{2}$} & \multicolumn{5}{|c|}{ Delta Intake } \\
\hline & Cons & Rank & Mean & SE & Pct & SE & Cons & Rank & Mean & SE & Pct & $\mathrm{SE}$ & Cons & Mean & SE & Pct & $\mathrm{SE}$ \\
\hline Milk & 1120 & 1 & 136 & 5.5 & 8.9 & 0.3 & 1415 & 1 & 151 & 5.3 & 9.8 & 0.3 & 1120 & 15 & 0.7 & 1.0 & 0.1 \\
\hline Sweet Bakery Products & 723 & 2 & 116 & 5.3 & 7.6 & 0.3 & 723 & 2 & 115 & 5.5 & 7.5 & 0.3 & 262 & -1 & 0.1 & 0 & 0 \\
\hline Mixed Dishes-Grain-based & 466 & 3 & 86 & 6.5 & 5.6 & 0.4 & 466 & 4 & 79 & 6.2 & 5.1 & 0.4 & 266 & -7 & 0.7 & -0.5 & 0 \\
\hline Breads, Rolls, Tortillas & 818 & 4 & 83 & 6.2 & 5.4 & 0.4 & 818 & 3 & 83 & 6.2 & 5.4 & 0.4 & 30 & 0 & 0.1 & 0 & 0 \\
\hline $100 \%$ Juice & 755 & 5 & 66 & 4.1 & 4.3 & 0.3 & 755 & 6 & 66 & 4.1 & 4.3 & 0.3 & 0 & 0 & 0.0 & 0 & 0 \\
\hline Savory Snacks & 688 & 6 & 65 & 6.6 & 4.2 & 0.4 & 688 & 7 & 65 & 6.5 & 4.2 & 0.4 & 140 & 0 & 0.0 & 0 & 0 \\
\hline Fruits & 945 & 7 & 63 & 2.3 & 4.1 & 0.2 & 945 & 8 & 63 & 2.3 & 4.1 & 0.2 & 2 & 0 & 0.0 & 0 & 0 \\
\hline Sweetened Beverages & 787 & 8 & 61 & 4.5 & 4.0 & 0.3 & 787 & 9 & 61 & 4.5 & 4.0 & 0.3 & 23 & 0 & 0.2 & 0 & 0 \\
\hline Poultry & 526 & 9 & 59 & 5.1 & 3.8 & 0.3 & 526 & 10 & 59 & 5.1 & 3.8 & 0.3 & 71 & 0 & 0.0 & 0 & 0 \\
\hline Mixed Dishes-Mexican & 201 & 10 & 54 & 6.2 & 3.5 & 0.4 & 201 & 13 & 43 & 4.9 & 2.8 & 0.3 & 189 & -11.3 & 1.4 & -0.7 & 0.1 \\
\hline Mixed Dishes_Pizza & 247 & 11 & 53 & 5.9 & 3.5 & 0.4 & 247 & 17 & 39 & 4.2 & 2.5 & 0.3 & 247 & -14 & 1.8 & -0.9 & 0.1 \\
\hline Ready-to-Eat Cereals & 695 & 12 & 51 & 2.6 & 3.3 & 0.2 & 695 & 11 & 51 & 2.6 & 3.3 & 0.2 & 12 & 0 & 0.0 & 0 & 0 \\
\hline Flavored Milk & 253 & 13 & 48 & 6.5 & 3.1 & 0.4 & 253 & 12 & 48 & 6.5 & 3.1 & 0.4 & 0 & 0 & 0.0 & 0 & 0 \\
\hline Quick Breads/Bread Products & 293 & 14 & 41 & 5.3 & 2.7 & 0.4 & 293 & 15 & 40 & 5.0 & 2.6 & 0.4 & 274 & -2 & 0.3 & -0.1 & 0 \\
\hline Cured Meats/Poultry & 459 & 15 & 40 & 4.0 & 2.6 & 0.3 & 459 & 14 & 40 & 4.0 & 2.6 & 0.3 & 2 & 0 & 0.1 & 0 & 0 \\
\hline Crackers & 353 & 16 & 40 & 3.9 & 2.6 & 0.3 & 353 & 16 & 39 & 3.9 & 2.6 & 0.3 & 86 & 0 & 0.1 & 0 & 0 \\
\hline Mixed Dishes-Sandwiches & 208 & 17 & 39 & 4.0 & 2.6 & 0.3 & 208 & 18 & 37 & 3.9 & 2.4 & 0.2 & 67 & -2 & 0.4 & 0 & 0 \\
\hline Cheese & 542 & 18 & 39 & 5.3 & 2.5 & 0.3 & 1047 & 5 & 74 & 5.7 & 4.8 & 0.4 & 820 & 36 & 1.8 & 2.3 & 0.1 \\
\hline Candy & 507 & 19 & 37 & 2.7 & 2.4 & 0.2 & 507 & 19 & 35 & 2.6 & 2.3 & 0.2 & 181 & -1 & 0.2 & -0.1 & 0 \\
\hline Other Desserts & 416 & 20 & 37 & 3.6 & 2.4 & 0.2 & 416 & 21 & 32 & 3.2 & 2.1 & 0.2 & 291 & -5 & 0.6 & -0.3 & 0 \\
\hline White Potatoes & 401 & 21 & 35 & 3.7 & 2.3 & 0.2 & 401 & 20 & 34 & 3.7 & 2.2 & 0.2 & 78 & 0 & 0.2 & 0 & 0 \\
\hline Plant-Based Protein Foods & 346 & 22 & 30 & 2.5 & 2.0 & 0.2 & 346 & 22 & 30 & 2.5 & 2.0 & 0.2 & 1 & 0 & 0.0 & 0 & 0 \\
\hline Yogurt & 231 & 23 & 27 & 3.1 & 1.8 & 0.2 & 278 & 23 & 28 & 3.1 & 1.9 & 0.2 & 60 & 1 & 0.4 & 0.1 & 0 \\
\hline Eggs & 332 & 24 & 27 & 2.6 & 1.7 & 0.2 & 332 & 25 & 24 & 2.4 & 1.6 & 0.2 & 234 & -3 & 0.5 & -0.2 & 0 \\
\hline Cooked Grains & 299 & 25 & 26 & 2.8 & 1.7 & 0.2 & 299 & 24 & 26 & 2.8 & 1.7 & 0.2 & 0 & 0 & 0.0 & 0 & 0 \\
\hline Mixed Dishes-M/P/F & 189 & 26 & 22 & 2.9 & 1.4 & 0.2 & 189 & 26 & 21 & 2.8 & 1.4 & 0.2 & 69 & -1 & 0.2 & 0 & 0 \\
\hline Vegetables, excluding Potatoes & 560 & 27 & 19 & 1.9 & 1.2 & 0.1 & 560 & 27 & 18 & 1.9 & 1.2 & 0.1 & 22 & 0 & 0.2 & 0 & 0 \\
\hline Fats and Oils & 460 & 28 & 17 & 1.3 & 1.1 & 0.1 & 460 & 28 & 17 & 1.3 & 1.1 & 0.1 & 31 & 0 & 0.0 & 0 & 0 \\
\hline Mixed Dishes-Soups & 227 & 29 & 16 & 2.5 & 1.0 & 0.2 & 227 & 29 & 16 & 2.4 & 1.0 & 0.2 & 7 & 0 & 0.1 & 0 & 0 \\
\hline Sugars & 440 & 30 & 15 & 2.2 & 1.0 & 0.1 & 440 & 30 & 16 & 2.2 & 1.0 & 0.1 & 21 & 0 & 0.0 & 0 & 0 \\
\hline Meats & 220 & 31 & 16 & 2.1 & 1.0 & 0.1 & 220 & 31 & 16 & 2.1 & 1.0 & 0.1 & 5 & 0 & 0.0 & 0 & 0 \\
\hline
\end{tabular}


Table 1. Cont.

\begin{tabular}{|c|c|c|c|c|c|c|c|c|c|c|c|c|c|c|c|c|c|}
\hline \multicolumn{18}{|c|}{ Mean Energy Intake (kcals) in Children 6-11 Years of Age $(n=2193)$} \\
\hline \multirow{2}{*}{$\begin{array}{l}\text { WWEIA Food Group } \\
\text { Sub Group Description }\end{array}$} & \multicolumn{6}{|c|}{ Specific Food Group Intake } & \multicolumn{6}{|c|}{ Adjusted Intake } & \multicolumn{5}{|c|}{ Delta Intake } \\
\hline & Cons & Rank & Mean & SE & Pct & SE & Cons & Rank & Mean & SE & Pct & SE & Cons & Mean & SE & Pct & SE \\
\hline Sweet Bakery Products & 1034 & 1 & 164 & 9.1 & 8.4 & 0.4 & 1034 & 1 & 163 & 8.9 & 8.3 & 0.4 & 455 & -2 & 0.2 & -0.1 & 0.01 \\
\hline Mixed Dishes—Pizza & 548 & 2 & 132 & 14.6 & 6.8 & 0.7 & 548 & 6 & 100 & 11.1 & 5.1 & 0.6 & 547 & -32 & 3.6 & -1.7 & 0.2 \\
\hline Sweetened Beverages & 1467 & 3 & 112 & 4.1 & 5.7 & 0.2 & 1467 & 3 & 110 & 4.1 & 5.7 & 0.2 & 45 & -1 & 0.7 & -0.1 & 0.04 \\
\hline Breads, Rolls, Tortillas & 1205 & 4 & 109 & 3.6 & 5.6 & 0.2 & 1205 & 4 & 109 & 3.5 & 5.6 & 0.2 & 85 & 0 & 0.1 & -0.02 & 0.00 \\
\hline Mixed Dishes-Grain-based & 606 & 5 & 105 & 7.8 & 5.4 & 0.4 & 606 & 7 & 95 & 6.6 & 4.9 & 0.3 & 324 & -10 & 1.5 & -0.5 & 0.1 \\
\hline Mixed Dishes-Mexican & 362 & 6 & 99 & 11.0 & 5.1 & 0.5 & 362 & 9 & 80 & 8.8 & 4.1 & 0.4 & 347 & -19 & 2.4 & -1.0 & 0.1 \\
\hline Milk & 1274 & 7 & 96 & 3.3 & 4.9 & 0.2 & 1987 & 2 & 117 & 3.4 & 6.0 & 0.2 & 1646 & 21 & 1.0 & 1.0 & 0.1 \\
\hline Savory Snacks & 1048 & 8 & 89 & 4.8 & 4.6 & 0.2 & 1048 & 8 & 89 & 4.8 & 4.5 & 0.2 & 232 & 0 & 0.04 & -0.01 & 0.00 \\
\hline Mixed Dishes-Sandwiches & 436 & 9 & 79 & 7.0 & 4.1 & 0.3 & 436 & 11 & 74 & 6.6 & 3.8 & 0.3 & 170 & -5 & 0.8 & -0.3 & 0.04 \\
\hline Poultry & 711 & 10 & 77 & 5.5 & 4.0 & 0.3 & 711 & 10 & 77 & 5.5 & 3.9 & 0.3 & 124 & 0 & 0.03 & 0.00 & 0.00 \\
\hline Other Desserts & 620 & 11 & 65 & 7.2 & 3.4 & 0.4 & 620 & 15 & 56 & 6.6 & 2.9 & 0.3 & 478 & -10 & 1.0 & -0.5 & 0.1 \\
\hline Ready-to-Eat Cereals & 861 & 12 & 61 & 3.7 & 3.1 & 0.2 & 861 & 12 & 61 & 3.7 & 3.1 & 0.2 & 24 & 0 & 0.03 & 0.00 & 0.00 \\
\hline Quick Breads/Bread Products & 475 & 13 & 59 & 4.0 & 3.0 & 0.2 & 475 & 14 & 57 & 3.9 & 2.9 & 0.2 & 445 & -3 & 0.2 & -0.1 & 0.01 \\
\hline Candy & 764 & 14 & 58 & 7.4 & 3.0 & 0.4 & 764 & 13 & 57 & 7.3 & 2.9 & 0.4 & 306 & -1 & 0.2 & -0.1 & 0.01 \\
\hline Flavored Milk & 555 & 15 & 55 & 5.0 & 2.8 & 0.3 & 555 & 16 & 55 & 5.0 & 2.8 & 0.3 & 0 & 0 & 0.00 & 0.00 & 0.00 \\
\hline Fruits & 1153 & 16 & 54 & 2.4 & 2.8 & 0.1 & 1153 & 17 & 54 & 2.4 & 2.8 & 0.1 & 6 & 0 & 0.01 & 0.00 & 0.00 \\
\hline White Potatoes & 610 & 17 & 46 & 3.3 & 2.3 & 0.2 & 610 & 18 & 44 & 3.2 & 2.3 & 0.2 & 105 & -1 & 0.3 & -0.1 & 0.02 \\
\hline Mixed Dishes-M/F/P & 281 & 18 & 44 & 7.4 & 2.3 & 0.4 & 281 & 19 & 43 & 7.2 & 2.2 & 0.4 & 123 & -1 & 0.4 & -0.1 & 0.02 \\
\hline $100 \%$ Juice & 759 & 19 & 41 & 3.2 & 2.1 & 0.2 & 759 & 20 & 41 & 3.2 & 2.1 & 0.2 & 0 & 0 & 0.00 & 0.00 & 0.00 \\
\hline Cured Meats/Poultry & 687 & 20 & 38 & 2.7 & 1.9 & 0.1 & 687 & 21 & 38 & 2.7 & 1.9 & 0.1 & 1 & 0 & 0.00 & 0.00 & 0.00 \\
\hline Cheese & 723 & 21 & 38 & 3.0 & 1.9 & 0.2 & 1643 & 5 & 106 & 4.4 & 5.4 & 0.2 & 1349 & 69 & 4.8 & 3.5 & 0.2 \\
\hline Plant-Based Protein Foods & 475 & 22 & 35 & 3.8 & 1.8 & 0.2 & 475 & 22 & 35 & 3.8 & 1.8 & 0.2 & 1 & 0 & 0.00 & 0.00 & 0.00 \\
\hline Meats & 388 & 23 & 31 & 3.3 & 1.6 & 0.2 & 388 & 23 & 31 & 3.3 & 1.6 & 0.2 & 5 & 0 & 0.01 & 0.00 & 0.00 \\
\hline Fats and Oils & 740 & 24 & 29 & 2.2 & 1.5 & 0.1 & 740 & 24 & 29 & 2.1 & 1.5 & 0.1 & 62 & 0 & 0.03 & 0.00 & 0.00 \\
\hline Cooked Grains & 302 & 25 & 27 & 3.1 & 1.4 & 0.2 & 302 & 25 & 27 & 3.1 & 1.4 & 0.2 & 1 & 0 & 0.1 & 0.00 & 0.00 \\
\hline Crackers & 325 & 26 & 25 & 2.5 & 1.3 & 0.1 & 325 & 26 & 25 & 2.5 & 1.3 & 0.1 & 96 & 0 & 0.1 & -0.01 & 0.00 \\
\hline Eggs & 324 & 27 & 23 & 2.2 & 1.2 & 0.1 & 324 & 28 & 21 & 2.0 & 1.1 & 0.1 & 217 & -2 & 0.4 & -0.1 & 0.02 \\
\hline Sugars & 609 & 28 & 21 & 1.8 & 1.1 & 0.1 & 609 & 27 & 21 & 1.8 & 1.1 & 0.1 & 42 & 0 & 0.05 & -0.01 & 0.00 \\
\hline Mixed Dishes-Soups & 253 & 29 & 20 & 2.3 & 1.0 & 0.1 & 253 & 29 & 19 & 2.1 & 1.0 & 0.1 & 6 & -1 & 0.5 & -0.03 & 0.02 \\
\hline
\end{tabular}


Table 1. Cont.

\begin{tabular}{|c|c|c|c|c|c|c|c|c|c|c|c|c|c|c|c|c|c|}
\hline \multicolumn{18}{|c|}{ Mean Energy Intake (kcals) $12-18$ Years of Age $(n=2172)$} \\
\hline \multirow{2}{*}{$\begin{array}{c}\text { WWEIA Food Group } \\
\text { Sub Group Description }\end{array}$} & \multicolumn{6}{|c|}{ Specific Food Group Intake } & \multicolumn{6}{|c|}{ Adjusted Intake } & \multicolumn{5}{|c|}{ Delta Intake } \\
\hline & Cons & Rank & Mean & SE & Pct & SE & Cons & Rank & Mean & SE & Pct & SE & Cons & Mean & SE & Pct & SE \\
\hline Sweetened Beverages & 1404 & 1 & 162 & 6.0 & 7.9 & 0.3 & 1404 & 1 & 160 & 5.9 & 7.8 & 0.3 & 40 & -1 & 0.4 & -0.1 & 0.02 \\
\hline Sweet Bakery Products & 833 & 2 & 139 & 10.2 & 6.8 & 0.5 & 833 & 2 & 137 & 10.1 & 6.7 & 0.5 & 344 & -1 & 0.2 & -0.1 & 0.01 \\
\hline Mixed Dishes_-Pizza & 486 & 3 & 135 & 14.2 & 6.6 & 0.7 & 486 & 6 & 103 & 10.8 & 5.0 & 0.5 & 486 & -33 & 3.6 & -1.6 & 0.2 \\
\hline Breads, Rolls, Tortillas & 1119 & 4 & 118 & 5.2 & 5.7 & 0.2 & 1119 & 3 & 118 & 5.2 & 5.7 & 0.2 & 85 & 0.0 & 0.1 & -0.02 & 0.0 \\
\hline Mixed Dishes-Grain-based & 464 & 5 & 105 & 8.0 & 5.1 & 0.4 & 464 & 7 & 96 & 7.6 & 4.7 & 0.4 & 244 & -10 & 1.3 & -0.5 & 0.1 \\
\hline Mixed Dishes-Mexican & 329 & 6 & 103 & 11.3 & 5.0 & 0.6 & 329 & 10 & 84 & 9.0 & 4.1 & 0.4 & 313 & -19 & 2.6 & -0.9 & 0.1 \\
\hline Milk & 973 & 7 & 96 & 4.8 & 4.7 & 0.2 & 1779 & 4 & 117 & 4.7 & 5.7 & 0.2 & 1453 & 21.5 & 1.1 & 1.0 & 0.1 \\
\hline Savory Snacks & 942 & 8 & 93 & 6.1 & 4.5 & 0.3 & 942 & 8 & 92 & 6.1 & 4.0 & 0.3 & 233 & 0.0 & 0.1 & -0.02 & 0.0 \\
\hline Mixed Dishes-Sandwiches & 374 & 9 & 91 & 8.6 & 4.4 & 0.4 & 374 & 11 & 83 & 7.6 & 4.0 & 0.4 & 174 & -8 & 1.4 & -0.4 & 0.1 \\
\hline Poultry & 652 & 10 & 87 & 8.1 & 4.2 & 0.4 & 652 & 9 & 87 & 8.1 & 4.2 & 0.4 & 130 & 0.0 & 0.02 & -0.01 & 0.0 \\
\hline White Potatoes & 580 & 11 & 63 & 4.3 & 3.1 & 0.2 & 580 & 12 & 60 & 4.0 & 2.9 & 0.2 & 121 & -4 & 0.7 & -0.2 & 0.03 \\
\hline Ready-to-Eat Cereals & 582 & 12 & 57 & 4.0 & 2.8 & 0.2 & 582 & 13 & 57 & 4.0 & 2.8 & 0.2 & 18 & 0.0 & 0.01 & 0.0 & 0.0 \\
\hline Meats & 424 & 13 & 54 & 4.6 & 2.6 & 0.2 & 424 & 14 & 54 & 4.6 & 2.6 & 0.2 & 14 & 0.0 & 0.01 & 0.0 & 0.0 \\
\hline Mixed Dishes-M/P/F & 288 & 14 & 49 & 4.9 & 2.4 & 0.2 & 288 & 15 & 46 & 4.8 & 2.2 & 0.2 & 100 & -2 & 0.6 & -0.1 & 0.03 \\
\hline Other Desserts & 377 & 15 & 48 & 5.8 & 2.3 & 0.3 & 377 & 22 & 39 & 4.0 & 1.9 & 0.2 & 339 & -9 & 2.1 & -0.5 & 0.1 \\
\hline Quick Breads/Bread Products & 297 & 16 & 44 & 4.1 & 2.1 & 0.2 & 297 & 17 & 42 & 4.0 & 2.0 & 0.2 & 282 & -2 & 0.2 & -0.1 & 0.01 \\
\hline Candy & 600 & 17 & 43 & 5.0 & 2.1 & 0.2 & 600 & 18 & 42 & 4.9 & 2.0 & 0.2 & 273 & -1 & 0.2 & -0.1 & 0.01 \\
\hline Cheese & 687 & 18 & 42 & 3.1 & 2.1 & 0.2 & 1565 & 5 & 118 & 5.2 & 5.7 & 0.3 & 1276 & 74 & 4.9 & 3.6 & 0.2 \\
\hline Fruits & 834 & 19 & 42 & 3.1 & 2.1 & 0.2 & 834 & 16 & 42 & 3.1 & 2.1 & 0.2 & 3 & 0.0 & 0.01 & 0.0 & 0.0 \\
\hline Cured Meats/Poultry & 585 & 20 & 42 & 4.1 & 2.0 & 0.2 & 585 & 19 & 41 & 4.1 & 2.0 & 0.2 & 2 & 0.0 & 0.2 & -0.01 & 0.01 \\
\hline Mixed Dishes-Asian & 174 & 21 & 41 & 8.4 & 2.0 & 0.4 & 174 & 20 & 41 & 8.4 & 2.0 & 0.4 & 11 & 0.0 & 0.0 & 0.0 & 0.0 \\
\hline Plant-Based Protein Foods & 344 & 22 & 41 & 6.1 & 2.0 & 0.3 & 344 & 21 & 41 & 6.1 & 2.0 & 0.3 & 1 & 0.00 & 0.0 & 0.0 & 0.0 \\
\hline Fats and Oils & 700 & 23 & 38 & 3.6 & 1.9 & 0.2 & 700 & 23 & 38 & 3.5 & 1.9 & 0.2 & 86 & 0.0 & 0.04 & -0.01 & 0.0 \\
\hline $100 \%$ Juice & 487 & 24 & 36 & 4.4 & 1.8 & 0.2 & 487 & 24 & 36 & 4.4 & 1.8 & 0.2 & 0 & 0.0 & 0.0 & 0.0 & 0.0 \\
\hline Coffee and Tea & 505 & 25 & 36 & 5.8 & 1.8 & 0.3 & 505 & 25 & 34 & 5.9 & 1.6 & 0.3 & 60 & -3 & 0.4 & -0.1 & 0.02 \\
\hline Cooked Grains & 325 & 26 & 31 & 3.0 & 1.5 & 0.2 & 325 & 26 & 31 & 3.0 & 1.5 & 0.2 & 0 & 0.0 & 0.0 & 0.0 & 0.0 \\
\hline Eggs & 333 & 27 & 28 & 2.3 & 1.3 & 0.1 & 333 & 28 & 25 & 2.1 & 1.2 & 0.1 & 211 & -2 & 0.3 & -0.1 & 0.01 \\
\hline Flavored Milk & 225 & 28 & 25 & 3.0 & 1.2 & 0.1 & 225 & 27 & 25 & 3.0 & 1.2 & 0.1 & 0 & 0.0 & 0.0 & 0.0 & 0.0 \\
\hline Crackers & 229 & 29 & 25 & 3.7 & 1.2 & 0.2 & 229 & 29 & 25 & 4.0 & 1.2 & 0.2 & 69 & 0.0 & 0.1 & -0.01 & 0.0 \\
\hline Mixed Dishes-Soups & 237 & 30 & 21 & 2.0 & 1.0 & 0.1 & 237 & 31 & 21 & 1.9 & 1.0 & 0.1 & 10 & -1 & 0.2 & -0.03 & 0.01 \\
\hline Sugars & 446 & 31 & 21 & 3.3 & 1.0 & 0.2 & 446 & 30 & 21 & 3.3 & 1.0 & 0.2 & 16 & 0.0 & 0.01 & 0.0 & 0.0 \\
\hline
\end{tabular}

${ }^{1}$ To a $1 \%$ contribution of daily intake of energy; ${ }^{2}$ Nutrients from milk, cheese, and yogurt for non-dairy foods are added to the nutrients in the milk, cheese, and yogurt food categories, respectively. For non-dairy foods the nutrients displayed are only for the milk, cheese, and yogurt in the non-dairy food. Abbreviations: Cons $=$ consumers, $\mathrm{M} / \mathrm{P} / \mathrm{F}=\mathrm{meat} / \mathrm{poultry} /$ fish $\mathrm{SE}=$ standard error; $\mathrm{Pct}=$ percent contribution to energy intake or specific nutrient intake, as appropriate. 
Table 2. Food/food group sources ${ }^{1}$ of mean dietary fiber $(\mathrm{g})$ intake among US children aged 2-18 years $(N=5876)$ : National Health and Nutrition Examination Survey 2011-2014.

\begin{tabular}{|c|c|c|c|c|c|c|c|c|c|c|c|c|c|c|c|c|c|}
\hline \multicolumn{18}{|c|}{ Mean Dietary Fiber Intake (g) of Children 2-5 Years of Age $(n=1511)$} \\
\hline \multirow[b]{2}{*}{ Sub Group Description } & \multicolumn{6}{|c|}{ Specific Food Group Intake } & \multicolumn{6}{|c|}{ Adjusted Intake ${ }^{2}$} & \multicolumn{5}{|c|}{ Delta Intake } \\
\hline & Cons & Rank & Mean & SE & Pct & SE & Cons & Rank & Mean & SE & Pct & SE & Cons & Mean & SE & Pct & SE \\
\hline Fruit & 945 & 1 & 2.0 & 0.1 & 17.2 & 0.4 & 945 & 1 & 2.0 & 0.1 & 17.2 & 0.4 & 2 & 0.0 & 0.0 & 0.0 & 0.0 \\
\hline Breads, Rolls, Tortillas & 818 & 2 & 1.2 & 0.1 & 10.0 & 0.7 & 818 & 2 & 1.2 & 0.1 & 10.0 & 0.7 & 30 & 0.0 & 0.0 & 0.0 & 0.0 \\
\hline Ready-to-Eat Cereals & 695 & 3 & 0.9 & 0.1 & 7.4 & 0.5 & 695 & 3 & 0.9 & 0.1 & 7.4 & 0.5 & 12 & 0.0 & 0.0 & 0.0 & 0.0 \\
\hline Vegetables, excluding Potatoes & 560 & 4 & 0.9 & 0.1 & 7.3 & 0.5 & 560 & 4 & 0.9 & 0.1 & 7.3 & 0.5 & 22 & 0.0 & 0.0 & 0.0 & 0.0 \\
\hline Mixed Dishes_-Grain based & 466 & 5 & 0.8 & 0.1 & 7.0 & 0.7 & 466 & 5 & 0.8 & 0.1 & 7.0 & 0.7 & 266 & 0.0 & 0.0 & 0.0 & 0.0 \\
\hline Plant-Based Protein Foods & 346 & 6 & 0.6 & 0.1 & 5.2 & 0.5 & 346 & 6 & 0.6 & 0.1 & 5.2 & 0.5 & 1 & 0.0 & 0.0 & 0.0 & 0.0 \\
\hline Savory Snacks & 688 & 7 & 0.6 & 0.0 & 4.9 & 0.4 & 688 & 7 & 0.6 & 0.0 & 4.9 & 0.4 & 140 & 0.0 & 0.0 & 0.0 & 0.0 \\
\hline Sweet Bakery Products & 723 & 8 & 0.5 & 0.0 & 4.4 & 0.2 & 723 & 8 & 0.5 & 0.0 & 4.4 & 0.2 & 262 & 0.0 & 0.0 & 0.0 & 0.0 \\
\hline Mixed Dishes-Mexican & 201 & 9 & 0.4 & 0.1 & 4.0 & 0.5 & 201 & 9 & 0.5 & 0.1 & 4.0 & 0.5 & 189 & 0.0 & 0.0 & 0.0 & 0.0 \\
\hline Mixed Dishes-Pizza & 247 & 10 & 0.5 & 0.1 & 3.9 & 0.4 & 247 & 10 & 0.5 & 0.1 & 3.9 & 0.4 & 247 & 0.0 & 0.0 & 0.0 & 0.0 \\
\hline White Potatoes & 401 & 11 & 0.5 & 0.1 & 3.8 & 0.4 & 401 & 11 & 0.5 & 0.1 & 3.8 & 0.4 & 78 & 0.0 & 0.0 & 0.0 & 0.0 \\
\hline $100 \%$ Juice & 755 & 12 & 0.3 & 0.0 & 2.6 & 0.2 & 755 & 12 & 0.3 & 0.0 & 2.6 & 0.2 & 0 & 0.0 & 0.0 & 0.0 & 0.0 \\
\hline Quick Breads and Bread Products & 293 & 13 & 0.3 & 0.1 & 2.5 & 0.6 & 293 & 13 & 0.3 & 0.1 & 2.5 & 0.6 & 274 & 0.0 & 0.0 & 0.0 & 0.0 \\
\hline Flavored Milk & 253 & 14 & 0.3 & 0.0 & 2.2 & 0.4 & 253 & 14 & 0.3 & 0.0 & 2.2 & 0.4 & 0 & 0.0 & 0.0 & 0.0 & 0.0 \\
\hline Crackers & 353 & 15 & 0.2 & 0.0 & 1.9 & 0.2 & 353 & 15 & 0.2 & 0.0 & 1.9 & 0.2 & 86 & 0.0 & 0.0 & 0.0 & 0.0 \\
\hline Poultry & 526 & 16 & 0.2 & 0.0 & 1.5 & 0.3 & 526 & 16 & 0.2 & 0.0 & 1.5 & 0.3 & 71 & 0.0 & 0.0 & 0.0 & 0.0 \\
\hline Cooked Cereal & 125 & 17 & 0.2 & 0.0 & 1.5 & 0.2 & 125 & 17 & 0.2 & 0.0 & 1.5 & 0.2 & 69 & 0.0 & 0.0 & 0.0 & 0.0 \\
\hline Mixed Dishes-Sandwiches & 208 & 18 & 0.2 & 0.0 & 1.5 & 0.2 & 208 & 18 & 0.2 & 0.0 & 1.5 & 0.2 & 67 & 0.0 & 0.0 & 0.0 & 0.0 \\
\hline Mixed Dishes-Soups & 227 & 19 & 0.2 & 0.0 & 1.5 & 0.3 & 227 & 19 & 0.2 & 0.0 & 1.45 & 0.26 & 7 & 0.0 & 0.0 & 0.0 & 0.0 \\
\hline Mixed Dishes-M/P/F & 189 & 20 & 0.2 & 0.0 & 1.4 & 0.2 & 189 & 20 & 0.2 & 0.0 & 1.37 & 0.22 & 69 & 0.0 & 0.0 & 0.0 & 0.0 \\
\hline Cooked Grains & 299 & 21 & 0.2 & 0.0 & 1.4 & 0.2 & 299 & 21 & 0.2 & 0.0 & 1.35 & 0.23 & 0 & 0.0 & 0.0 & 0.0 & 0.0 \\
\hline Snack/Meal Bars & 81 & 22 & 0.2 & 0.0 & 1.3 & 0.2 & 81 & 22 & 0.2 & 0.0 & 1.25 & 0.19 & 30 & 0.0 & 0.0 & 0.0 & 0.0 \\
\hline
\end{tabular}


Table 2. Cont

\begin{tabular}{|c|c|c|c|c|c|c|c|c|c|c|c|c|c|c|c|c|c|}
\hline \multicolumn{18}{|c|}{ Mean Dietary Fiber Intake (g) of Children 6-11 Years of Age $(n=2193)$} \\
\hline \multirow[b]{2}{*}{ Sub Group Description } & \multicolumn{6}{|c|}{ Specific Food Group Intake } & \multicolumn{6}{|c|}{ Adjusted Intake } & \multicolumn{5}{|c|}{ Delta Intake } \\
\hline & Cons & Rank & Mean & SE & Pct & SE & Cons & Rank & Mean & SE & Pct & SE & Cons & Mean & SE & Pct & SE \\
\hline Fruit & 1153 & 1 & 1.9 & 0.1 & 12.8 & 0.4 & 1153 & 1 & 1.9 & 0.1 & 12.8 & 0.5 & 6 & 0.0 & 0.0 & 0.0 & 0.0 \\
\hline Breads, Rolls, Tortillas & 1205 & 2 & 1.5 & 0.1 & 10.0 & 0.4 & 1205 & 2 & 1.5 & 0.1 & 10.0 & 0.4 & 85 & 0.0 & 0.0 & 0.0 & 0.0 \\
\hline Mixed Dishes—Pizza & 548 & 3 & 1.1 & 0.1 & 7.4 & 0.6 & 548 & 3 & 1.1 & 0.1 & 7.4 & 0.6 & 547 & 0.0 & 0.0 & 0.0 & 0.0 \\
\hline Mixed Dishes-Mexican & 362 & 4 & 1.0 & 0.1 & 6.8 & 0.7 & 362 & 4 & 1.0 & 0.1 & 6.8 & 0.7 & 347 & 0.0 & 0.0 & 0.0 & 0.0 \\
\hline Ready-to-Eat Cereals & 861 & 5 & 1.0 & 0.1 & 6.7 & 0.4 & 861 & 5 & 1.0 & 0.1 & 6.7 & 0.4 & 24 & 0.0 & 0.0 & 0.0 & 0.0 \\
\hline Mixed Dishes-Grain based & 606 & 6 & 1.0 & 0.1 & 6.5 & 0.4 & 606 & 6 & 1.0 & 0.1 & 6.5 & 0.4 & 324 & 0.0 & 0.0 & 0.0 & 0.0 \\
\hline Savory Snacks & 1048 & 7 & 0.9 & 0.0 & 6.1 & 0.3 & 1048 & 7 & 1.0 & 0.0 & 6.1 & 0.3 & 232 & 0.0 & 0.0 & 0.0 & 0.0 \\
\hline Vegetables, excluding Potatoes & 792 & 8 & 0.8 & 0.1 & 5.4 & 0.5 & 792 & 8 & 0.8 & 0.1 & 5.4 & 0.5 & 40 & 0.0 & 0.0 & 0.0 & 0.0 \\
\hline Plant-Based Protein Foods & 475 & 9 & 0.7 & 0.1 & 5.0 & 0.5 & 475 & 9 & 0.7 & 0.1 & 5.0 & 0.5 & 1 & 0.0 & 0.0 & 0.0 & 0.0 \\
\hline Sweet Bakery Products & 1034 & 10 & 0.7 & 0.0 & 4.9 & 0.3 & 1034 & 10 & 0.7 & 0.0 & 4.9 & 0.3 & 455 & 0.0 & 0.0 & 0.0 & 0.0 \\
\hline White Potatoes & 610 & 11 & 0.6 & 0.0 & 3.9 & 0.3 & 610 & 11 & 0.6 & 0.0 & 3.9 & 0.3 & 105 & 0.0 & 0.0 & 0.0 & 0.0 \\
\hline Quick Breads and Bread Products & 475 & 12 & 0.4 & 0.0 & 2.5 & 0.2 & 475 & 12 & 0.4 & 0.0 & 2.5 & 0.2 & 445 & 0.0 & 0.0 & 0.0 & 0.0 \\
\hline Mixed Dishes_-Sandwiches & 436 & 13 & 0.3 & 0.0 & 2.3 & 0.2 & 436 & 13 & 0.3 & 0.0 & 2.3 & 0.2 & 170 & 0.0 & 0.0 & 0.0 & 0.0 \\
\hline Mixed Dishes- $\mathrm{M} / \mathrm{P} / \mathrm{F}$ & 281 & 14 & 0.3 & 0.1 & 2.2 & 0.5 & 281 & 14 & 0.3 & 0.1 & 2.2 & 0.5 & 123 & 0.0 & 0.0 & 0.0 & 0.0 \\
\hline Flavored Milk & 555 & 15 & 0.3 & 0.0 & 2.1 & 0.3 & 555 & 15 & 0.3 & 0.0 & 2.1 & 0.3 & 0 & 0.0 & 0.0 & 0.0 & 0.0 \\
\hline Poultry & 711 & 16 & 0.2 & 0.0 & 1.6 & 0.2 & 711 & 16 & 0.2 & 0.0 & 1.6 & 0.2 & 124 & 0.0 & 0.0 & 0.0 & 0.0 \\
\hline Other Desserts & 620 & 17 & 0.2 & 0.0 & 1.6 & 0.2 & 620 & 17 & 0.2 & 0.0 & 1.6 & 0.2 & 478 & 0.0 & 0.0 & 0.0 & 0.0 \\
\hline Snack/Meal Bars & 124 & 18 & 0.2 & 0.1 & 1.5 & 0.3 & 124 & 18 & 0.2 & 0.1 & 1.5 & 0.3 & 44 & 0.0 & 0.0 & 0.0 & 0.0 \\
\hline $100 \%$ Juice & 759 & 19 & 0.2 & 0.0 & 1.4 & 0.1 & 759 & 19 & 0.2 & 0.0 & 1.4 & 0.1 & 0 & 0.0 & 0.0 & 0.0 & 0.0 \\
\hline Mixed Dishes-Soups & 253 & 20 & 0.2 & 0.0 & 1.4 & 0.2 & 253 & 20 & 0.2 & 0.0 & 1.4 & 0.2 & 6 & 0.0 & 0.0 & 0.0 & 0.0 \\
\hline Crackers & 325 & 21 & 0.2 & 0.0 & 1.1 & 0.2 & 325 & 21 & 0.2 & 0.0 & 1.1 & 0.2 & 96 & 0.0 & 0.0 & 0.0 & 0.0 \\
\hline Cooked Grains & 302 & 22 & 0.2 & 0.0 & 1.0 & 0.1 & 302 & 22 & 0.2 & 0.0 & 1.0 & 0.1 & 1 & 0.0 & 0.0 & 0.0 & 0.0 \\
\hline
\end{tabular}


Table 2. Cont.

\begin{tabular}{|c|c|c|c|c|c|c|c|c|c|c|c|c|c|c|c|c|c|}
\hline \multirow[b]{3}{*}{ Sub Group Description } & \multicolumn{12}{|c|}{ Mean Dietary Fiber Intake (g) Children 12-18 Years of Age $(n=2172)$} & \multirow{2}{*}{\multicolumn{5}{|c|}{ Delta Intake }} \\
\hline & \multicolumn{6}{|c|}{ Specific Food Group Intake } & \multicolumn{6}{|c|}{ Adjusted Intake } & & & & & \\
\hline & Cons & Rank & Mean & SE & Pct & SE & Cons & Rank & Mean & SE & Pct & SE & Cons & Mean & SE & Pct & SE \\
\hline Fruit & 834 & 1 & 1.5 & 0.1 & 10.0 & 0.8 & 834 & 1 & 1.5 & 0.1 & 10.0 & 0.8 & 3 & 0.0 & 0.0 & 0.0 & 0.0 \\
\hline Bread, Rolls, Tortillas & 1119 & 2 & 1.5 & 0.1 & 10.0 & 0.6 & 1119 & 2 & 1.5 & 0.1 & 10.0 & 0.6 & 85 & 0.0 & 0.0 & 0.0 & 0.0 \\
\hline Mixed Dishes-Mexican & 329 & 3 & 1.1 & 0.2 & 7.7 & 1.0 & 329 & 3 & 1.1 & 0.2 & 7.7 & 1.0 & 313 & 0.0 & 0.0 & 0.0 & 0.0 \\
\hline Mixed Dishes-Pizza & 486 & 4 & 1.1 & 0.1 & 7.4 & 0.8 & 486 & 4 & 1.1 & 0.1 & 7.4 & 0.8 & 486 & 0.0 & 0.0 & 0.0 & 0.0 \\
\hline Mixed Dishes_-Grain based & 464 & 5 & 0.9 & 0.1 & 6.4 & 0.4 & 464 & 5 & 0.9 & 0.1 & 6.4 & 0.4 & 244 & 0.0 & 0.0 & 0.0 & 0.0 \\
\hline Vegetables, excluding Potatoes & 803 & 6 & 0.9 & 0.1 & 6.4 & 0.5 & 803 & 6 & 0.9 & 0.1 & 6.4 & 0.5 & 28 & 0.0 & 0.0 & 0.0 & 0.0 \\
\hline Savory Snacks & 942 & 7 & 0.9 & 0.1 & 6.3 & 0.5 & 942 & 7 & 0.9 & 0.1 & 6.3 & 0.5 & 233 & 0.0 & 0.0 & 0.0 & 0.0 \\
\hline Ready-to-Eat Cereals & 582 & 8 & 0.9 & 0.1 & 6.2 & 0.6 & 582 & 8 & 0.9 & 0.1 & 6.2 & 0.6 & 18 & 0.0 & 0.0 & 0.0 & 0.0 \\
\hline Plant-Based Protein Foods & 344 & 9 & 0.8 & 0.1 & 5.7 & 0.8 & 344 & 9 & 0.8 & 0.1 & 5.7 & 0.8 & 1 & 0.0 & 0.0 & 0.0 & 0.0 \\
\hline White Potatoes & 580 & 10 & 0.8 & 0.1 & 5.5 & 0.4 & 580 & 10 & 0.8 & 0.1 & 5.5 & 0.4 & 121 & 0.0 & 0.0 & 0.0 & 0.0 \\
\hline Sweet Bakery Products & 833 & 11 & 0.6 & 0.1 & 4.3 & 0.3 & 833 & 11 & 0.6 & 0.1 & 4.3 & 0.3 & 344 & 0.0 & 0.0 & 0.0 & 0.0 \\
\hline Mixed Dishes-Sandwiches & 374 & 12 & 0.4 & 0.0 & 2.8 & 0.3 & 374 & 12 & 0.4 & 0.0 & 2.8 & 0.3 & 174 & 0.0 & 0.0 & 0.0 & 0.0 \\
\hline Mixed Dishes-M/P/F & 288 & 13 & 0.3 & 0.0 & 2.2 & 0.2 & 288 & 13 & 0.3 & 0.0 & 2.2 & 0.2 & 100 & 0.0 & 0.0 & 0.0 & 0.0 \\
\hline Condiments and Sauces & 889 & 14 & 0.28 & 0.03 & 1.87 & 0.20 & 889 & 14 & 0.28 & 0.03 & 1.9 & 0.2 & 41 & 0.0 & 0.0 & 0.0 & 0.0 \\
\hline Quick Breads and Bread Products & 297 & 15 & 0.3 & 0.0 & 1.9 & 0.2 & 297 & 15 & 0.3 & 0.02 & 1.9 & 0.2 & 282 & 0.0 & 0.0 & 0.0 & 0.0 \\
\hline Poultry & 652 & 16 & 0.2 & 0.0 & 1.6 & 0.2 & 652 & 16 & 0.2 & 0.03 & 1.6 & 0.2 & 130 & 0.0 & 0.0 & 0.0 & 0.0 \\
\hline Snack/Meal Bars & 135 & 17 & 0.2 & 0.0 & 1.6 & 0.3 & 135 & 17 & 0.2 & 0.04 & 1.6 & 0.3 & 47 & 0.0 & 0.0 & 0.0 & 0.0 \\
\hline Mixed Dishes-Soups & 237 & 18 & 0.2 & 0.0 & 1.5 & 0.2 & 237 & 18 & 0.2 & 0.03 & 1.5 & 0.2 & 10 & 0.0 & 0.0 & 0.0 & 0.0 \\
\hline Cooked Grains & 325 & 19 & 0.2 & 0.0 & 1.3 & 0.1 & 325 & 19 & 0.2 & 0.02 & 1.3 & 0.1 & 0 & 0.0 & 0.0 & 0.0 & 0.0 \\
\hline $100 \%$ Juice & 487 & 20 & 0.2 & 0.0 & 1.3 & 0.1 & 487 & 20 & 0.2 & 0.02 & 1.3 & 0.1 & 0 & 0.0 & 0.0 & 0.0 & 0.0 \\
\hline Mixed Dishes-Asian & 174 & 21 & 0.2 & 0.0 & 1.2 & 0.2 & 174 & 21 & 0.2 & 0.03 & 1.2 & 0.2 & 11 & 0.0 & 0.0 & 0.0 & 0.0 \\
\hline Crackers & 229 & 22 & 0.2 & 0.0 & 1.0 & 0.2 & 229 & 22 & 0.2 & 0.02 & 1.0 & 0.2 & 69 & 0.0 & 0.0 & 0.0 & 0.0 \\
\hline
\end{tabular}

${ }^{1}$ To a $1 \%$ contribution of daily intake of dietary fiber; ${ }^{2}$ Nutrients from milk, cheese, and yogurt for non-dairy foods are added to the nutrients in the milk, cheese, and yogurt food categories, respectively. For non-dairy foods the nutrients displayed are only for the milk, cheese, and yogurt in the non-dairy food. Abbreviations: Cons $=$ consumers, $\mathrm{M} / \mathrm{P} / \mathrm{F}=$ meat / poultry / fish; SE = standard error; $\mathrm{Pct}$ = percent contribution to energy intake or specific nutrient intake, as appropriate. 


\subsection{Contribution of Foods to Percent Calcium Intake}

Total mean daily calcium intake was $971.5 \pm 23.8 \mathrm{mg} ; 1074.5 \pm 19.1 \mathrm{mg}$; and $1056.9 \pm 21.2$ for children 2-5, 6-11, and 12-18 years, respectively. Table 3 shows the food sources contributing at least $1 \%$ of total calcium intake. There were 20,19, and 21 food sources that contributed at least $1 \%$ of SFG calcium intake of children 2-5, 6-11, and 12-18 years, respectively. Using SFG intake data for children 2-5 years, milk (318 $\mathrm{mg} ; 32.7 \%$ of calcium), cheese (99 mg; 10.2\%), and flavored milk (74 mg; $7.6 \%$ ) were ranked as the top food sources of calcium. Using adjusted data, the rank order in children 2-5 years remained the same; however the percentages changed for: milk (352 mg; 36.2\%; +34 mg) and cheese (190 mg; 19.5\%; +90.6 mg), but not for flavored milk (74 mg; 7.6\%; $0.0 \mathrm{mg}$ ).

The top three SFG intake sources of calcium for children 6-11 years were milk ( $238 \mathrm{mg} ; 22.2 \%$ ), cheese $(107 \mathrm{mg} ; 10.0 \%)$, and pizza ( $89 \mathrm{mg} ; 5.7 \%)$. Using adjusted data, milk remained the top source of calcium $(287 ; 26.7 \% ;+49.1 \mathrm{mg})$, with cheese ranked second $(282 \mathrm{mg} ; 26.2 \% ;+174.6 \mathrm{mg})$; flavored milk ranked third ( $88 \mathrm{mg} ; 8.2 \% ; 0.0 \mathrm{mg})$. Pizza dropped to the 20th source of calcium $(10 \mathrm{mg} ; 0.9 \%$; +175 mg).

Milk (240 mg; $22.7 \%$ ), cheese (114 mg; $10.8 \%$ ), and pizza ( $86 \mathrm{mg} ; 8.1 \%$ ) were the SFG intake top food sources of calcium in children 12-18 years. Using adjusted data, cheese was the top ranked food (301 mg; 28.5\%; +187 mg), followed by milk ( $290 \mathrm{mg} ; 27.4 \%$; $+50 \mathrm{mg}$ ); pizza, the third rank food in the SFG data, fell to $23 \mathrm{rd}(7 \mathrm{mg} ; 0.7 \% ;-79 \mathrm{mg})$ and bread, rolls, and tortillas was the third most common source of calcium (56 mg; $5.3 \% ;-1 \mathrm{mg}$ ). 
Table 3. Food/food group sources ${ }^{1}$ of mean calcium $(\mathrm{mg})$ intake among US children aged $2-18$ years $(\mathrm{N}=5876)$ : National Health and Nutrition Examination Survey 2011-2014.

\begin{tabular}{|c|c|c|c|c|c|c|c|c|c|c|c|c|c|c|c|c|c|}
\hline \multicolumn{18}{|c|}{ Mean Calcium Intake (mg) of Children 2-5 Years of Age $(n=1511)$} \\
\hline \multirow[b]{2}{*}{ Sub Group Description } & \multicolumn{6}{|c|}{ Specific Food Group Intake } & \multicolumn{6}{|c|}{ Adjusted Intake ${ }^{2}$} & \multicolumn{5}{|c|}{ Delta Intake } \\
\hline & Cons & Rank & Mean & SE & Pct & SE & Cons & Rank & Mean & SE & Pct & SE & Cons & Mean & SE & Pct & SE \\
\hline Milk & 1120 & 1 & 317.6 & 12.0 & 32.7 & 0.8 & 1415 & 1 & 351.6 & 11.8 & 36.2 & 0.8 & 1120 & 34.0 & 1.7 & 3.5 & 0.2 \\
\hline Cheese & 542 & 2 & 98.9 & 11.6 & 10.2 & 1.0 & 1047 & 2 & 189.5 & 13.2 & 19.5 & 1.1 & 820 & 90.6 & 4.5 & 9.3 & 0.4 \\
\hline Flavored Milk & 253 & 3 & 74.0 & 8.9 & 7.6 & 0.9 & 253 & 3 & 74.0 & 8.9 & 7.6 & 0.9 & 0 & 0.0 & 0.0 & 0.0 & 0.0 \\
\hline Breads, Rolls, Tortillas & 818 & 4 & 43.2 & 3.2 & 4.5 & 0.3 & 818 & 5 & 42.8 & 3.1 & 4.4 & 0.3 & 30 & -0.4 & 0.3 & -0.0 & 0.0 \\
\hline Yogurt & 231 & 5 & 41.7 & 4.7 & 4.3 & 0.5 & 278 & 4 & 43.2 & 4.7 & 4.5 & 0.5 & 60 & 1.5 & 0.6 & 0.2 & 0.1 \\
\hline $100 \%$ Juice & 755 & 6 & 40.4 & 3.6 & 4.2 & 0.4 & 755 & 6 & 40.4 & 3.6 & 4.2 & 0.4 & 0 & 0.0 & 0.0 & 0.0 & 0.0 \\
\hline Mixed Dishes-Mexican & 201 & 7 & 39.7 & 4.8 & 4.1 & 0.5 & 201 & 16 & 9.2 & 1.1 & 0.9 & 0.1 & 189 & -30.6 & 3.9 & -3.1 & 0.4 \\
\hline Mixed Dishes-Pizza & 247 & 8 & 35.9 & 4.1 & 3.7 & 0.4 & 247 & 33 & 2.3 & 0.4 & 0.2 & 0.0 & 247 & -33.6 & 4.0 & -3.5 & 0.4 \\
\hline Mixed Dishes-Grain-based & 466 & 9 & 27.8 & 2.2 & 2.9 & 0.2 & 466 & 14 & 10.3 & 1.3 & 1.1 & 0.1 & 266 & -17.5 & 1.7 & -1.8 & 0.2 \\
\hline Ready-to-Eat Cereals & 695 & 10 & 25.5 & 1.9 & 2.6 & 0.2 & 695 & 7 & 25.4 & 1.9 & 2.6 & 0.2 & 12 & -0.0 & 0.0 & 0.0 & 0.0 \\
\hline Plain Water & 1229 & 11 & 20.8 & 1.3 & 2.1 & 0.1 & 1229 & 8 & 20.8 & 1.3 & 2.1 & 0.1 & 0 & 0.0 & 0.0 & 0.0 & 0.0 \\
\hline Other Desserts & 416 & 12 & 18.1 & 2.3 & 1.9 & 0.2 & 416 & 18 & 8.0 & 1.1 & 0.8 & 0.1 & 291 & -10.1 & 1.3 & -1.0 & 0.1 \\
\hline Quick Breads and Bread Products & 293 & 13 & 18.0 & 2.4 & 1.9 & 0.3 & 293 & 10 & 13.7 & 1.9 & 1.4 & 0.2 & 274 & -4.3 & 0.8 & -0.4 & 0.1 \\
\hline Dairy Drinks and Substitutes & 70 & 14 & 16.5 & 3.4 & 1.7 & 0.4 & 70 & 9 & 16.5 & 3.4 & 1.7 & 0.4 & 0 & 0.0 & 0.0 & 0.0 & 0.0 \\
\hline Mixed Dishes-Sandwiches & 208 & 15 & 15.2 & 1.8 & 1.6 & 0.2 & 208 & 12 & 10.5 & 1.3 & 1.2 & 0.1 & 67 & -4.7 & 0.9 & -0.5 & 0.1 \\
\hline Eggs & 332 & 16 & 13.0 & 1.5 & 1.3 & 0.2 & 332 & 20 & 6.3 & 0.6 & 0.7 & 0.1 & 234 & -6.7 & 1.2 & -0.7 & 0.1 \\
\hline Sweet Bakery Products & 723 & 17 & 12.7 & 1.2 & 1.3 & 0.1 & 723 & 13 & 10.4 & 0.9 & 1.1 & 0.1 & 262 & -2.3 & 0.3 & -0.2 & 0.0 \\
\hline Fruit & 945 & 18 & 10.8 & 0.5 & 1.1 & 0.1 & 945 & 11 & 10.8 & 0.5 & 1.1 & 0.1 & 2 & 0.0 & 0.0 & 0.0 & 0.0 \\
\hline Vegetables, excluding Potatoes & 560 & 19 & 10.6 & 1.0 & 1.1 & 0.1 & 560 & 15 & 9.8 & 1.1 & 1.0 & 0.1 & 22 & -0.8 & 0.5 & -0.1 & 0.1 \\
\hline Cooked Cereals & 125 & 20 & 10.4 & 1.5 & 1.1 & 0.2 & 125 & 22 & 6.1 & 0.9 & 0.6 & 0.1 & 69 & -4.3 & 1.0 & -0.4 & 0.1 \\
\hline
\end{tabular}


Table 3. Cont

\begin{tabular}{|c|c|c|c|c|c|c|c|c|c|c|c|c|c|c|c|c|c|}
\hline \multicolumn{18}{|c|}{ Mean Calcium Intake (mg) of Children 6-11 Years of Age $(n=2193)$} \\
\hline \multirow[b]{2}{*}{ Sub Group Description } & \multicolumn{6}{|c|}{ Specific Food Group Intake } & \multicolumn{6}{|c|}{ Adjusted Intake } & \multicolumn{5}{|c|}{ Delta Intake } \\
\hline & Cons & Rank & Mean & SE & Pct & SE & Cons & Rank & Mean & SE & Pct & SE & Cons & Mean & SE & Pct & SE \\
\hline Milk & 1274 & 1 & 238.2 & 8.7 & 22.2 & 0.7 & 1987 & 1 & 287.3 & 8.9 & 26.7 & 0.8 & 1646 & 49.1 & 2.2 & 4.6 & 0.2 \\
\hline Cheese & 723 & 2 & 107.1 & 9.5 & 10.0 & 0.9 & 1643 & 2 & 281.7 & 12.3 & 26.2 & 0.9 & 1349 & 174.6 & 12.3 & 16.3 & 1.0 \\
\hline Mixed Dishes_Pizza & 548 & 3 & 88.9 & 9.8 & 8.3 & 0.9 & 548 & 20 & 9.5 & 1.8 & 0.9 & 0.2 & 547 & -79.3 & 9.3 & -7.4 & 0.8 \\
\hline Flavored Milk & 555 & 4 & 88.3 & 7.7 & 8.2 & 0.7 & 555 & 3 & 88.3 & 7.7 & 8.2 & 0.7 & 0 & 0.0 & 0.0 & 0.0 & 0.0 \\
\hline Mixed Dishes-Mexican & 362 & 5 & 67.9 & 8.1 & 6.3 & 0.8 & 362 & 11 & 16.2 & 2.0 & 1.5 & 0.2 & 347 & -51.7 & 6.5 & -4.8 & 0.6 \\
\hline Breads, Rolls, Tortillas & 1205 & 6 & 59.6 & 3.0 & 5.6 & 0.3 & 1205 & 4 & 58.6 & 2.9 & 5.5 & 0.3 & 85 & -1.1 & 0.2 & -0.1 & 0.0 \\
\hline Mixed Dishes-Grain-based & 606 & 7 & 39.0 & 4.5 & 3.6 & 0.4 & 606 & 14 & 13.6 & 1.4 & 1.3 & 0.1 & 324 & -25.4 & 3.7 & -2.4 & 0.3 \\
\hline Other Desserts & 620 & 8 & 35.1 & 3.1 & 3.3 & 0.3 & 620 & 13 & 14.7 & 1.3 & 1.4 & 0.1 & 478 & -20.4 & 2.0 & -1.9 & 0.2 \\
\hline $100 \%$ Juice & 759 & 9 & 34.8 & 5.1 & 3.2 & 0.5 & 759 & 5 & 34.8 & 5.1 & 3.2 & 0.5 & 0 & 0.0 & 0.0 & 0.0 & 0.0 \\
\hline Mixed Dishes-Sandwiches & 436 & 10 & 31.2 & 3.6 & 2.9 & 0.3 & 436 & 10 & 19.3 & 2.8 & 1.8 & 0.3 & 170 & -12.2 & 1.7 & -1.1 & 0.2 \\
\hline Quick Breads and Bread Products & 475 & 11 & 29.6 & 2.7 & 2.8 & 0.3 & 475 & 9 & 23.5 & 2.5 & 2.2 & 0.2 & 445 & -6.1 & 0.5 & -0.6 & 0.0 \\
\hline Ready-to-Eat Cereals & 861 & 12 & 28.2 & 2.4 & 2.6 & 0.2 & 861 & 6 & 28.1 & 2.4 & 2.6 & 0.2 & 24 & -0.1 & 0.1 & -0.0 & 0.0 \\
\hline Plain Water & 1732 & 13 & 27.9 & 1.4 & 2.6 & 0.1 & 1732 & 7 & 27.9 & 1.4 & 2.6 & 0.1 & 0 & 0.0 & 0.0 & 0.0 & 0.0 \\
\hline Yogurt & 206 & 14 & 22.7 & 2.6 & 2.1 & 0.2 & 281 & 8 & 26.9 & 2.8 & 2.5 & 0.3 & 93 & 4.3 & 1.1 & 0.4 & 0.1 \\
\hline Sweet Bakery Products & 1034 & 15 & 18.9 & 2.0 & 1.8 & 0.2 & 1034 & 12 & 15.3 & 1.5 & 1.4 & 0.2 & 455 & -3.6 & 0.5 & -0.3 & 0.1 \\
\hline Sweetened Beverages & 1467 & 16 & 15.3 & 2.1 & 1.4 & 0.2 & 1467 & 16 & 12.7 & 1.2 & 1.2 & 0.1 & 45 & -2.6 & 1.2 & -0.2 & 0.1 \\
\hline Dairy Drinks and Substitutes & 81 & 17 & 13.4 & 2.5 & 1.2 & 0.2 & 81 & 15 & 13.4 & 2.5 & 1.2 & 0.2 & 0 & 0.0 & 0.0 & 0.0 & 0.0 \\
\hline Fruits & 1153 & 18 & 11.5 & 0.6 & 1.1 & 0.1 & 1153 & 17 & 11.5 & 0.5 & 1.1 & 0.1 & 6 & -0.0 & 0.0 & 0.0 & 0.0 \\
\hline Vegetables, excluding Potatoes & 792 & 19 & 10.9 & 1.0 & 1.0 & 0.1 & 792 & 18 & 9.9 & 1.0 & 0.9 & 0.1 & 40 & -1.0 & 0.3 & -0.1 & 0.0 \\
\hline
\end{tabular}


Table 3. Cont

\begin{tabular}{|c|c|c|c|c|c|c|c|c|c|c|c|c|c|c|c|c|c|}
\hline \multicolumn{18}{|c|}{ Mean Calcium Intake (mg) of Children 12-18 Years of Age $(n=2172)$} \\
\hline \multirow[b]{2}{*}{ Sub Group Description } & \multicolumn{6}{|c|}{ Specific Food Group Intake } & \multicolumn{6}{|c|}{ Adjusted Intake } & \multicolumn{5}{|c|}{ Delta Intake } \\
\hline & Cons & Rank & Mean & SE & Pct & SE & Cons & Rank & Mean & SE & Pct & SE & Cons & Mean & SE & Pct & SE \\
\hline Milk & 973 & 1 & 239.8 & 12.5 & 22.7 & 1.0 & 1779 & 2 & 289.9 & 12.6 & 27.4 & 0.9 & 1453 & 50.1 & 2.6 & 4.0 & 0.2 \\
\hline Cheese & 687 & 2 & 113.6 & 5.4 & 10.8 & 0.6 & 1565 & 1 & 300.7 & 12.1 & 28.5 & 0.9 & 1276 & 187.2 & 12.1 & 17.7 & 0.9 \\
\hline Mixed Dishes_-Pizza & 486 & 3 & 85.6 & 8.9 & 8.1 & 0.8 & 486 & 23 & 6.9 & 1.0 & 0.7 & 0.1 & 486 & -78.7 & 8.2 & -7.5 & 0.7 \\
\hline Mixed Dishes-Mexican & 329 & 4 & 68.1 & 8.7 & $\begin{array}{l}0.1 \\
6.4\end{array}$ & 0.8 & 329 & 10 & 16.9 & 2.0 & 1.6 & 0.2 & 313 & -51.2 & 7.1 & -4.8 & 0.6 \\
\hline Breads, Rolls, Tortillas & 1119 & 5 & 57.3 & 3.2 & 5.4 & 0.3 & 1119 & 3 & 56.1 & 3.3 & 5.3 & 0.3 & 85 & -1.2 & 0.2 & -0.1 & 0.0 \\
\hline Plain Water & 1673 & 6 & 53.1 & 3.1 & 5.0 & 0.3 & 1673 & 4 & 53.1 & 3.1 & 5.0 & 0.3 & 0 & 0.0 & 0.0 & 0. & 0.0 \\
\hline Flavored Milk & 225 & 7 & 40.5 & 5.1 & 3.8 & 0.5 & 225 & 5 & 40.5 & 5.1 & 3.8 & 0.5 & 0 & 0.0 & 0.0 & 0.0 & 0.0 \\
\hline Mixed Dishes-Sandwiches & 374 & 8 & 40.1 & 4.9 & 3.8 & 0.5 & 374 & 8 & 21.5 & 2.4 & 2.0 & 0.2 & 174 & -18.7 & 3.4 & -1.8 & 0.3 \\
\hline Mixed Dishes-Grain-based & 464 & 9 & 37.1 & 3.9 & 3.5 & 0.4 & 464 & 15 & 12.1 & 1.3 & 1.2 & 0.1 & 244 & -25.0 & 3.4 & -2.4 & 0.3 \\
\hline Other Desserts & 377 & 10 & 30.9 & 4.4 & 2.9 & 0.4 & 377 & 16 & 12.0 & 1.3 & 1.1 & 0.1 & 339 & -18.9 & 3.3 & -1.8 & 0.3 \\
\hline $100 \%$ Juice & 487 & 11 & 28.1 & 4.0 & 2.7 & 0.4 & 487 & 6 & 28.1 & 4.0 & 2.7 & 0.4 & 0 & 0.0 & 0.0 & 0.0 & 0.0 \\
\hline Ready-to-Eat Cereals & 582 & 12 & 27.3 & 4.4 & 2.6 & 0.4 & 582 & 7 & 27.2 & 4.4 & 2.6 & 0.4 & 18 & -0.1 & 0.0 & -0.0 & 0.0 \\
\hline Quick Breads and Bread Products & 297 & 13 & 21.3 & 2.6 & 2.0 & 0.2 & 297 & 9 & 17.0 & 2.3 & 1.6 & 0.2 & 282 & -4.2 & 0.4 & -0.4 & 0.0 \\
\hline Sweet Bakery Products & 833 & 14 & 16.6 & 1.4 & 1.6 & 0.1 & 833 & 13 & 13.3 & 1.1 & 1.3 & 0.1 & 344 & -3.3 & 0.4 & -0.3 & 0.0 \\
\hline Sweetened Beverages & 1404 & 15 & 15.3 & 1.4 & 1.5 & 0.1 & 1404 & 14 & 12.9 & 1.1 & 1.2 & 0.1 & 40 & -2.3 & 0.6 & -0.2 & 0.1 \\
\hline White Potatoes & 580 & 16 & 14.6 & 2.1 & 1.4 & 0.2 & 580 & 28 & 4.9 & 0.4 & 0.5 & 0.0 & 121 & -9.7 & 1.8 & -0.9 & 0.2 \\
\hline Dairy Drinks and Substitutes & 79 & 17 & 14.5 & 1.8 & $\begin{array}{l}1.7 \\
1.4\end{array}$ & 0.2 & 79 & 11 & 14.5 & $\begin{array}{l}0.7 \\
1.8\end{array}$ & 1.4 & 0.2 & 0 & 0.0 & 0.0 & 0.0 & 0.0 \\
\hline Savory Snacks & 942 & 18 & 12.5 & 0.9 & 1.2 & 0.1 & 942 & 17 & 11.2 & 0.8 & 1.1 & 0.1 & 233 & -1.3 & 0.2 & -0.1 & 0.0 \\
\hline Eggs & 333 & 19 & 12.2 & 1.1 & 1.2 & 0.1 & 333 & 24 & 6.6 & 0.5 & 0.6 & 0.1 & 211 & -5.6 & 0.7 & -0.5 & 0.1 \\
\hline Vegetables, excluding Potatoes & 803 & 20 & 12.2 & 1.2 & 1.2 & 0.1 & 803 & 18 & 11.2 & 1.2 & 1.1 & 0.1 & 28 & -1.0 & 0.3 & -0.1 & 0.0 \\
\hline Mixed Dishes-Meat, Poultry, Fish & 288 & 21 & 12.1 & 1.5 & 1.2 & 0.1 & 288 & 25 & 6.5 & 0.7 & 0.6 & 0.1 & 100 & -5.7 & 1.1 & -0.5 & 0.1 \\
\hline
\end{tabular}

${ }^{1}$ To a $1 \%$ contribution of daily intake of calcium; ${ }^{2}$ Nutrients from milk, cheese, and yogurt for non-dairy foods are added to the nutrients in the milk, cheese, and yogurt food categories,

respectively. For non-dairy foods the nutrients displayed are only for the milk, cheese, and yogurt in the non-dairy food. Abbreviations: Cons = consumers, $\mathrm{M} / \mathrm{P} / \mathrm{F}=\mathrm{meat} / \mathrm{poultry} / \mathrm{fish}$;

$\mathrm{SE}=$ standard error; Pct = percent contribution to energy intake or specific nutrient intake, as appropriate. 


\subsection{Contribution of Foods to Percent Vitamin D Intake}

Total mean daily vitamin D intake was $6.2 \pm 0.2 \mathrm{mcg} ; 5.7 \pm 0.1 \mathrm{mcg}$; and $5.3 \pm 0.2 \mathrm{mcg}$, respectively, for children 2-5, 6-11, and 12-18 years. Table 4 shows the food sources contributing at least $1 \%$ of vitamin D intake. There were 11, 14, and 14 different SFG sources that contributed at least $1 \%$ of the vitamin D intake of children 2-5, 6-11, and 12-18 years, respectively. Using SFG intake data for children 2-5 years, milk (3.3 mcg; $52.6 \% ; 0.2 \mathrm{mcg}$ ), flavored milk ( $0.8 \mathrm{mcg} ; 12.0 \% ; 0.0 \mathrm{mcg}$ ), and $(0.5 \mathrm{mcg} ; 7.5 \%)$ were the top food sources of vitamin D. Using adjusted data, the rank order remained the same with milk ( $3.5 \mathrm{mcg} ; 55.7 \%$; $+0.2 \mathrm{mcg}$ ), flavored milk (12 mcg; $12 \%)$, and RTEC $(7.5 \mathrm{mcg} ; 7.5 \%$; $0.0 \mathrm{mcg}$ ). Eggs were the highest-ranking (fourth) non-fortified SFG food group consumed by this age group ( $0.3 \mathrm{mcg} ; 4.4 \%)$; however, after adjustment, eggs fell to fifth with a mean of $0.2 \mathrm{mcg} ; 3.8 \%$.

For children 6-11 years, milk ( $2.7 \mathrm{mcg}$; $46.7 \%)$, flavored milk ( $0.9 \mathrm{mcg} ; 15.2 \%)$, and RTEC ( $0.6 \mathrm{mcg}$; $9.9 \%$ ) were the top SFG sources of vitamin D, respectively. Using adjusted data, the rank order remained $(2.7 \mathrm{mcg} ; 46.7 \%$; $+0.3 \mathrm{mcg}$ ), flavored milk ( $0.9 \mathrm{mcg} ; 15.2 \%$; $0.0 \mathrm{mcg})$, and RTEC (0.6 mcg; $9.9 \% ; 0.0 \mathrm{mcg})$. Eggs were the highest-ranking non-fortified SFG and adjusted food source of vitamin $\mathrm{D}(0.2 \mathrm{mcg} ; 4.1 \%$ and $0.2 \mathrm{mcg} ; 3.5 \%)$, respectively.

For children $12-18$ years, milk ( $2.4 \mathrm{mcg} ; 45.6 \%)$, RTEC ( $0.5 \mathrm{mcg} ; 9.2 \%)$, and flavored milk $(0.4 \mathrm{mcg}$; $7.6 \%)$, were the top three SFG food sources of vitamin D. Using adjusted data, milk ( $2.7 \mathrm{mcg} ; 51.3 \%$; $+0.3 \mathrm{mcg}$ ), cheese $(0.5 \mathrm{mcg} ; 9.6 \% ;+0.2 \mathrm{mcg})$, and RTEC ( $0.9 \mathrm{mcg} ; 9.1 \% ; 0.0 \mathrm{mcg})$ were the top three food sources of vitamin D.

\subsection{Contribution of Foods to Percent Potassium Intake}

Total mean daily potassium intake was $1981.8 \pm 39.5 \mathrm{mg} ; 2197.9 \pm 27.0 \mathrm{mg} ;$ and $2308.2 \pm 44.9 \mathrm{mg}$ for children 2-5, 6-11, and 12-18 years, respectively. Table 5 shows the food sources contributing at least $1 \%$ of potassium intake. There were 25,25 , and 26 food sources that contributed at least $1 \%$ of potassium intake of children 2-5, 6-11, and 12-18 years, respectively. Using SFG intake data for children $2-5$ years, milk ( $375 \mathrm{mg} ; 18.9 \%$ ), fruit (190 mg; 9.6\%), and 100\% fruit juice (169 mg; 8.5\%) were the top food sources of potassium. When the data were adjusted, the rank order of the top food sources of potassium remained the same: milk ( $417 \mathrm{mg} ; 21.1 \%$; $+41.8 \mathrm{mg})$, fruit (190 mg; $9.6 \%$; delta $0 \mathrm{mg}$ ), and $100 \%$ fruit juice ( $169 \mathrm{mg} ; 8.5 \%$; delta $0 \mathrm{mg}$ ).

In children 6-11 years, milk ( $283 \mathrm{mg} ; 12.9 \%)$, fruit (160 mg; 7.3\%), and flavored milk (125 mg; $5.7 \%$ ) were the three SFG top sources of potassium. Using adjusted data, the rank order remained the same with milk (344 mg; $15.6 \%$; $+60 \mathrm{mg})$, fruit (160 mg; $7.3 \% ;-0.1)$, and flavored milk (125 mg; 5.7\%; $0 \mathrm{~g}$ ) as the top three sources of potassium intake.

In the oldest group of children, the top SFG food sources of potassium were milk (milk $286 \mathrm{mg}$; $12.4 \%$ ), white potatoes (145 mg; $6.3 \%)$, and fruit ( $129 \mathrm{mg} ; 5.6 \%)$. The rank order remained the same for adjusted data, with milk ( $347 \mathrm{mg} ; 15 \%$; $+61 \mathrm{mg}$ ), white potatoes (141 mg; $6.1 \%$; $-4 \mathrm{mg}$ ), and fruit (129 mg; 5.6\%; $0.0 \mathrm{mcg}$ ), respectively. 
Table 4. Food/food group sources ${ }^{1}$ of mean vitamin D intake (mcg) among US children aged 2-18 years ( $\left.N=5876\right)$ : National Health and Nutrition Examination Survey 2011-2014.

\begin{tabular}{|c|c|c|c|c|c|c|c|c|c|c|c|c|c|c|c|c|c|}
\hline \multicolumn{18}{|c|}{ Mean Vitamin D Intake $(\mathrm{mcg})$ of Children 2-5 Years of Age $(n=1511)$} \\
\hline WWEIA Food Group & \multicolumn{6}{|c|}{ Specific Food Group Intake } & \multicolumn{6}{|c|}{ Adjusted Intake ${ }^{2}$} & \multicolumn{5}{|c|}{ Delta Intake } \\
\hline Sub Group Description & Cons & Rank & Mean & SE & Pct & SE & Cons & Rank & Mean & SE & Pct & SE & Cons & Mean & SE & Pct & SE \\
\hline Milk & 1120 & 1 & 3.3 & 0.1 & 52.6 & 1.5 & 1415 & 1 & 3.5 & 0.1 & 55.7 & 1.5 & 1120 & 0.2 & 0.0 & 3.0 & 0.2 \\
\hline Flavored Milk & 253 & 2 & 0.8 & 0.1 & 12.0 & 1.4 & 253 & 2 & 0.8 & 0.1 & 12.0 & 1.4 & 0 & 0.0 & 0.0 & 0.0 & 0.0 \\
\hline Ready-to-Eat Cereals & 695 & 3 & 0.5 & 0.0 & 7.5 & 0.5 & 695 & 3 & 0.5 & 0.0 & 7.5 & 0.5 & 12 & 0.0 & 0.0 & 0.0 & 0.0 \\
\hline Eggs & 332 & 4 & 0.3 & 0.0 & 4.4 & 0.5 & 332 & 5 & 0.2 & 0.0 & 3.8 & 0.4 & 234 & 0.0 & 0.0 & -0.7 & 0.1 \\
\hline Cheese & 542 & 5 & 0.3 & 0.0 & 4.2 & 0.4 & 1047 & 4 & 0.3 & 0.0 & 5.4 & 0.5 & 820 & 0.1 & 0.0 & 1.2 & 0.1 \\
\hline Seafood & 96 & 6 & 0.2 & 0.1 & 3.2 & 1.3 & 96 & 6 & 0.2 & 0.1 & 3.2 & 1.3 & 26 & 0.0 & 0.0 & 0.0 & 0.0 \\
\hline Yogurt & 231 & 7 & 0.2 & 0.0 & 2.4 & 0.3 & 278 & 7 & 0.2 & 0.0 & 2.4 & 0.3 & 60 & 0.0 & 0.0 & 0.0 & 0.0 \\
\hline $100 \%$ Juice & 755 & 8 & 0.1 & 0.0 & 1.8 & 0.3 & 755 & 8 & 0.1 & 0.0 & 1.8 & 0.3 & 0 & 0.0 & 0.0 & 0.0 & 0.0 \\
\hline Cured Meats/Poultry & 459 & 9 & 0.1 & 0.0 & 1.7 & 0.2 & 459 & 9 & 0.1 & 0.0 & 1.7 & 0.2 & 2 & 0.0 & 0.0 & 0.0 & 0.0 \\
\hline Dairy Drinks and Substitutes & 70 & 10 & 0.1 & 0.0 & 1.7 & 0.4 & 70 & 10 & 0.1 & 0.0 & 1.7 & 0.4 & 0 & 0.0 & 0.0 & 0.0 & 0.0 \\
\hline Mixed Dishes-Grain-based & 466 & 11 & 0.1 & 0.0 & 1.3 & 0.1 & 466 & 15 & 0.0 & 0.0 & 0.4 & 0.1 & 266 & -0.1 & 0.0 & -1.0 & 0.1 \\
\hline \multicolumn{18}{|c|}{ Mean Vitamin D Intake (mcg) of Children 6-11 Years of Age $(n=2193)$} \\
\hline WWEIA Food Group & \multicolumn{6}{|c|}{ Specific Food Group Intake } & \multicolumn{6}{|c|}{ Adjusted Intake } & \multicolumn{5}{|c|}{ Delta Intake } \\
\hline Sub Group Description & Cons & Rank & Mean & SE & Pct & SE & Cons & Rank & Mean & $\mathrm{SE}$ & Pct & SE & Cons & Mean & SE & Pct & SE \\
\hline Milk & 1274 & 1 & 2.4 & 0.1 & 42.3 & 1.1 & 1987 & 1 & 2.7 & 0.1 & 46.7 & 1.1 & 1646 & 0.3 & 0.0 & 4.5 & 0.4 \\
\hline Flavored Milk & 555 & 2 & 0.9 & 0.1 & 15.2 & 1.2 & 555 & 2 & 0.9 & 0.1 & 15.2 & 1.2 & 0 & 0.0 & 0.0 & 0.0 & 0.0 \\
\hline Ready-to-Eat Cereals & 861 & 3 & 0.6 & 0.0 & 9.9 & 0.6 & 861 & 3 & 0.6 & 0.0 & 9.9 & 0.6 & 24 & 0.0 & 0.0 & 0.0 & 0.0 \\
\hline Cheese & 723 & 4 & 0.4 & 0.0 & 6.0 & 0.7 & 1643 & 4 & 0.5 & 0.0 & 8.4 & 0.7 & 1349 & 0.1 & 0.0 & 2.4 & 0.2 \\
\hline Eggs & 324 & 5 & 0.2 & 0.0 & 4.1 & 0.4 & 324 & 5 & 0.2 & 0.0 & 3.5 & 0.4 & 217 & 0.0 & 0.0 & -0.6 & 0.1 \\
\hline Seafood & 151 & 6 & 0.2 & 0.0 & 2.6 & 0.5 & 151 & 6 & 0.2 & 0.0 & 2.6 & 0.5 & 47 & 0.0 & 0.0 & 0.0 & 0.0 \\
\hline $100 \%$ Juice & 759 & 7 & 0.1 & 0.0 & 2.1 & 0.4 & 759 & 7 & 0.1 & 0.0 & 2.1 & 0.4 & 0 & 0.0 & 0.0 & 0.0 & 0.0 \\
\hline Cured Meats/Poultry & 687 & 8 & 0.1 & 0.0 & 1.9 & 0.2 & 687 & 8 & 0.1 & 0.0 & 1.9 & 0.2 & 1 & 0.0 & 0.0 & 0.0 & 0.0 \\
\hline Mixed Dishes-Sandwiches & 436 & 9 & 0.1 & 0.0 & 1.8 & 0.2 & 436 & 10 & 0.1 & 0.0 & 1.4 & 0.2 & 170 & 0.0 & 0.0 & 0.4 & 0.1 \\
\hline Mixed Dishes-Grain-based & 606 & 10 & 0.1 & 0.0 & 1.7 & 0.2 & 606 & 16 & 0.0 & 0.0 & 0.4 & 0.1 & 324 & -0.1 & 0.0 & 1.3 & 0.2 \\
\hline Yogurt & 206 & 11 & 0.1 & 0.0 & 1.5 & 0.2 & 281 & 9 & 0.1 & 0.0 & 1.6 & 0.2 & 93 & 0.0 & 0.0 & 0.1 & 0.1 \\
\hline Dairy Drinks and Substitutes & 81 & 12 & 0.1 & 0.0 & 1.3 & 0.3 & 81 & 11 & 0.1 & 0.0 & 1.3 & 0.3 & 0 & 0.0 & 0.0 & 0.0 & 0.0 \\
\hline Mixed Dishes-M/P/F & 281 & 13 & 0.1 & 0.0 & 1.2 & 0.4 & 281 & 13 & 0.1 & 0.0 & 0.8 & 0.3 & 123 & 0.0 & 0.0 & -0.4 & 0.1 \\
\hline Quick Breads and Bread Products & 475 & 14 & 0.1 & 0.0 & 1.0 & 0.2 & 475 & 15 & 0.0 & 0.0 & 0.5 & 0.1 & 445 & 0.0 & 0.0 & -0.6 & 0.1 \\
\hline
\end{tabular}


Table 4. Cont.

\begin{tabular}{|c|c|c|c|c|c|c|c|c|c|c|c|c|c|c|c|c|c|}
\hline \multicolumn{18}{|c|}{ Mean Vitamin D Intake $(\mathrm{mcg})$ of Children $12-18$ Years of Age $(n=2172)$} \\
\hline \multirow{2}{*}{$\begin{array}{c}\text { WWEIA Food Group } \\
\text { Sub Group Description }\end{array}$} & \multicolumn{6}{|c|}{ Actual Intake } & \multicolumn{6}{|c|}{ Adjusted Intake } & \multicolumn{5}{|c|}{ Delta Intake } \\
\hline & Cons & Rank & Mean & SE & Pct & SE & Cons & Rank & Mean & SE & Pct & SE & Cons & Mean & SE & Pct & SE \\
\hline Milk & 973 & 1 & 2.4 & 0.1 & 45.6 & 1.6 & 1779 & 1 & 2.7 & 0.1 & 51.3 & 1.5 & 1453 & 0.3 & 0.0 & 5.7 & 0.4 \\
\hline Ready-to-Eat Cereals & 582 & 2 & 0.5 & 0.0 & 9.2 & 0.7 & 582 & 3 & 0.5 & 0.0 & 9.1 & 0.7 & 18 & 0.0 & 0.0 & 0.0 & 0.0 \\
\hline Flavored Milk & 225 & 3 & 0.4 & 0.1 & 7.6 & 1.0 & 225 & 4 & 0.4 & 0.1 & 7.6 & 1.0 & 0 & 0.0 & 0.0 & 0.0 & 0.0 \\
\hline Cheese & 687 & 4 & 0.3 & 0.0 & 6.4 & 0.4 & 1565 & 2 & 0.5 & 0.0 & 9.6 & 0.5 & 1276 & 0.2 & 0.0 & 3.1 & 0.2 \\
\hline Eggs & 333 & 5 & 0.3 & 0.0 & 5.2 & 0.4 & 333 & 5 & 0.2 & 0.0 & 4.6 & 0.3 & 211 & 0.0 & 0.0 & -0.7 & 0.2 \\
\hline Seafood & 142 & 6 & 0.2 & 0.1 & 3.7 & 1.1 & 142 & 6 & 0.2 & 0.1 & 3.7 & 1.1 & 21 & 0.00 & 0.0 & 0.0 & 0.0 \\
\hline Cured Meats/Poultry & 585 & 7 & 0.1 & 0.0 & 2.3 & 0.3 & 585 & 7 & 0.1 & 0.0 & 2.3 & 0.3 & 2 & 0.00 & 0.0 & 0.0 & 0.0 \\
\hline Mixed Dishes-Grain-based & 464 & 8 & 0.1 & 0.0 & 2.0 & 0.3 & 464 & 16 & 0.0 & 0.0 & 0.6 & 0.1 & 244 & -0.1 & 0.0 & -1.5 & 0.2 \\
\hline Mixed Dishes-Sandwiches & 374 & 9 & 0.1 & 0.0 & 2.0 & 0.3 & 374 & 9 & 0.1 & 0.0 & 1.4 & 0.2 & 174 & 0.0 & 0.0 & -0.6 & 0.1 \\
\hline $100 \%$ Juice & 487 & 10 & 0.1 & 0.0 & 1.8 & 0.3 & 487 & 8 & 0.1 & 0.0 & 1.8 & 0.3 & 0 & 0.0 & 0.0 & 0.0 & 0.0 \\
\hline Mixed Dishes-M/P/F & 288 & 11 & 0.1 & 0.0 & 1.7 & 0.4 & 288 & 10 & 0.1 & 0.0 & 1.2 & 0.4 & 100 & 0.0 & 0.0 & -0.5 & 0.1 \\
\hline White Potatoes & 580 & 12 & 0.1 & 0.0 & 1.2 & 0.2 & 580 & 17 & 0.0 & 0.0 & 0.5 & 0.1 & 121 & 0.0 & 0.0 & -0.7 & 0.1 \\
\hline Coffee and Tea & 505 & 13 & 0.1 & 0.0 & 1.1 & 0.2 & 505 & 30 & 0.0 & 0.0 & 0.0 & 0.0 & 60 & -0.1 & 0.0 & -1.0 & 0.2 \\
\hline Poultry & 652 & 14 & 0.1 & 0.0 & 1.0 & 0.1 & 652 & 11 & 0.1 & 0.0 & 1.0 & 0.1 & 130 & 0.0 & 0.0 & -0.1 & 0.0 \\
\hline
\end{tabular}

Nutrients from milk, cheese, and yogurt for non-dairy foods are added to the nutrients in the milk, cheese, and yogurt food categories, respectively. For non-dairy foods the nutrients displayed are only for the milk, cheese, and yogurt in the non-dairy food. Abbreviations: Cons = consumers, $\mathrm{M} / \mathrm{P} / \mathrm{F}=\mathrm{meat} / \mathrm{poultry} /$ fish; $\mathrm{SE}=$ standard error; $\mathrm{Pct}=$ percent contribution to energy intake or specific nutrient intake, as appropriate. 
Table 5. Food/food group sources ${ }^{1}$ of mean potassium (mg) intake among US children aged 2-18 years $(N=5876)$ : National Health and Nutrition Examination Survey 2011-2014.

\begin{tabular}{|c|c|c|c|c|c|c|c|c|c|c|c|c|c|c|c|c|c|}
\hline \multicolumn{18}{|c|}{ Mean Potassium Intake (mg) of Children 2-5 Years of Age $(n=1511)$} \\
\hline \multirow{2}{*}{$\begin{array}{l}\text { WWEIA Food Group } \\
\text { Sub Group Description }\end{array}$} & \multicolumn{6}{|c|}{ Specific Food Group Intake } & \multicolumn{6}{|c|}{ Adjusted Intake ${ }^{2}$} & \multicolumn{5}{|c|}{ Delta Intake } \\
\hline & Cons & Rank & Mean & SE & Pct & SE & Cons & Rank & Mean & SE & Pct & SE & Cons & Mean & SE & Pct & SE \\
\hline Milk & 1120 & 1 & 375.4 & 14.4 & 18.9 & 0.6 & 1415 & 1 & 417.1 & 13.9 & 21.1 & 0.6 & 1120 & 41.8 & 2.1 & 2.1 & 0.1 \\
\hline Fruits & 945 & 2 & 189.7 & 7.6 & 9.6 & 0.4 & 945 & 2 & 189.7 & 7.6 & 9.6 & 0.4 & 2 & 0.00 & 0.0 & 0.0 & 0.0 \\
\hline 100\% Juice & 755 & 3 & 169.3 & 10.0 & 8.5 & 0.4 & 755 & 3 & 169.3 & 10.0 & 8.5 & 0.4 & 0 & 0.00 & 0.0 & 0.0 & 0.0 \\
\hline Flavored Milk & 253 & 4 & 100.6 & 13.1 & 5.1 & 0.6 & 253 & 4 & 100.6 & 13.1 & 5.1 & 0.6 & 0 & 0.00 & 0.0 & 0.0 & 0.0 \\
\hline Mixed Dishes-Grain-based & 466 & 5 & 80.1 & 8.0 & 4.0 & 0.4 & 466 & 7 & 72.9 & 7.9 & 3.7 & 0.4 & 266 & -7.2 & 0.8 & -0.4 & 0.0 \\
\hline Vegetables, excluding Potatoes & 560 & 6 & 78.5 & 7.2 & 4.0 & 0.4 & 560 & 5 & 78.0 & 7.3 & 3.9 & 0.4 & 22 & -0.5 & 0.2 & 0.0 & 0.0 \\
\hline White Potatoes & 401 & 7 & 78.3 & 7.6 & 4.0 & 0.4 & 401 & 6 & 76.7 & 7.5 & 3.9 & 0.4 & 78 & -1.6 & 0.5 & -0.1 & 0.0 \\
\hline Poultry & 526 & 8 & 69.9 & 6.6 & 3.5 & 0.3 & 526 & 8 & 69.7 & 6.6 & 3.5 & 0.3 & 71 & -0.1 & 0.0 & 0.0 & 0.0 \\
\hline Savory Snacks & 688 & 9 & 62.5 & 5.2 & 3.2 & 0.3 & 688 & 9 & 62.3 & 5.2 & 3.1 & 0.3 & 140 & -0.2 & 0.1 & 0.0 & 0.0 \\
\hline Sweetened Beverages & 787 & 10 & 60.9 & 5.5 & 3.1 & 0.3 & 787 & 10 & 59.8 & 5.4 & 3.0 & 0.3 & 23 & -1.1 & 0.4 & -0.1 & 0.0 \\
\hline Cured Meats/Poultry & 459 & 11 & 59.4 & 5.3 & 3.0 & 0.3 & 459 & 11 & 59.3 & 5.3 & 3.0 & 0.3 & 2 & -0.1 & 0.1 & 0.0 & 0.0 \\
\hline Yogurt & 231 & 12 & 53.6 & 6.0 & 2.7 & 0.3 & 278 & 12 & 55.6 & 5.9 & 2.8 & 0.3 & 60 & 2.0 & 0.7 & 0.1 & 0.0 \\
\hline Breads, Rolls, Tortillas & 818 & 13 & 48.9 & 3.6 & 2.5 & 0.2 & 818 & 13 & 48.8 & 3.6 & 2.5 & 0.2 & 30 & -0.1 & 0.1 & 0.0 & 0.0 \\
\hline Plant-Based Protein Foods & 346 & 14 & 46.8 & 4.6 & 2.4 & 0.2 & 346 & 14 & 46.8 & 4.6 & 2.4 & 0.2 & 1 & 0.0 & 0.0 & 0.0 & 0.0 \\
\hline Mixed Dishes-Mexican & 201 & 15 & 40.3 & 4.6 & 2.0 & 0.2 & 201 & 17 & 35.4 & 4.1 & 1.8 & 0.2 & 189 & -4.9 & 0.6 & -0.3 & 0.0 \\
\hline Sweet Bakery Products & 723 & 16 & 39.6 & 1.6 & 2.0 & 0.1 & 723 & 15 & 37.0 & 1.5 & 1.9 & 0.1 & 262 & -2.6 & 0.4 & -0.1 & 0.0 \\
\hline Mixed Dishes_-Pizza & 247 & 17 & 38.1 & 4.3 & 1.9 & 0.2 & 247 & 20 & 31.8 & 3.6 & 1.6 & 0.2 & 247 & -6.2 & 0.8 & -0.3 & 0.0 \\
\hline Ready-to-Eat Cereals & 695 & 18 & 37.0 & 2.6 & 1.9 & 0.1 & 695 & 16 & 36.9 & 2.6 & 1.9 & 0.1 & 12 & -0.1 & 0.1 & 0.0 & 0.0 \\
\hline Mixed Dishes-M/P/F & 189 & 19 & 33.1 & 5.6 & 1.7 & 0.3 & 189 & 19 & 32.1 & 5.5 & 1.6 & 0.3 & 69 & -1.1 & 0.3 & -0.1 & 0.0 \\
\hline Other Desserts & 416 & 20 & 31.3 & 3.8 & 1.6 & 0.2 & 416 & 25 & 18.9 & 2.4 & 1.0 & 0.1 & 291 & -12.4 & 1.6 & -0.6 & 0.1 \\
\hline Mixed Dishes-Sandwiches & 208 & 21 & 29.6 & 3.4 & 1.5 & 0.2 & 208 & 22 & 28.6 & 3.3 & 1.4 & 0.2 & 67 & -1.0 & 0.2 & -0.1 & 0.0 \\
\hline Mixed Dishes—Soups & 227 & 22 & 29.4 & 5.9 & 1.5 & 0.3 & 227 & 21 & 29.2 & 5.8 & 1.5 & 0.3 & 7 & -0.2 & 0.1 & 0.0 & 0.0 \\
\hline Eggs & 332 & 23 & 24.3 & 2.4 & 1.2 & 0.1 & 332 & 24 & 20.0 & 2.0 & 1.0 & 0.1 & 234 & -4.2 & 0.6 & -0.2 & 0.0 \\
\hline Meats & 220 & 24 & 23.5 & 2.7 & 1.2 & 0.1 & 220 & 23 & 23.5 & 2.7 & 1.2 & 0.1 & 5 & 0.0 & 0.0 & 0.0 & 0.0 \\
\hline Quick Bread and Bread Products & 293 & 25 & 20.5 & 3.2 & 1.0 & 0.2 & 293 & 28 & 15.4 & 2.4 & 0.8 & 0.1 & 274 & -5.1 & 0.9 & -0.3 & 0.1 \\
\hline
\end{tabular}


Table 5. Cont

\begin{tabular}{|c|c|c|c|c|c|c|c|c|c|c|c|c|c|c|c|c|c|}
\hline \multicolumn{18}{|c|}{ Mean Potassium Intake (mg) of Children 6-11 Years of Age $(n=2193)$} \\
\hline \multirow{2}{*}{$\begin{array}{c}\text { WWEIA Food Group } \\
\text { Sub Group Description }\end{array}$} & \multicolumn{6}{|c|}{ Specific Food Group Intake } & \multicolumn{6}{|c|}{ Adjusted Intake } & \multicolumn{5}{|c|}{ Delta Intake } \\
\hline & Cons & Rank & Mean & SE & Pct & SE & Cons & Rank & Mean & SE & Pct & SE & Cons & Mean & SE & Pct & SE \\
\hline Milk & 1274 & 1 & 283.4 & 10.5 & 12.9 & 0.5 & 1987 & 1 & 343.5 & 10.9 & 15.6 & 0.5 & 1646 & 60.0 & 2.8 & 2.7 & 0.1 \\
\hline Fruits & 1153 & 2 & 160.3 & 7.5 & 7.3 & 0.3 & 1153 & 2 & 160.3 & 7.5 & 7.3 & 0.3 & 6 & -0.1 & 0.0 & 0.0 & 0.0 \\
\hline Flavored Milk & 555 & 3 & 124.9 & 11.2 & 5.7 & 0.5 & 555 & 3 & 124.9 & 11.2 & 5.7 & 0.5 & 0 & 0.0 & 0.0 & 0.0 & 0.0 \\
\hline $100 \%$ Juice & 759 & 4 & 117.5 & 10.1 & 5.3 & 0.4 & 759 & 4 & 117.5 & 10.1 & 5.3 & 0.4 & 0 & 0.0 & 0.0 & 0.0 & 0.0 \\
\hline White Potatoes & 610 & 5 & 101.3 & 8.0 & 4.6 & 0.4 & 610 & 5 & 98.7 & 7.6 & 4.5 & 0.3 & 105 & -2.6 & 0.7 & -0.1 & 0.0 \\
\hline Mixed Dishes-Pizza & 548 & 6 & 96.1 & 9.5 & 4.4 & 0.4 & 548 & 8 & 82.1 & 8.1 & 3.7 & 0.4 & 547 & -14.0 & 1.6 & -0.6 & 0.1 \\
\hline Mixed Dishes-Grain-based & 606 & 7 & 90.0 & 6.8 & 4.1 & 0.3 & 606 & 9 & 80.7 & 6.4 & 3.7 & 0.3 & 324 & -9.2 & 1.3 & -0.4 & 0.1 \\
\hline Mixed Dishes-Mexican & 362 & 8 & 88.0 & 9.9 & 4.0 & 0.4 & 362 & 10 & 79.6 & 9.1 & 3.6 & 0.4 & 347 & -8.4 & 1.0 & -0.4 & 0.0 \\
\hline Savory Snacks & 1048 & 9 & 88.0 & 5.8 & 4.0 & 0.3 & 1048 & 6 & 87.8 & 5.8 & 4.0 & 0.3 & 232 & -0.2 & 0.0 & 0.0 & 0.0 \\
\hline Poultry & 711 & 10 & 85.6 & 5.4 & 3.9 & 0.2 & 711 & 7 & 85.4 & 5.4 & 3.9 & 0.2 & 124 & -0.3 & 0.1 & 0.0 & 0.0 \\
\hline Sweetened Beverages & 1467 & 11 & 77.6 & 4.8 & 3.5 & 0.2 & 1467 & 12 & 74.4 & 4.0 & 3.4 & 0.2 & 45 & -3.2 & 1.5 & -0.2 & 0.1 \\
\hline Vegetables, excluding Potatoes & 792 & 12 & 75.4 & 6.7 & 3.4 & 0.3 & 792 & 11 & 74.8 & 6.6 & 3.4 & 0.3 & 40 & -0.6 & 0.2 & 0.0 & 0.0 \\
\hline Mixed Dishes-M/P/F & 281 & 13 & 67.1 & 13.5 & 3.1 & 0.6 & 281 & 13 & 64.5 & 13.2 & 2.9 & 0.6 & 123 & -2.6 & 0.7 & -0.1 & 0.0 \\
\hline Cured Meats/Poultry & 687 & 14 & 63.0 & 3.9 & 2.9 & 0.2 & 687 & 14 & 62.9 & 3.9 & 2.9 & 0.2 & 1 & 0.0 & 0.0 & 0.0 & 0.0 \\
\hline Breads, Rolls, Tortillas & 1205 & 15 & 62.6 & 3.5 & 2.9 & 0.2 & 1205 & 15 & 62.0 & 3.4 & 2.8 & 0.2 & 85 & -0.6 & 0.2 & 0.0 & 0.0 \\
\hline Mixed Dishes-Sandwiches & 436 & 16 & 62.3 & 6.9 & 2.8 & 0.3 & 436 & 16 & 59.7 & 6.8 & 2.7 & 0.3 & 170 & -2.6 & 0.4 & -0.1 & 0.0 \\
\hline Other Desserts & 620 & 17 & 58.5 & 4.9 & 2.7 & 0.2 & 620 & 23 & 35.1 & 3.6 & 1.6 & 0.2 & 478 & -23.4 & 1.7 & -1.1 & 0.1 \\
\hline Sweet Bakery Products & 1034 & 18 & 56.8 & 3.0 & 2.6 & 0.1 & 1034 & 17 & 52.5 & 2.8 & 2.4 & 0.1 & 455 & -4.3 & 0.6 & -0.2 & 0.0 \\
\hline Plant-Based Protein Foods & 475 & 19 & 52.0 & 5.0 & 2.4 & 0.2 & 475 & 18 & 52.0 & 5.0 & 2.4 & 0.2 & 1 & 0.0 & 0.0 & 0.0 & 0.0 \\
\hline Meats & 388 & 20 & 47.1 & 4.7 & 2.1 & 0.2 & 388 & 20 & 47.0 & 4.7 & 2.1 & 0.2 & 5 & 0.0 & 0.0 & 0.0 & 0.0 \\
\hline Ready-to-Eat Cereals & 861 & 21 & 38.3 & 2.3 & 1.7 & 0.1 & 861 & 21 & 38.2 & 2.3 & 1.7 & 0.10 & 24 & -0.2 & 0.1 & 0.0 & 0.0 \\
\hline Mixed Dishes-Soups & 253 & 22 & 38.0 & 6.3 & 1.7 & 0.3 & 253 & 22 & 36.6 & 5.6 & 1.7 & 0.3 & 6 & -1.4 & 1.4 & -0.1 & 0.1 \\
\hline Condiments and Sauces & 867 & 23 & 31.5 & 3.5 & 1.4 & 0.2 & 867 & 25 & 30.9 & 3.4 & 1.4 & 0.2 & 32 & -0.6 & 0.2 & 0.0 & 0.0 \\
\hline Yogurt & 206 & 24 & 29.2 & $>3.4$ & 1.3 & 0.2 & 281 & 24 & 34.5 & 3.6 & 1.6 & 0.2 & 93 & 5.3 & 1.4 & 0.2 & 0.1 \\
\hline Quick Breads and Bread Products & 475 & 25 & 27.9 & 1.5 & 1.2 & 0.1 & 475 & 26 & 20.7 & 1.2 & 0.9 & 0.1 & 445 & -7.3 & 0.6 & -0.3 & 0.0 \\
\hline
\end{tabular}


Table 5. Cont

\begin{tabular}{|c|c|c|c|c|c|c|c|c|c|c|c|c|c|c|c|c|c|}
\hline \multicolumn{18}{|c|}{ Mean Potassium Intake $(\mathrm{mg})$ of Children 12-18 Years of Age $(n=2172)$} \\
\hline \multirow{2}{*}{$\begin{array}{c}\text { WWEIA Food Group } \\
\text { Sub Group Description }\end{array}$} & \multicolumn{6}{|c|}{ Specific Food Group Intake } & \multicolumn{6}{|c|}{ Adjusted Intake } & \multicolumn{5}{|c|}{ Delta Intake } \\
\hline & Cons & Rank & Mean & SE & Pct & SE & Cons & Rank & Mean & SE & Pct & SE & Cons & Mean & SE & Pct & SE \\
\hline White Potatoes & 580 & 2 & 145.2 & 11.5 & 6.3 & 0.4 & 580 & 2 & 140.9 & 10.9 & 6.1 & 0.4 & 121 & -4.3 & 0.8 & -0.2 & 0.0 \\
\hline Fruits & 834 & 3 & 128.8 & 9.7 & 5.6 & 0.4 & 834 & 3 & 128.8 & 9.7 & 5.6 & 0.4 & 3 & 0.0 & 0.0 & 0.0 & 0.0 \\
\hline 100\% Juice & 487 & 4 & 107.1 & 9.8 & 4.6 & 0.4 & 487 & 4 & 107.1 & 9.8 & 4.6 & 0.4 & 0 & 0.0 & 0.0 & 0.0 & 0. \\
\hline Poultry & 652 & 5 & 103.8 & 10.1 & 4.5 & 0.4 & 652 & 5 & 103.5 & 10.0 & 4.5 & 0.4 & 130 & -0.3 & 0.1 & 0.0 & 0.00 \\
\hline Mixed Dishes_-Pizza & 486 & 7 & 96.5 & 10.0 & 4.2 & 0.4 & 486 & 11 & 82.3 & 8.5 & 3.6 & 0.4 & 486 & -14.2 & 1.5 & -0.6 & 0.1 \\
\hline Mixed Dishes_-Grain-based & 464 & 8 & 96.1 & 7.9 & 4.2 & 0.3 & 464 & 9 & 86.4 & 7.5 & 3.7 & 0.3 & 244 & -9.7 & 1. & -0.4 & 0.1 \\
\hline Vegetables, excluding Potatoes & 803 & 9 & 95.4 & 8.6 & 4.1 & 0.3 & 803 & 6 & 94.8 & 8.6 & 4.1 & 0.3 & 28 & -0.6 & 0.23 & 0.0 & 0.0 \\
\hline Savory Snacks & 942 & 10 & 92.5 & 7.1 & 4.0 & 0.3 & 942 & 7 & 92.2 & 7.1 & 4.0 & 0.3 & 233 & -0.3 & 0.1 & 0.0 & 0.0 \\
\hline Meats & 424 & 11 & 85.8 & 7.7 & 3.7 & 0.3 & 424 & 10 & 85.7 & 7.7 & 3.7 & 0.3 & 14 & -0.1 & 0.0 & 0.0 & 0.0 \\
\hline Mixed Dishes-Sandwiches & 374 & 12 & 74.6 & 7.5 & 3.2 & 0.3 & 374 & 13 & 70.8 & 6.9 & 3.1 & 0.3 & 174 & -3.9 & 0.6 & -0.2 & 0.0 \\
\hline Cured Meats/Poultry & 585 & 13 & 72.1 & 5.7 & 3.1 & 0.2 & 585 & 12 & 72.0 & 5.7 & 3.1 & 0.2 & 2 & -0.1 & 0.1 & 0.0 & 0.0 \\
\hline Mixed Dishes-M/P/F & 288 & 14 & 71.2 & 9.3 & 3.1 & 0.4 & 288 & 14 & 67.8 & 9.3 & 2.9 & 0.4 & 100 & -3.4 & 0.7 & -0.2 & 0.0 \\
\hline Sweetened Beverages & 1404 & 15 & 67.1 & 5.4 & 2.9 & 0.3 & 1404 & 15 & 64.0 & 4.8 & 2.8 & 0.2 & 40 & -3.0 & 0.8 & -0.1 & 0.0 \\
\hline Plant-Based Protein Foods & 344 & 17 & 59.5 & 9.8 & 2.6 & 0.4 & 344 & 17 & 59.5 & 9.8 & 2.6 & 0.4 & 1 & 0.0 & 0.0 & 0.0 & 0.0 \\
\hline Flavored Milk & 225 & 18 & 58.3 & 7.1 & 2.5 & 0.3 & 225 & 18 & 58.3 & 7.1 & 2.5 & 0.3 & 0 & 0.0 & 0.0 & 0.0 & 0.0 \\
\hline Coffee and Tea & 505 & 19 & 52.2 & 3.4 & 2.3 & 0.2 & 505 & 23 & 44.9 & 3.6 & 1.9 & 0.2 & 60 & -7.3 & 1.2 & -0.3 & 0.1 \\
\hline Sweet Bakery Products & 833 & 20 & 50.7 & 4.4 & 2.2 & 0.2 & 833 & 20 & 46.9 & 4.1 & 2.0 & 0.2 & 344 & -3.8 & 0.5 & -0.2 & 0.0 \\
\hline Other Desserts & 377 & 21 & 48.9 & 6.1 & 2.1 & 0.3 & 377 & 26 & 26.1 & 2.7 & 1.1 & 0.1 & 339 & -22.7 & 3.8 & -1.0 & 0.2 \\
\hline Condiments and Sauces & 889 & 22 & 47.1 & 4.6 & 2.0 & 0.2 & 889 & 21 & 46.1 & 4.6 & 2.0 & 0.2 & 41 & -1.0 & 0.4 & 0.0 & 0.0 \\
\hline Mixed Dishes-Asian & 174 & 23 & 45.0 & 10.8 & 2.0 & 0.5 & 174 & 22 & 45.0 & 10.8 & 2.0 & 0.5 & 11 & 0.0 & 0.0 & 0.0 & 0.0 \\
\hline Ready-to-Eat Cereals & 582 & 24 & 39.1 & 3.7 & 1.7 & 0.2 & 582 & 24 & 39.0 & 3.7 & 1.7 & 0.2 & 18 & -0.1 & 0.04 & 0.00 & 0.0 \\
\hline Mixed Dishes-Soups & 237 & 25 & 30.6 & 4.2 & 1.3 & 0.2 & 237 & 25 & 29.7 & 4.0 & 1.3 & 0.2 & 10 & -0.9 & 0.35 & 0.0 & 0.0 \\
\hline Eggs & 333 & 26 & 24.9 & 2.0 & 1.1 & 0.1 & 333 & 27 & 21.2 & 1.7 & 0.9 & 0.1 & 211 & -3.8 & 0.38 & -0.2 & 0.0 \\
\hline
\end{tabular}

${ }^{1}$ To a $1 \%$ contribution of daily intake of potassium; ${ }^{2}$ Nutrients from milk, cheese, and yogurt for non-dairy foods are added to the nutrients in the milk, cheese, and yogurt food categories, respectively. For non-dairy foods the nutrients displayed are only for the milk, cheese, and yogurt in the non-dairy food. Abbreviations: Cons = consumers, $\mathrm{M} / \mathrm{P} / \mathrm{F}=\mathrm{meat} / \mathrm{poultry} /$ fish; $\mathrm{SE}=$ standard error; $\mathrm{Pct}=$ percent contribution to energy intake or specific nutrient intake, as appropriate. 


\subsection{Contribution of Foods to Percent Added Sugars Intake}

Total mean daily added sugars intake was $12.0 \pm 0.3$ teaspoon equivalents (tsp eq) (15.6\% of total energy); $18.2 \pm 0.4$ tsp eq (18.6\% energy); and $20.2 \pm 0.5$ tsp eq (19.6\% energy) for children $2-5,6-11$, and $12-18$ years, respectively. Table 6 shows the food sources contributing at least $1 \%$ of added sugars intake. For SFG intake, 15, 14, and 11 food groups contributed to at least $1 \%$ of added sugars intake for the three age groups, respectively. For the youngest group of children, the top three SFG and adjusted food intakes were sweetened beverages ( $3 \mathrm{tsp}$ eq; $25.3 \%$; 0.0 tsp eq), sweet bakery products (1.9 tsp eq; $16 \% ; 0.0$ tsp eq), and other desserts ( 0.9 tsp eq; $7.5 \% ; 0.0$ tsp eq). For children $6-11$ years, the top three SFG and adjusted food intakes were sweetened beverages (5.8 tsp eq; $32.1 \%$; 0.0 tsp eq (delta values)), sweet bakery products ( 2.8 tsp eq; $15.3 \% ; 0.0$ tsp eq), and candy (1.5 tsp eq; $8.2 \% ; 0.0$ tsp eq). For children 12-18 years, the top three food groups in the SFG and adjusted intakes were sweetened beverages ( 8.6 tsp eq; $42.5 \% ; 0.0$ tsp eq), sweet bakery products ( 2.4 tsp eq; $11.8 \% ; 0.0$ tsp eq), and coffee and tea (1.7 tsp eq; $1.7 \% ; 0.0$ tsp eq).

\subsection{Contribution of Foods to Percent Saturated Fatty Acids (SFA) Intake}

Total mean daily SFA intake was $20.1 \pm 0.6 \mathrm{~g}(11.8 \%$ total energy); $26.0 \pm 0.5 \mathrm{~g}(12 \%$ energy); and $26.0 \pm 0.5 \mathrm{~g}$ (11.4\% energy) for $2-5,6-11$, and $12-18$ years, respectively. Table 7 shows the food sources contributing at least $1 \%$ of SFA intake. There were 23,21 , and 24 food sources that contributed at least $1 \%$ of SFA consumed by children $2-5,6-11$, and $12-18$ years, respectively. Using SFG data for children $2-5$ years, milk ( $3.4 \mathrm{~g} ; 16.7 \%)$, sweet bakery products $(1.8 \mathrm{~g} ; 8.8 \%)$ and cheese $(1.7 \mathrm{~g} ; 8.2 \%)$ were the top sources of dietary SFA. Using adjusted data, the top ranked contributors to SFA intake in children $2-5$ years were milk ( $3.7 \mathrm{~g} ; 18.4 \% ;+0.3 \mathrm{~g}$ (delta value)), cheese $(3.3 \mathrm{~g} ; 16.4 \% ; 0.0 \mathrm{~g})$, and sweet bakery products $(1.8 \mathrm{~g} ; 8.7 \% ;+1.6 \mathrm{~g})$.

For children $6-11$ years, sweet bakery products $(2.6 \mathrm{~g} ; 10 \%)$, pizza $(2.3 \mathrm{~g} ; 9 \%)$, and milk ( $2.2 \mathrm{~g}$; $8.3 \%$ ) were the top three sources of SFA. Using adjusted data, cheese was the single highest contributor of SFA to the diet $(4.7 \mathrm{~g} ; 8.3 \% ;+3.2 \mathrm{~g})$, followed by milk $(2.6 \mathrm{~g} ; 10.1 \%$; $+0.5 \mathrm{~g})$, and sweet bakery products $(2.6 \mathrm{~g} ; 9.9 \% ; 0.0 \mathrm{~g})$. Using these adjusted data, pizza dropped to 11 th $(0.8 \mathrm{~g} ; 3.2 \%)$.

For children $12-18$ years, pizza ( $2.4 \mathrm{~g} ; 9.1 \%)$, sweet bakery products $(2.2 \mathrm{~g} ; 8.6 \%)$, and milk $(2.1 \mathrm{~g}$; $8.1 \%$ ) were the top food sources contributing to SFA intake. Using adjusted data, cheese ranked first $(5.1 \mathrm{~g} ; 19.7 \% ;+3.4 \mathrm{~g})$, followed by milk $(2.6 \mathrm{~g} ; 10 \% ;+0.5 \mathrm{~g})$, and sweet bakery products $(2.2 \mathrm{~g} ; 8.5 \%$; $0.0 \mathrm{~g}$ ). 
Table 6. Food/food group sources ${ }^{1}$ of mean added sugars (tsp eq) intake among US children aged 2-18 years $(N=5876)$ : National Health and Nutrition Examination Survey 2011-2014.

\begin{tabular}{|c|c|c|c|c|c|c|c|c|c|c|c|c|c|c|c|c|c|}
\hline \multicolumn{18}{|c|}{ Mean Added Sugars Intake (tsp eq) of Children 2-5 Years of Age $(n=1511)$} \\
\hline & \multicolumn{6}{|c|}{ Specific Food Group Intake } & \multicolumn{6}{|c|}{ Adjusted Intake ${ }^{2}$} & \multicolumn{5}{|c|}{ Delta Intake } \\
\hline Sub Group Description & Cons & Rank & Mean & SE & Pct & SE & Cons & Rank & Mean & SE & Pct & SE & Cons & Mean & SE & Pct & SE \\
\hline Sweetened Beverages & 787 & 1 & 3.0 & 0.2 & 25.3 & 1.4 & 787 & 1 & 3.0 & 0.2 & 25.2 & 1.4 & 23 & 0.0 & 0.0 & -0.1 & 0.0 \\
\hline Sweet Bakery Products & 723 & 2 & 1.9 & 0.1 & 16.0 & 0.7 & 723 & 2 & 1.9 & 0.1 & 16.0 & 0.7 & 262 & 0.0 & 0.0 & 0.0 & 0.0 \\
\hline Other Desserts & 416 & 3 & 0.9 & 0.1 & 7.5 & 0.9 & 416 & 3 & 0.9 & 0.1 & 7.4 & 0.9 & 291 & 0.0 & 0.0 & -0.1 & 0.1 \\
\hline Ready-to-Eat Cereals & 695 & 4 & 0.9 & 0.1 & 7.2 & 0.5 & 695 & 4 & 0.9 & 0.1 & 7.2 & 0.5 & 12 & 0.0 & 0.0 & 0.0 & 0.0 \\
\hline Candy & 507 & 5 & 0.8 & 0.1 & 6.9 & 0.4 & 507 & 5 & 0.8 & 0.1 & 6.9 & 0.4 & 181 & 0.0 & 0.0 & 0.0 & 0.0 \\
\hline Flavored Milk & 253 & 6 & 0.8 & 0.1 & 6.7 & 0.8 & 253 & 6 & 0.8 & 0.1 & 6.7 & 0.8 & 0 & 0.0 & 0.0 & 0.0 & 0.0 \\
\hline Sugars & 440 & 7 & 0.7 & 0.1 & 6.2 & 1.0 & 440 & 7 & 0.7 & 0.1 & 6.2 & 1.0 & 21 & 0.0 & 0.0 & 0.0 & 0.0 \\
\hline Yogurt & 231 & 8 & 0.6 & 0.1 & 5.1 & 0.7 & 278 & 8 & 0.6 & 0.1 & 5.3 & 0.7 & 60 & 0.0 & 0.0 & 0.2 & 0.2 \\
\hline Quick Breads and Bread Products & 293 & 9 & 0.4 & 0.1 & 3.0 & 0.7 & 293 & 9 & 0.4 & 0.1 & 3.0 & 0.7 & 274 & 0.0 & 0.0 & 0.0 & 0.0 \\
\hline Breads, Rolls, Tortillas & 818 & 10 & 0.3 & 0.0 & 2.1 & 0.2 & 818 & 10 & 0.3 & 0.0 & 2.1 & 0.2 & 30 & 0.0 & 0.0 & 0.0 & 0.0 \\
\hline Dairy Drinks and Substitutes & 70 & 11 & 0.2 & 0.0 & 1.5 & 0.3 & 70 & 11 & 0.2 & 0.0 & 1.5 & 0.3 & 0 & 0.0 & 0.0 & 0.0 & 0.0 \\
\hline Fruit & 945 & 12 & 0.2 & 0.0 & 1.5 & 0.2 & 945 & 12 & 0.2 & 0.0 & 1.5 & 0.2 & 2 & 0.0 & 0.0 & 0.0 & 0.0 \\
\hline Coffee and Tea & 130 & 13 & 0.2 & 0.0 & 1.5 & 0.2 & 130 & 13 & 0.2 & 0.0 & 1.5 & 0.2 & 6 & 0.0 & 0.0 & 0.0 & 0.0 \\
\hline Snack/Meal Bars & 81 & 14 & 0.2 & 0.0 & 1.4 & 0.2 & 81 & 14 & 0.2 & 0.0 & 1.4 & 0.2 & 30 & 0.0 & 0.0 & 0.0 & 0.0 \\
\hline Condiments and Sauces & 487 & 15 & 0.2 & 0.0 & 1.3 & 0.1 & 487 & 15 & 0.2 & 0.0 & 1.3 & 0.1 & 18 & 0.0 & 0.0 & 0.0 & 0.0 \\
\hline \multicolumn{18}{|c|}{ Mean Added Sugars Intake (tsp eq) of Children 6-11 Years of Age $(n=2193)$} \\
\hline & \multicolumn{6}{|c|}{ Specific Food Group Intake } & \multicolumn{6}{|c|}{ Adjusted Intake $^{2}$} & \multicolumn{5}{|c|}{ Delta Intake } \\
\hline Sub Group Description & Cons & Rank & Mean & SE & Pct & SE & Cons & Rank & Mean & SE & Pct & SE & Cons & Mean & SE & Pct & SE \\
\hline Sweetened Beverages & 1467 & 1 & 5.8 & 0.2 & 32.1 & 1.06 & 1467 & 1 & 5.82 & 0.2 & 31.7 & 1.1 & 45 & 0.0 & 0.0 & -0.1 & 0.1 \\
\hline Sweet Bakery Products & 1034 & 2 & 2.8 & 0.2 & 15.3 & 0.8 & 1034 & 2 & 2.8 & 0.2 & 15.3 & 0.8 & 455 & 0.0 & 0.0 & 0.0 & 0.0 \\
\hline Candy & 764 & 3 & 1.5 & 0.2 & 8.2 & 0.9 & 764 & 3 & 1.5 & 0.2 & 8.2 & 0.9 & 306 & 0.0 & 0.0 & 0.0 & 0.0 \\
\hline Other Desserts & 620 & 4 & 1.4 & 0.2 & 7.8 & 0.8 & 620 & 4 & 1.4 & 0.2 & 7.6 & 0.8 & 478 & 0.0 & 0.0 & -0.2 & 0.1 \\
\hline Ready-to-Eat Cereals & 861 & 5 & 1.1 & 0.1 & 6.2 & 0.5 & 861 & 5 & 1.1 & 0.1 & 6.2 & 0.5 & 24 & 0.0 & 0.0 & 0.0 & 0.00 \\
\hline Sugars & 609 & 6 & 0.9 & 0.1 & 5.1 & 0.4 & 609 & 6 & 0.9 & 0.1 & 5.1 & 0.4 & 42 & 0.0 & 0.0 & 0.0 & 0.0 \\
\hline Flavored Milk & 555 & 7 & 0.9 & 0.1 & 4.9 & 0.4 & 555 & 7 & 0.9 & 0.1 & 4.9 & 0.4 & 0 & 0.0 & 0.0 & 0.0 & 0.0 \\
\hline Coffee and Tea & 285 & 8 & 0.7 & 0.1 & 3.8 & 0.6 & 285 & 8 & 0.7 & 0.1 & 3.8 & 0.6 & 16 & 0.0 & 0.0 & 0.0 & 0.0 \\
\hline Quick Breads and Bread Products & 475 & 9 & 0.5 & 0.0 & 2.6 & 0.2 & 475 & 9 & 0.5 & 0.0 & 2.6 & 0.2 & 445 & 0.0 & 0.0 & 0.0 & 0.0 \\
\hline Breads, Rolls, Tortillas & 1205 & 10 & 0.4 & 0.0 & 2.0 & 0.1 & 1205 & 11 & 0.4 & 0.0 & 2.0 & 0.1 & 85 & 0.0 & 0.0 & 0.0 & 0.0 \\
\hline Yogurt & 206 & 11 & 0.3 & 0.0 & 1.7 & 0.2 & 281 & 10 & 0.4 & 0.0 & 2.1 & 0.2 & 93 & 0.1 & 0.0 & 0.4 & 0.1 \\
\hline Condiments and Sauces & 867 & 12 & 0.3 & 0.0 & 1.4 & 0.2 & 867 & 12 & 0.3 & 0.0 & 1.4 & 0.2 & 32 & 0.0 & 0.0 & 0.0 & 0.0 \\
\hline Dairy Drinks and Substitutes & 81 & 13 & 0.2 & 0.1 & 1.3 & 0.3 & 81 & 13 & 0.2 & 0.0 & 1.3 & 0.3 & 0 & 0.0 & 0.0 & 0.0 & 0.0 \\
\hline Mixed Dishes Sandwiches & 436 & 14 & 0.2 & 0.0 & 1.2 & 0.1 & 436 & 14 & 0.2 & 0.0 & 1.2 & 0.1 & 170 & 0.0 & 0.0 & 0.0 & 0.0 \\
\hline
\end{tabular}


Table 6. Cont.

\begin{tabular}{|c|c|c|c|c|c|c|c|c|c|c|c|c|c|c|c|c|c|}
\hline \multirow[b]{3}{*}{ Sub Group Description } & \multicolumn{12}{|c|}{ Mean Added Sugars Intake (tsp eq) of Children 12-18 Years of Age $(n=2172)$} & \multirow{2}{*}{\multicolumn{5}{|c|}{ Delta Intake }} \\
\hline & \multicolumn{6}{|c|}{ Specific Food Group Intake } & \multicolumn{5}{|c|}{ Adjusted Intake } & \multirow[b]{2}{*}{ SE } & & & & & \\
\hline & Cons & Rank & Mean & SE & Pct & SE & Cons & Rank & Mean & SE & Pct & & Cons & Mean & SE & Pct & SE \\
\hline Sweetened Beverages & 1404 & 1 & 8.6 & 0.3 & 42.5 & 1.3 & 1404 & 1 & 8.6 & 0.3 & 42.4 & 1.3 & 40 & 0.0 & 0.0 & -0.1 & 0.0 \\
\hline Sweet Bakery Products & 833 & 2 & 2.4 & 0.2 & 11.8 & 0.8 & 833 & 2 & 2.4 & 0.2 & 11.8 & 0.8 & 344 & 0.0 & 0.0 & 0.0 & 0.0 \\
\hline Coffee and Tea & 505 & 3 & 1.7 & 0.3 & 8.3 & 1.4 & 505 & 3 & 1.7 & 0.3 & 8.3 & 1.4 & 60 & 0.0 & 0.0 & 0.0 & 0.0 \\
\hline Candy & 600 & 4 & 1.1 & 0.2 & 5.6 & 0.7 & 600 & 4 & 1.1 & 0.2 & 5.6 & 0.7 & 273 & 0.0 & 0.0 & 0.0 & 0.0 \\
\hline Ready-to-Eat Cereals & 582 & 5 & 1.0 & 0.1 & 5.0 & 0.4 & 582 & 5 & 1.0 & 0.1 & 5.0 & 0.4 & 18 & 0.0 & 0.0 & 0.0 & 0.0 \\
\hline Sugars & 446 & 6 & 1.0 & 0.1 & 5.0 & 0.7 & 446 & 6 & 1.0 & 0.1 & 4.9 & 0.7 & 16 & 0.0 & 0.0 & 0.0 & 0.0 \\
\hline Other Desserts & 377 & 7 & 1.0 & 0.1 & 4.9 & 0.5 & 377 & 7 & 0.9 & 0.1 & 4.6 & 0.4 & 339 & -0.1 & 0.0 & -0.3 & 0.2 \\
\hline Flavored Milk & 225 & 8 & 0.5 & 0.1 & 2.2 & 0.3 & 225 & 8 & 0.5 & 0.1 & 2.2 & 0.3 & 0 & 0.0 & 0.0 & 0.0 & 0.0 \\
\hline Breads, Rolls, Tortillas & 1119 & 9 & 0.4 & 0.0 & 1.8 & 0.1 & 1119 & 9 & 0.4 & 0.0 & 1.8 & 0.1 & 85 & 0.0 & 0.0 & 0.0 & 0.0 \\
\hline Quick Breads and Bread Products & 297 & 10 & 0.4 & 0.1 & 1.7 & 0.2 & 297 & 10 & 0.4 & 0.1 & 1.7 & 0.2 & 282 & 0.0 & 0.0 & 0.0 & 0.0 \\
\hline Dairy Drinks and Substitutes & 79 & 11 & 0.3 & 0.1 & 1.4 & 0.3 & 79 & 11 & 0.3 & 0.1 & 1.4 & 0.3 & 0 & 0.0 & 0.0 & 0.0 & 0.0 \\
\hline Snack/Meal Bars & 135 & 12 & 0.3 & 0.0 & 1.3 & 0.2 & 135 & 12 & 0.3 & 0.0 & 1.3 & 0.2 & 47 & 0.0 & 0.0 & 0.0 & 0.0 \\
\hline Condiments and Sauces & 889 & 13 & 0.2 & 0.0 & 1.2 & 0.1 & 889 & 13 & 0.2 & 0.0 & 1.2 & 0.1 & 41 & 0.0 & 0.0 & 0.0 & 0.0 \\
\hline
\end{tabular}

${ }^{1}$ To a $1 \%$ contribution of daily intake of added sugars; ${ }^{2}$ Nutrients from milk, cheese, and yogurt for non-dairy foods are added to the nutrients in the milk, cheese, and yogurt food categories, respectively. For non-dairy foods the nutrients displayed are only for the milk, cheese, and yogurt in the non-dairy food. Abbreviations: Cons $=$ consumers, $\mathrm{M} / \mathrm{P} / \mathrm{F}=$ meat/poultry / fish; SE = standard error; Pct = percent contribution to energy intake or specific nutrient intake, as appropriate. 
Table 7. Food/food group sources ${ }^{1}$ of mean saturated fatty acids $(\mathrm{g})$ intake among US children aged $2-18$ years $(N=5876)$ : National Health and Nutrition Examination Survey 2011-2014.

\begin{tabular}{|c|c|c|c|c|c|c|c|c|c|c|c|c|c|c|c|c|c|}
\hline \multicolumn{18}{|c|}{ Mean Saturated Fatty Acids Intake (g) of Children 2 to 5 Years of Age $(n=1511)$} \\
\hline \multirow{2}{*}{$\begin{array}{c}\text { WWEIA Food Group } \\
\text { Sub Group Description }\end{array}$} & \multicolumn{6}{|c|}{ Specific Food Group Intake } & \multicolumn{6}{|c|}{ Adjusted Intake $^{2}$} & \multicolumn{5}{|c|}{ Delta Intake } \\
\hline & Cons & Rank & Mean & SE & Pct & SE & Cons & Rank & Mean & SE & Pct & SE & Cons & Mean & SE & Pct & SE \\
\hline Milk & 1120 & 1 & 3.4 & 0.2 & 16.7 & 0.6 & 1415 & 1 & 3.7 & 0.2 & 18.4 & 0.6 & 1120 & 0.3 & 0.0 & 1.6 & 0.1 \\
\hline Sweet Bakery Products & 723 & 2 & 1.8 & 0.1 & 8.8 & 0.5 & 723 & 3 & 1.8 & 0.1 & 8.7 & 0.5 & 262 & 0.0 & 0.0 & -0.1 & 0.0 \\
\hline Cheese & 542 & 3 & 1.7 & 0.2 & 8.2 & 1.0 & 1047 & 2 & 3.3 & 0.3 & 16.4 & 1.0 & 820 & 1.6 & 0.1 & 8.1 & 0.4 \\
\hline Mixed Dishes-Grain-based & 466 & 4 & 1.2 & 0.1 & 5.7 & 0.5 & 466 & 5 & 0.9 & 0.1 & 4.3 & 0.4 & 266 & -0.3 & 0.0 & -1.4 & 0.1 \\
\hline Mixed Dishes-Mexican & 201 & 5 & 1.1 & 0.1 & 5.7 & 0.6 & 201 & 10 & 0.6 & 0.1 & 3.1 & 0.3 & 189 & -0.5 & 0.1 & -2.6 & 0.3 \\
\hline Cured Meats/Poultry & 459 & 6 & 1.0 & 0.1 & 4.8 & 0.5 & 459 & 4 & 1.0 & 0.1 & 4.8 & 0.5 & 2 & 0.0 & 0.0 & 0.0 & 0.0 \\
\hline Mixed Dishes-Pizza & 247 & 7 & 0.9 & 0.1 & 4.7 & 0.6 & 247 & 21 & 0.3 & 0.0 & 1.4 & 0.2 & 247 & -0.7 & 0.1 & -3.3 & 0.4 \\
\hline Flavored Milk & 253 & 8 & 0.8 & 0.1 & 3.9 & 0.5 & 253 & 6 & 0.8 & 0.1 & 3.9 & 0.5 & 0 & 0.0 & 0.0 & 0.0 & 0.0 \\
\hline Other Desserts & 416 & 9 & 0.8 & 0.1 & 3.9 & 0.5 & 416 & 7 & 0.7 & 0.1 & 3.4 & 0.4 & 291 & -0.1 & 0.0 & -0.5 & 0.1 \\
\hline Mixed Dishes-Sandwiches & 208 & 10 & 0.7 & 0.1 & 3.4 & 0.3 & 208 & 11 & 0.6 & 0.1 & 3.1 & 0.3 & 67 & -0.1 & 0.0 & -0.4 & 0.1 \\
\hline Eggs & 332 & 11 & 0.7 & 0.1 & 3.3 & 0.4 & 332 & 12 & 0.6 & 0.1 & 2.8 & 0.3 & 234 & -0.1 & 0.0 & -0.5 & 0.1 \\
\hline Fats and Oils & 460 & 12 & 0.7 & 0.1 & 3.2 & 0.3 & 460 & 8 & 0.7 & 0.1 & 3.2 & 0.3 & 31 & 0.0 & 0.0 & 0. & 0.0 \\
\hline Poultry & 526 & 13 & 0.7 & 0.1 & 3.2 & 0.3 & 526 & 9 & 0.7 & 0.1 & 3.2 & 0.3 & 71 & 0.0 & 0.0 & 0.0 & 0.0 \\
\hline Savory Snacks & 688 & 14 & 0.5 & 0.0 & 2.6 & 0.2 & 688 & 13 & 0.5 & 0.0 & 2.6 & 0.2 & 140 & 0.0 & 0.0 & -0.1 & 0.0 \\
\hline Candy & 507 & 15 & 0.5 & 0.1 & 2.4 & 0.3 & 507 & 14 & 0.5 & 0.1 & 2.3 & 0.3 & 181 & 0.0 & 0.0 & -0.1 & 0.0 \\
\hline Crackers & 353 & 16 & 0.4 & 0.0 & 1.9 & 0.2 & 353 & 16 & 0.4 & 0.0 & 1.8 & 0.2 & 86 & 0.0 & 0.0 & -0.1 & 0.0 \\
\hline Plant-Based Protein Foods & 346 & 17 & 0.4 & 0.0 & 1.9 & 0.2 & 346 & 15 & 0.4 & 0.0 & 1.9 & 0.2 & 1 & 0.0 & 0.0 & 0.0 & 0.0 \\
\hline Quick Breads and Bread Products & 293 & 18 & 0.4 & 0.1 & 1.8 & 0.3 & 293 & 18 & 0.3 & 0.0 & 1.6 & 0.2 & 274 & 0.0 & 0.0 & -0.2 & 0.0 \\
\hline Breads, Rolls, Tortillas & 818 & 19 & 0.4 & 0.1 & 1.7 & 0.2 & 818 & 17 & 0.3 & 0.1 & 1.7 & 0.2 & 30 & 0.0 & 0.0 & 0.0 & 0.0 \\
\hline Meats & 220 & 20 & 0.3 & 0.1 & 1.5 & 0.3 & 220 & 19 & 0.3 & 0.1 & 1.5 & 0.3 & 5 & 0.0 & 0.0 & 0.0 & 0.0 \\
\hline White Potatoes & 401 & 21 & 0.3 & 0.0 & 1.4 & 0.2 & 401 & 22 & 0.3 & 0.0 & 1.3 & 0.2 & 78 & 0.0 & 0.0 & -0.1 & 0.0 \\
\hline Mixed Dishes-M/P/F & 189 & 22 & 0.3 & 0.0 & 1.4 & 0.2 & 189 & 23 & 0.3 & 0.0 & 1.3 & 0.1 & 69 & 0.0 & 0.0 & -0.1 & 0.0 \\
\hline Yogurt & 231 & 23 & 0.3 & 0.0 & 1.3 & 0.2 & 278 & 20 & 0.3 & 0.0 & 1.4 & 0.2 & 60 & 0.0 & 0.0 & 0.1 & 0.0 \\
\hline
\end{tabular}


Table 7. Cont.

\begin{tabular}{|c|c|c|c|c|c|c|c|c|c|c|c|c|c|c|c|c|c|}
\hline \multicolumn{18}{|c|}{ Mean Intake of Saturated Fatty Acids Intake (g) of Children 6-11 Years of Age $(n=2193)$} \\
\hline \multirow{2}{*}{$\begin{array}{c}\text { WWEIA Food Group } \\
\text { Sub Group Description }\end{array}$} & \multicolumn{6}{|c|}{ Specific Food Group Intake } & \multicolumn{6}{|c|}{ Adjusted Intake } & \multicolumn{5}{|c|}{ Delta Intake } \\
\hline & Cons & Rank & Mean & SE & Pct & SE & Cons & Rank & Mean & SE & Pct & SE & Cons & Mean & SE & Pct & SE \\
\hline Sweet Bakery Products & 1034 & 1 & 2.6 & 0.2 & 10.0 & 0.7 & 1034 & 3 & 2.6 & 0.2 & 9.9 & 0.7 & 455 & 0.0 & 0.0 & -0.1 & 0.0 \\
\hline Mixed Dishes_-Pizza & 548 & 2 & 2.3 & 0.3 & 9.0 & 1.0 & 548 & 11 & 0.8 & 0.1 & 3.2 & 0.4 & 547 & -1.5 & 0.2 & -5.7 & 0.6 \\
\hline Milk & 1274 & 3 & 2.2 & 0.1 & 8.3 & 0.4 & 1987 & 2 & 2.6 & 0.1 & 10.1 & 0.4 & 1646 & 0.5 & 0.0 & 1.8 & 0.1 \\
\hline Mixed Dishes-Mexican & 362 & 4 & 2.0 & 0.2 & 7.9 & 0.9 & 362 & 6 & 1.2 & 0.1 & 4.5 & 0.5 & 347 & -0.9 & 0.1 & -3.4 & 0.4 \\
\hline Cheese & 723 & 5 & 1.6 & 0.1 & 6.1 & 0.5 & 1643 & 1 & 4.7 & 0.2 & 18.3 & 0.7 & 1349 & 3.2 & 0.2 & 12.1 & 0.7 \\
\hline Other Desserts & 620 & 6 & 1.5 & 0.1 & 5.9 & 0.5 & 620 & 4 & 1.4 & 0.1 & 5.2 & 0.5 & 478 & -0.2 & 0.0 & -0.7 & 0.1 \\
\hline Mixed Dishes-Grain-based & 606 & 7 & 1.5 & 0.2 & 5.7 & 0.8 & 606 & 7 & 1.1 & 0.2 & 4.1 & 0.6 & 324 & -0.4 & 0.1 & -1.6 & 0.2 \\
\hline Mixed Dishes-Sandwiches & 436 & 8 & 1.4 & 0.1 & 5.5 & 0.5 & 436 & 5 & 1.2 & 0.1 & 4.6 & 0.4 & 170 & -0.2 & 0.0 & -0.9 & 0.1 \\
\hline Fats and Oils & 740 & 9 & 1.0 & 0.1 & 4.0 & 0.4 & 740 & 8 & 1.0 & 0.1 & 4.0 & 0.4 & 62 & 0.0 & 0.0 & 0.0 & 0.0 \\
\hline Poultry & 711 & 10 & 0.9 & 0.1 & 3.4 & 0.3 & 711 & 9 & 0.9 & 0.1 & 3.4 & 0.3 & 124 & 0.0 & 0.0 & 0.0 & 0.0 \\
\hline Cured Meats/Poultry & 687 & 11 & 0.9 & 0.1 & 3.3 & 0.3 & 687 & 10 & 0.9 & 0.1 & 3.3 & 0.3 & 1 & 0.0 & 0.0 & 0.0 & 0.0 \\
\hline Savory Snacks & 1048 & 12 & 0.8 & 0.1 & 3.3 & 0.2 & 1048 & 12 & 0.8 & 0.1 & 3.2 & 0.2 & 232 & 0.0 & 0.0 & -0.1 & 0.0 \\
\hline Candy & 764 & 13 & 0.8 & 0.2 & 3.2 & 0.8 & 764 & 13 & 0.8 & 0.2 & 3.1 & 0.8 & 306 & 0.0 & 0.0 & -0.1 & 0.0 \\
\hline Flavored Milk & 555 & 14 & 0.8 & 0.1 & 2.9 & 0.3 & 555 & 14 & 0.8 & 0.1 & 2.9 & 0.3 & 0 & 0.0 & 0.0 & 0.0 & 0.0 \\
\hline Meats & 388 & 15 & 0.6 & 0.1 & 2.4 & 0.3 & 388 & 15 & 0.6 & 0.1 & 2.4 & 0.3 & 5 & 0.0 & 0.0 & 0.0 & 0.0 \\
\hline Mixed Dishes-M/P/F & 281 & 16 & 0.6 & 0.1 & 2.3 & 0.4 & 281 & 16 & 0.6 & 0.1 & 2.1 & 0.4 & 123 & -0.1 & 0.0 & -0.2 & 0.1 \\
\hline Eggs & 324 & 17 & 0.6 & 0.1 & 2.2 & 0.2 & 324 & 17 & 0.5 & 0.1 & 1.9 & 0.2 & 217 & -0.1 & 0.0 & -0.3 & 0.1 \\
\hline Quick Breads and Bread Products & 475 & 18 & 0.5 & 0.1 & 2.0 & 0.2 & 475 & 18 & 0.5 & 0.1 & 1.8 & 0.2 & 445 & -0.1 & 0.0 & -0.2 & 0.0 \\
\hline White Potatoes & 610 & 19 & 0.4 & 0.0 & 1.6 & 0.1 & 610 & 21 & 0.4 & 0.0 & 1.5 & 0.1 & 105 & 0.0 & 0.0 & -0.1 & 0.0 \\
\hline Plant-Based Protein Foods & 475 & 20 & 0.4 & 0.1 & 1.6 & 0.2 & 475 & 19 & 0.4 & 0.1 & 1.6 & 0.2 & 1 & 0.0 & 0.0 & 0.0 & 0.0 \\
\hline Breads, Rolls, Tortillas & 1205 & 21 & 0.4 & 0.0 & 1.6 & 0.1 & 1205 & 20 & 0.4 & 0.0 & 1.5 & 0.1 & 85 & 0.0 & 0.0 & -0.1 & 0.0 \\
\hline
\end{tabular}


Table 7. Cont

\begin{tabular}{|c|c|c|c|c|c|c|c|c|c|c|c|c|c|c|c|c|c|}
\hline \multicolumn{18}{|c|}{ Mean Saturated Fatty Acids (g) Intake of Children 12-18 Years of Age $(n=2172)$} \\
\hline \multirow{2}{*}{$\begin{array}{c}\text { WWEIA Food Group } \\
\text { Sub Group Description }\end{array}$} & \multicolumn{6}{|c|}{ Specific Food Group Intake } & \multicolumn{6}{|c|}{ Adjusted Intake } & \multicolumn{5}{|c|}{ Delta Intake } \\
\hline & Cons & Rank & Mean & SE & Pct & SE & Cons & Rank & Mean & SE & Pct & SE & Cons & Mean & SE & Pct & SE \\
\hline Mixed Dishes-Pizza & 486 & 1 & 2.4 & 0.2 & 9.1 & 0.9 & 486 & 12 & 0.9 & 0.1 & 3.3 & 0.3 & 486 & -1.5 & 0.2 & -5.8 & 0.6 \\
\hline Sweet Bakery Products & 833 & 2 & 2.2 & 0.2 & 8.6 & 0.6 & 833 & 3 & 2.2 & 0.2 & 8.5 & 0.6 & 344 & 0.0 & 0.0 & -0.1 & 0.0 \\
\hline Milk & 973 & 3 & 2.1 & 0.1 & 8.1 & 0.5 & 1779 & 2 & 2.6 & 0.1 & 10.0 & 0.4 & 1453 & 0.5 & 0.0 & 1.9 & 0.1 \\
\hline Mixed Dishes-Mexican & 329 & 4 & 2.1 & 0.2 & 7.9 & 0.9 & 329 & 5 & 1.2 & 0.1 & 4.5 & 0.5 & 313 & -0.9 & 0.1 & -3.4 & 0.5 \\
\hline Cheese & 687 & 5 & 1.7 & 0.1 & 6.7 & 0.4 & 1565 & 1 & 5.1 & 0.2 & 19.7 & 0.7 & 1276 & 3.4 & 0.2 & 13.1 & 0.7 \\
\hline Mixed Dishes-Sandwiches & 374 & 6 & 1.6 & 0.2 & 6.1 & 0.7 & 374 & 4 & 1.3 & 0.1 & 4.8 & 0.5 & 174 & -0.3 & 0.1 & -1.3 & 0.2 \\
\hline Mixed Dishes-Grain-based & 464 & 7 & 1.4 & 0.1 & 5.5 & 0.5 & 464 & 8 & 1.0 & 0.1 & 3.9 & 0.4 & 244 & -0.4 & 0.1 & -1.6 & 0.2 \\
\hline Other Desserts & 377 & 8 & 1.2 & 0.1 & 4.6 & 0.5 & 377 & 7 & 1.0 & 0.1 & 3.9 & 0.4 & 339 & -0.2 & 0.0 & -0.6 & 0.1 \\
\hline Meats & 424 & 9 & 1.0 & 0.1 & 4.0 & 0.4 & 424 & 6 & 1.0 & 0.1 & 4.0 & 0.4 & 14 & 0.0 & 0.0 & 0.0 & 0.0 \\
\hline Poultry & 652 & 10 & 1.0 & 0.1 & 3.8 & 0.4 & 652 & 9 & 1.0 & 0.1 & 3.8 & 0.4 & 130 & 0.0 & 0.0 & 0.0 & 0.0 \\
\hline Fats and Oils & 700 & 11 & 1.0 & 0.1 & 3.8 & 0.4 & 700 & 10 & 1.0 & 0.1 & 3.8 & 0.4 & 86 & 0.0 & 0.0 & 0.0 & 0.0 \\
\hline Cured Meats/Poultry & 585 & 12 & 0.9 & 0.1 & 3.6 & 0.4 & 585 & 11 & 0.9 & 0.1 & 3.5 & 0.4 & 2 & 0.0 & 0.0 & 0.0 & 0.0 \\
\hline Savory Snacks & 942 & 13 & 0.8 & 0.1 & 3.2 & 0.4 & 942 & 13 & 0.8 & 0.1 & 3.1 & 0.4 & 233 & 0.0 & 0.0 & -0.1 & 0.0 \\
\hline Candy & 600 & 14 & 0.7 & 0.1 & 2.6 & 0.3 & 600 & 14 & 0.6 & 0.1 & 2.5 & 0.3 & 273 & 0.0 & 0.0 & -0.1 & 0.0 \\
\hline Mixed Dishes-M/P/F & 288 & 15 & 0.7 & 0.1 & 2.6 & 0.3 & 288 & 16 & 0.6 & 0.1 & 2.2 & 0.3 & 100 & -0.1 & 0.0 & -0.4 & 0.1 \\
\hline Eggs & 333 & 16 & 0.7 & 0.1 & 2.5 & 0.2 & 333 & 15 & 0.6 & 0.1 & 2.2 & 0.2 & 211 & -0.1 & 0.0 & -0.3 & 0.0 \\
\hline White Potatoes & 580 & 17 & 0.6 & 0.1 & 2.2 & 0.2 & 580 & 19 & 0.5 & 0.0 & 1.7 & 0.2 & 121 & -0.1 & 0.0 & -0.5 & 0.1 \\
\hline Breads, Rolls, Tortillas & 1119 & 18 & 0.5 & 0.0 & 2.0 & 0.1 & 1119 & 17 & 0.5 & 0.0 & 1.9 & 0.1 & 85 & 0.0 & 0.0 & -0.1 & 0.0 \\
\hline Plant-Based Protein Foods & 344 & 19 & 0.5 & 0.1 & 1.8 & 0.3 & 344 & 18 & 0.5 & 0.1 & 1.8 & 0.3 & 1 & 0.0 & 0.0 & 0.0 & 0.0 \\
\hline Quick Breads and Bread Products & 297 & 20 & 0.4 & 0.1 & 1.6 & 0.2 & 297 & 20 & 0.4 & 0.0 & 1.4 & 0.2 & 282 & 0.0 & 0.0 & -0.2 & 0.0 \\
\hline Dairy Drinks and Substitutes & 79 & 21 & 0.4 & 0.1 & 1.3 & 0.2 & 79 & 21 & 0.4 & 0.1 & 1.3 & 0.2 & 0 & 0.0 & 0.0 & 0.0 & 0.0 \\
\hline Flavored Milk & 225 & 22 & 0.3 & 0.1 & 1.3 & 0.2 & 225 & 22 & 0.3 & 0.1 & 1.3 & 0.2 & 0 & 0.0 & 0.0 & 0.0 & 0.0 \\
\hline Mixed Dishes_-Asian & 174 & 23 & 0.3 & 0.1 & 1.2 & 0.3 & 174 & 23 & 0.3 & 0.1 & 1.2 & 0.3 & 11 & 0.0 & 0.0 & 0.0 & 0.0 \\
\hline Mixed Dishes-Soups & 237 & 24 & 0.3 & 0.0 & 1.1 & 0.1 & 237 & 24 & 0.3 & 0.0 & 1.0 & 0.1 & 10 & 0.0 & 0.0 & -0.1 & 0.0 \\
\hline
\end{tabular}

${ }^{1}$ To a $1 \%$ contribution of daily intake of SFA; ${ }^{2}$ Nutrients from milk, cheese, and yogurt for non-dairy foods are added to the nutrients in the milk, cheese, and yogurt food categories, respectively. For non-dairy foods the nutrients displayed are only for the milk, cheese, and yogurt in the non-dairy food. Abbreviations: Cons $=$ consumers, $\mathrm{M} / \mathrm{P} / \mathrm{F}=\mathrm{meat} / \mathrm{poultry} /$ fish $\mathrm{SE}=$ standard error; $\mathrm{Pct}=$ percent contribution to energy intake or specific nutrient intake, as appropriate. 


\subsection{Contribution of Foods to Percent Sodium Intake}

Total daily mean intake of sodium was $2267.4 \pm 37.3 \mathrm{mg} ; 3036 \pm 40.1 \mathrm{mg}$; and $3394.8 \pm 66.6 \mathrm{mg}$ for children $2-5,6-11$, and 12-18 years, respectively. Table 8 shows the food sources contributing at least $1 \%$ of sodium intake. There were 28,28 , and 26 food sources that contributed at least $1 \%$ of sodium consumed by children $2-5,6-11$, and $12-18$ years, respectively. Using SFG data for children $2-5$ years, cured meats / poultry (183 mg; 8.1\%); grain-based mixed dishes (155 mg; 6.8\%); and bread, rolls, and tortillas ( $144 \mathrm{mg} ; 6.4 \%$ ) were the top ranked contributors of sodium to the diet. Using adjusted data, cheese was the top contributor to sodium intake (188 mg; $8.3 \% ;+88 \mathrm{mg}$ ); followed by cured meats / poultry (182 mg; $8 \% ;-0.3 \mathrm{mg}$ ); and breads, rolls, and tortillas (144 mg; $6.4 \%$; $-0.4 \mathrm{mg}$ ).

For children 6-11 years, pizza was the top SFG contributor of sodium to the diet ( $286 \mathrm{mg} ; 9.4 \%)$, followed by Mexican foods ( $215 \mathrm{mg} ; 7.1 \%$ ), and cured meats/poultry (197 mg; 6.5\%). Using adjusted data, cheese was the top contributor of sodium to the diet $(277 \mathrm{mg} ; 9.1 \%$; $+169 \mathrm{mg}$ ), followed by pizza (207 mg; 6.8\%; -79 mg), and cured meats/poultry (197 mg; 6.5\%).

Using SFG data, pizza was the top contributor of sodium to the diet (297 $\mathrm{mg} ; 8.7 \%)$, followed by Mexican foods ( $224 \mathrm{mg}$; $6.6 \%$ ), and cured meats / poultry ( $223 \mathrm{mg} ; 6.6 \%$ ) in children $12-18$ years. Using adjusted data, cheese was the top contributor of sodium to the diet $(300 \mathrm{mg} ; 8.8 \%$; $+182 \mathrm{mg}$ ), followed by cured meats/poultry (221 mg; 6.5\%; $-0.6 \mathrm{mg})$, and pizza ( $216 \mathrm{mg} ; 6.4 \% ;-80 \mathrm{mg})$. 
Table 8. Food/food group sources ${ }^{1}$ of mean sodium (mg) intake among US children aged 2-18 years $(N=5876)$ : National Health and Nutrition Examination Survey 2011-2014.

\begin{tabular}{|c|c|c|c|c|c|c|c|c|c|c|c|c|c|c|c|c|c|}
\hline \multicolumn{18}{|c|}{ Mean Sodium Intake $(\mathrm{mg})$ of Children 2-5 Years of Age $(n=1511)$} \\
\hline \multirow{2}{*}{$\begin{array}{c}\text { WWEIA Food Group } \\
\text { Sub Group Description }\end{array}$} & \multicolumn{6}{|c|}{ Actual Intake } & \multicolumn{6}{|c|}{ Adjusted Intake ${ }^{2}$} & \multicolumn{5}{|c|}{ Delta Intake } \\
\hline & Cons & Rank & Mean & SE & Pct & SE & Cons & Rank & Mean & SE & Pct & SE & Cons & Mean & SE & Pct & SE \\
\hline Cured Meats/Poultry & 459 & 1 & 182.7 & 20.3 & 8.1 & 0.8 & 459 & 2 & 182.4 & 20.2 & 8.0 & 0.8 & 2 & -0.3 & 0.3 & 0.0 & 0.0 \\
\hline Mixed Dishes-Grain-based & 466 & 2 & 155.1 & 12.3 & 6.8 & 0.5 & 466 & 4 & 140.3 & 11.8 & 6.2 & 0.5 & 266 & -14.9 & 1.4 & -0.7 & 0.1 \\
\hline Breads, Rolls, Tortillas & 818 & 3 & 144.4 & 10.6 & 6.4 & 0.5 & 818 & 3 & 144.0 & 10.5 & 6.4 & 0.5 & 30 & -0.4 & 0.2 & 0.0 & 0.0 \\
\hline Poultry & 526 & 4 & 131.3 & 11.4 & 5.8 & 0.5 & 526 & 6 & 131.2 & 11.4 & 5.8 & 0.5 & 71 & 0.0 & 0.0 & 0.0 & 0.0 \\
\hline Milk & 1120 & 5 & 120.7 & 4.4 & 5.3 & 0.2 & 1415 & 5 & 133.8 & 4.2 & 5.9 & 0.2 & 1120 & 13.2 & 0.7 & 0.6 & 0.0 \\
\hline Mixed Dishes—Pizza & 247 & 6 & 118.4 & 13.4 & 5.2 & 0.6 & 247 & 11 & 83.1 & 9.4 & 3.7 & 0.4 & 247 & -35.3 & 4.3 & -1.6 & 0.2 \\
\hline Mixed Dishes-Mexican & 201 & 7 & 116.4 & 13.3 & 5.1 & 0.6 & 201 & 9 & 88.7 & 10.2 & 3.9 & 0.5 & 189 & -27.7 & 3.5 & -1.2 & 0.2 \\
\hline Cheese & 542 & 8 & 99.5 & 11.6 & 4.4 & 0.5 & 1047 & 1 & 187.5 & 13.1 & 8.3 & 0.5 & 820 & 88.0 & 4.4 & 3.9 & 0.2 \\
\hline Mixed Dishes_-Sandwiches & 208 & 9 & 97.2 & 10.1 & 4.3 & 0.4 & 208 & 7 & 92.9 & 9.7 & 4.1 & 0.4 & 67 & -4.3 & 0.9 & -0.2 & 0.0 \\
\hline Sweet Bakery Products & 723 & 10 & 90.6 & 3.8 & 4.0 & 0.2 & 723 & 8 & 89.7 & 3.8 & 4.0 & 0.2 & 262 & -0.9 & 0.1 & 0.0 & 0.0 \\
\hline Savory Snacks & 688 & 11 & 85.8 & 11.6 & 3.8 & 0.5 & 688 & 10 & 85.3 & 11.6 & 3.8 & 0.5 & 140 & -0.5 & 0.1 & 0.0 & 0.0 \\
\hline Quick Breads and Bread Products & 293 & 12 & 74.2 & 8.3 & 3.3 & 0.4 & 293 & 13 & 72.6 & 8.1 & 3.2 & 0.4 & 274 & -1.6 & 0.3 & -0.1 & 0.0 \\
\hline Mixed Dishes-Soups & 227 & 13 & 72.9 & 10.8 & 3.2 & 0.5 & 227 & 12 & 72.7 & 10.8 & 3.2 & 0.5 & 7 & -0.2 & 0.1 & 0.0 & 0.0 \\
\hline Ready-to-Eat Cereals & 695 & 14 & 67.7 & 3.5 & 3.0 & 0.2 & 695 & 14 & 67.7 & 3.5 & 3.0 & 0.2 & 12 & 0.0 & 0.0 & 0.0 & 0.0 \\
\hline Crackers & 353 & 15 & 65.3 & 6.2 & 2.9 & 0.3 & 353 & 15 & 64.3 & 6.1 & 2.8 & 0.3 & 86 & -1.0 & 0.2 & 0.0 & 0.0 \\
\hline Mixed Dishes-M/P/F & 189 & 16 & 63.2 & 10.7 & 2.8 & 0.5 & 189 & 16 & 62.3 & 10.8 & 2.8 & 0.5 & 69 & -0.9 & 0.3 & 0.0 & 0.0 \\
\hline Eggs & 332 & 17 & 62.9 & 6.4 & 2.8 & 0.3 & 332 & 18 & 58.5 & 5.8 & 2.6 & 0.3 & 234 & -4.4 & 0.9 & -0.20 & 0.0 \\
\hline Condiments and Sauces & 487 & 18 & 61.0 & 5.8 & 2.7 & 0.3 & 487 & 17 & 60.7 & 5.7 & 2.7 & 0.3 & 18 & -0.3 & 0.1 & 0.0 & 0.0 \\
\hline Vegetables, excluding Potatoes & 560 & 19 & 55.7 & 4.5 & 2.5 & 0.2 & 560 & 19 & 55.1 & 4.6 & 2.4 & 0.2 & 22 & -0.6 & 0.4 & 0.0 & 0.0 \\
\hline White Potatoes & 401 & 20 & 45.7 & 4.1 & 2.0 & 0.2 & 401 & 20 & 44.9 & 4.1 & 2.0 & 0.2 & 78 & -0.8 & 0.2 & 0.0 & 0.0 \\
\hline Cooked Grains & 299 & 21 & 44.5 & 4.9 & 2.0 & 0.2 & 299 & 21 & 44.5 & 4.9 & 2.0 & 0.2 & 0 & 0.0 & 0.0 & 0.0 & 0.0 \\
\hline Flavored Milk & 253 & 22 & 38.1 & 4.8 & 1.7 & 0.2 & 253 & 22 & 38.1 & 4.8 & 1.7 & 0.2 & 0 & 0.0 & 0.0 & 0.0 & 0.0 \\
\hline Plant-Based Protein Foods & 346 & 23 & 31.9 & 3.5 & 1.4 & 0.2 & 346 & 23 & 31.9 & 3.5 & 1.4 & 0.2 & 1 & 0.0 & 0.0 & 0.0 & 0.0 \\
\hline Mixed Dishes-Asian & 89 & 24 & 30.0 & 7.0 & 1.3 & 0.3 & 89 & 24 & 30.0 & 7.0 & 1.3 & 0.3 & 1 & 0.0 & 0.0 & 0.0 & 0.0 \\
\hline Meats & 220 & 25 & 29.9 & 3.7 & 1.3 & 0.2 & 220 & 25 & 29.9 & 3.7 & 1.3 & 0.2 & 5 & 0.0 & 0.0 & 0.0 & 0.0 \\
\hline Seafood & 96 & 26 & 26.7 & 7.3 & 1.2 & 0.3 & 96 & 26 & 26.6 & 7.3 & 1.2 & 0.3 & 26 & -0.1 & 0.0 & 0.0 & 0.0 \\
\hline Fats and Oils & 460 & 27 & 24.0 & 1.6 & 1.1 & 0.1 & 460 & 27 & 24.0 & 1.6 & 1.1 & 0.1 & 31 & 0.0 & 0.0 & 0.0 & 0.0 \\
\hline Sweetened Beverages & 787 & 28 & 22.9 & 2.4 & 1.0 & 0.1 & 787 & 28 & 22.6 & 2.3 & 1.0 & 0.1 & 23 & -0.3 & 0.1 & 0.0 & 0.0 \\
\hline
\end{tabular}


Table 8. Cont

\begin{tabular}{|c|c|c|c|c|c|c|c|c|c|c|c|c|c|c|c|c|c|}
\hline \multicolumn{18}{|c|}{ Mean Sodium Intake (mg) of Children 6-11 Years of Age $(n=2193)$} \\
\hline \multirow{2}{*}{$\begin{array}{c}\text { WWEIA Food Group } \\
\text { Sub Group Description }\end{array}$} & \multicolumn{6}{|c|}{ Actual Intake } & \multicolumn{6}{|c|}{ Adjusted Intake } & \multicolumn{5}{|c|}{ Delta Intake } \\
\hline & Cons & Rank & Mean & SE & Pct & SE & Cons & Rank & Mean & SE & Pct & SE & Cons & Mean & SE & Pct & SE \\
\hline Mixed Dishes-Pizza & 548 & 1 & 286.2 & 29.5 & 9.4 & 1.0 & 548 & 2 & 206.7 & 21.3 & 6.8 & 0.7 & 547 & -79.4 & 8.9 & -2.6 & 0.3 \\
\hline Mixed Dishes-Mexican & 362 & 2 & 214.7 & 25.0 & 7.1 & 0.8 & 362 & 7 & 168.1 & 19.9 & 5.5 & 0.6 & 347 & -46.6 & 5.8 & -1.5 & 0.2 \\
\hline Cured Meats/Poultry & 687 & 3 & 197.0 & 13.0 & 6.5 & 0.4 & 687 & 3 & 197.0 & 13.0 & 6.5 & 0.4 & 1 & 0.0 & 0.0 & 0.0 & 0.0 \\
\hline Mixed Dishes-Sandwiches & 436 & 4 & 193.6 & 17.9 & 6.4 & 0.6 & 436 & 5 & 181. & 17.2 & 6.0 & 0.5 & 170 & -12.4 & 1.9 & -0.4 & 0.1 \\
\hline Breads, Rolls, Tortillas & 1205 & 5 & 193.5 & 6.7 & 6.4 & 0.3 & 1205 & 4 & 192.74 & 6.6 & 6.4 & 0.3 & 85 & -0.8 & 0.2 & 0.0 & 0.0 \\
\hline Mixed Dishes-Grain-based & 606 & 6 & 185.5 & 13.0 & 6.1 & 0.4 & 606 & 8 & 163.0 & 10.6 & 5.4 & 0.3 & 324 & -22.5 & 3.4 & -0.7 & 0.1 \\
\hline Poultry & 711 & 7 & 169.0 & 11.5 & 5.6 & 0.4 & 711 & 6 & 168.9 & 11.5 & 5.6 & 0.4 & 124 & -0.1 & 0.0 & 0.0 & 0.0 \\
\hline Sweet Bakery Products & 1034 & 8 & 129.8 & 6.8 & 4.3 & 0.2 & 1034 & 9 & 128.5 & 6.7 & 4.2 & 0.2 & 455 & -1.4 & 0.2 & 0.0 & 0.0 \\
\hline Mixed Dishes-M/P/F & 281 & 9 & 109.2 & 19.1 & 3.6 & 0.6 & 281 & 11 & 106.9 & 18.8 & 3.5 & 0.6 & 123 & -2.3 & 0.6 & -0.1 & 0.0 \\
\hline Cheese & 723 & 10 & 108.3 & 9.7 & 3.6 & 0.3 & 1643 & 1 & 277.2 & 12.2 & 9.1 & 0.4 & 1349 & 168.9 & 11.7 & 5.6 & 0.4 \\
\hline Quick Breads and Bread Products & 475 & 11 & 105.0 & 7.8 & 3.5 & 0.3 & 475 & 13 & 102.7 & 7.7 & 3.4 & 0.3 & 445 & -2.3 & 0.2 & -0.1 & 0.0 \\
\hline Savory Snacks & 1048 & 12 & 103.5 & 5.3 & 3.4 & 0.2 & 1048 & 12 & 102.9 & 5.2 & 3.4 & 0.2 & 232 & -0.6 & 0.1 & 0.0 & 0.0 \\
\hline Condiments and Sauces & 867 & 13 & 98.4 & 9.9 & 3.2 & 0.3 & 867 & 14 & 97.5 & 9.9 & 3.2 & 0.3 & 32 & -1.0 & 0.4 & 0.0 & 0.0 \\
\hline Mixed Dishes-Soups & 253 & 14 & 95.7 & 12.5 & 3.2 & 0.4 & 253 & 15 & 95.2 & 12.3 & 3.1 & 0.4 & 6 & -0.5 & 0.4 & 0.0 & 0.0 \\
\hline Milk & 1274 & 15 & 89.2 & 3.2 & 2.9 & 0.1 & 1987 & 10 & 108.1 & 3.4 & 3.6 & 0.1 & 1646 & 18.92 & 0.9 & 0.6 & 0.0 \\
\hline Ready-to-Eat Cereals & 861 & 16 & 82.6 & 5.2 & 2.7 & 0.2 & 861 & 16 & 82.6 & 5.2 & 2.7 & 0.2 & 24 & -0.1 & 0.0 & 0.0 & 0.0 \\
\hline White Potatoes & 610 & 17 & 67.5 & 5.4 & 2.2 & 0.2 & 610 & 17 & 65.7 & 5.2 & 2.2 & 0.2 & 105 & -1.8 & 0.5 & -0.1 & 0.0 \\
\hline Meats & 388 & 18 & 59.2 & 6.3 & 2.0 & 0.2 & 388 & 18 & 59.2 & 6.3 & 2.0 & 0.2 & 5 & 0.0 & 0.0 & 0.0 & 0.0 \\
\hline Eggs & 324 & 19 & 54.3 & 5.2 & 1.8 & 0.2 & 324 & 19 & 51.2 & 4.8 & 1.7 & 0.2 & 217 & -3.1 & 0.7 & -0.1 & 0.0 \\
\hline Vegetables, excluding Potatoes & 792 & 20 & 50.4 & 4.9 & 1.7 & 0.2 & 792 & 21 & 49.7 & 4.8 & 1.6 & 0.2 & 40 & -0.7 & 0.2 & 0.0 & 0.0 \\
\hline Flavored Milk & 555 & 21 & 50.3 & 4.3 & 1.7 & 0.1 & 555 & 20 & 50.3 & 4.3 & 1.7 & 0.1 & 0 & 0.0 & 0.0 & 0.0 & 0.0 \\
\hline Cooked Grains & 302 & 22 & 46.2 & 5.0 & 1.5 & 0.2 & 302 & 22 & 46.1 & 5.0 & 1.5 & 0.2 & 1 & -0.1 & 0.1 & 0.0 & 0.0 \\
\hline Crackers & 325 & 23 & 42.2 & 4.0 & 1.4 & 0.1 & 325 & 24 & 41.5 & 4.0 & 1.4 & 0.1 & 96 & -0.7 & 0.1 & 0.0 & 0.0 \\
\hline Fats and Oils & 740 & 24 & 42.1 & 3.5 & 1.4 & 0.1 & 740 & 23 & 42.1 & 3.5 & 1.4 & 0.1 & 62 & -0.1 & 0.0 & 0.0 & 0.0 \\
\hline Sweetened Beverages & 1467 & 25 & 42.0 & 2.0 & 1.4 & 0.1 & 1467 & 25 & 41.0 & 1.9 & 1.4 & 0.1 & 45 & -1.0 & 0.5 & 0.0 & 0.0 \\
\hline Mixed Dishes-Asian & 142 & 26 & 38.4 & 6.6 & 1.3 & 0.2 & 142 & 26 & 38.4 & 6.6 & 1.3 & 0.2 & 5 & 0.0 & 0.0 & 0.0 & 0.0 \\
\hline Plant-Based Protein Foods & 475 & 27 & 35.7 & 3.3 & 1.2 & 0.1 & 475 & 27 & 35.7 & 3.3 & 1.2 & 0.1 & 1 & 0.0 & 0.0 & 0.0 & 0.0 \\
\hline Other Desserts & 620 & 28 & 30.2 & 5.4 & 1.0 & 0.2 & 620 & 29 & 22.0 & 4.4 & 0.7 & 0.2 & 478 & 8.3 & 1.1 & -0.3 & 0.0 \\
\hline
\end{tabular}


Table 8. Cont

\begin{tabular}{|c|c|c|c|c|c|c|c|c|c|c|c|c|c|c|c|c|c|}
\hline \multicolumn{18}{|c|}{ Mean Sodium Intake (mg) of Children 12-18 Years of Age $(n=2172)$} \\
\hline \multirow{2}{*}{$\begin{array}{c}\text { WWEIA Food Group } \\
\text { Sub Group Description }\end{array}$} & \multicolumn{6}{|c|}{ Actual Intake } & \multicolumn{6}{|c|}{ Adjusted Intake } & \multicolumn{5}{|c|}{ Delta Intake } \\
\hline & Cons & Rank & Mean & SE & Pct & SE & Cons & Rank & Mean & SE & Pct & SE & Cons & Mean & SE & Pct & SE \\
\hline Mixed Dishes-Pizza & 486 & 1 & 296.5 & 31.1 & 8.7 & 0.9 & 486 & 3 & 216.3 & 22.8 & 6.4 & 0.7 & 486 & -80.2 & 8.7 & -2.4 & 0.3 \\
\hline Mixed Dishes-Mexican & 329 & 2 & 224.4 & 26.3 & 6.6 & 0.8 & 329 & 8 & 178.3 & 20.7 & 5.3 & 0.6 & 313 & -46.1 & 6.3 & -1.4 & 0.2 \\
\hline Cured Meats/Poultry & 585 & 3 & 222.7 & 19.3 & 6.6 & 0.5 & 585 & 2 & 222.1 & 19.3 & 6.5 & 0.5 & 2 & -0.6 & 0.5 & 0.0 & 0.0 \\
\hline Mixed Dishes-Sandwiches & 374 & 4 & 210.6 & 20.2 & 6.2 & 0.6 & 374 & 6 & 191.9 & 18.2 & 5.7 & 0.5 & 174 & -18.7 & 3.4 & 0.6 & 0.1 \\
\hline Breads, Rolls, Tortillas & 1119 & 5 & 209.8 & 8.8 & 6.2 & 0.2 & 1119 & 4 & 209.0 & 8.7 & 6.2 & 0.2 & 85 & -0.8 & 0.2 & 0.0 & 0.0 \\
\hline Mixed Dishes-Grain-based & 464 & 6 & 200.5 & 14.8 & 5.9 & 0.4 & 464 & 7 & 179.7 & 14.1 & 5.3 & 0.4 & 244 & -20.7 & 2.8 & -0.6 & 0.1 \\
\hline Poultry & 652 & 7 & 193.2 & 18.4 & 5.7 & 0.5 & 652 & 5 & 193.2 & 18.4 & 5.7 & 0.5 & 130 & -0.1 & 0.0 & 0.0 & 0.0 \\
\hline Condiments and Sauces & 889 & 8 & 146.7 & 13.5 & 4.3 & 0.4 & 889 & 9 & 145.5 & 13.4 & 4.3 & 0.4 & 41 & -1.1 & 0.3 & 0.0 & 0.0 \\
\hline Mixed Dishes-M/P/F & 288 & 9 & 133.4 & 19.7 & 3.9 & 0.6 & 288 & 10 & 128.8 & 19.6 & 3.8 & 0.6 & 100 & -4.6 & 1.1 & -0.2 & 0.0 \\
\hline Cheese & 687 & 10 & 117.9 & 7.8 & 3.5 & 0.3 & 1565 & 1 & 300.1 & 12.1 & 8.8 & 0.4 & 1276 & 182.3 & 12.0 & 5.4 & 0.3 \\
\hline Savory Snacks & 942 & 11 & 113.8 & 9.1 & 3.4 & 0.3 & 942 & 11 & 112.7 & 9.0 & 3.3 & 0.3 & 233 & -1.1 & 0.2 & 0.0 & 0.0 \\
\hline Mixed Dishes-Soups & 237 & 12 & 111.1 & 11.0 & 3.3 & 0.3 & 237 & 12 & 110.2 & 11.0 & 3.3 & 0.3 & 10 & -0.9 & 0.5 & 0.0 & 0.0 \\
\hline Sweet Bakery Products & 833 & 13 & 108.1 & 8.4 & 3.2 & 0.2 & 833 & 15 & 106.7 & 8.3 & 3.14 & 0.2 & 344 & -1.4 & 0.2 & 0.0 & 0.0 \\
\hline Meats & 424 & 14 & 107.7 & 9.8 & 3.2 & 0.3 & 424 & 14 & 107.7 & 9.8 & 3.2 & 0.3 & 14 & 0.0 & 0.0 & 0.0 & 0.0 \\
\hline White Potatoes & 580 & 15 & 103.1 & 9.9 & 3.0 & 0.3 & 580 & 16 & 95.6 & 9.5 & 2.8 & 0.3 & 121 & -7.5 & 1.6 & -0.2 & 0.1 \\
\hline Mixed Dishes-Asian & 174 & 16 & 94.4 & 20.1 & 2.8 & 0.6 & 174 & 17 & 94.4 & 20.1 & 2.8 & 0.6 & 11 & 0.0 & 0.0 & 0.0 & 0.0 \\
\hline Milk & 973 & 17 & 90.0 & 4.7 & 2.7 & 0.1 & 1779 & 13 & 109.2 & 4.7 & 3.2 & 0.2 & 1453 & 19.2 & 1.0 & 0.6 & 0.0 \\
\hline Quick Breads and Bread Products & 297 & 18 & 81.8 & 7.4 & 2.4 & 0.2 & 297 & 18 & 80.2 & 7.3 & 2.4 & 0.2 & 282 & -1.6 & 0.2 & -0.1 & 0.0 \\
\hline Ready-to-Eat Cereals & 582 & 19 & 75.2 & 5.2 & 2.2 & 0.2 & 582 & 19 & 75.2 & 5.2 & 2.2 & 0.2 & 18 & 0.0 & 0.0 & 0.0 & 0.0 \\
\hline Eggs & 333 & 20 & 66.2 & 5.7 & 2.0 & 0.2 & 333 & 21 & 62.6 & 5.3 & 1.9 & 0.2 & 211 & -3.6 & 0.6 & -0.1 & 0.0 \\
\hline Fats and Oils & 700 & 21 & 63.2 & 7.4 & 1.9 & 0.2 & 700 & 20 & 63.0 & 7.4 & 1.9 & 0.2 & 86 & -0.2 & 0.0 & 0.0 & 0.0 \\
\hline Sweetened Beverages & 1404 & 22 & 59.4 & 4.7 & 1.8 & 0.1 & 1404 & 22 & 58.5 & 4.8 & 1.7 & 0.1 & 40 & -0.9 & 0.2 & 0.0 & 0.0 \\
\hline Vegetables, excluding Potatoes & 803 & 23 & 57.6 & 7.1 & 1.7 & 0.2 & 803 & 23 & 56.9 & 7.1 & 1.7 & 0.2 & 28 & -0.7 & 0.2 & 0.0 & 0.0 \\
\hline Cooked Grains & 325 & 24 & 51.8 & 5.2 & 1.5 & 0.2 & 325 & 24 & 51.8 & 5.2 & 1.5 & 0.2 & 0 & 0.0 & 0.0 & 0.0 & 0.0 \\
\hline Crackers & 229 & 25 & 43.1 & 6.4 & 1.3 & 0.2 & 229 & 25 & 42.6 & 6.3 & 1.3 & 0.2 & 69 & -0.5 & 0.1 & 0.0 & 0.0 \\
\hline Plant-Based Protein Foods & 344 & 26 & 35.7 & 5.0 & 1.1 & 0.1 & 344 & 26 & 35.7 & 5.0 & 1.1 & 0.1 & 1 & 0.0 & 0.0 & 0.0 & 0.0 \\
\hline
\end{tabular}

${ }^{1}$ To a $1 \%$ contribution of daily intake of sodium; ${ }^{2}$ Nutrients from milk, cheese, and yogurt for non-dairy foods are added to the nutrients in the milk, cheese, and yogurt food categories, $\mathrm{SE}=$ standard error; Pct $=$ percent contribution to energy intake or specific nutrient intake, as appropriate. 


\section{Discussion}

This study showed that top food sources contributing to intake of energy, fiber, calcium, vitamin D, potassium, SFA, added sugars, and sodium varied by age group. In addition, food groups providing some of the major sources of nutrients of public health concern also contributed nutrients to limit in the diet. Mixed dishes, especially pizza and Mexican dishes, contributed to the intake of short fall nutrients in the diets of children.

Nutrients of public health concern [2] have been identified as the shortfall nutrients that pose a substantial risk to the health of our nation. In adults, fiber intake has been associated with a protective effect against gastrointestinal diseases, obesity, CVD, and type 2 diabetes [35]. Fewer studies have been conducted in children; thus, the full impact of dietary fiber intake by children is not clear [36]. Calcium has long been associated with bone and tooth health, but it has also been associated with reduction in the risk of CVD and hypertension; cancers of the colon, rectum, and prostate; kidney stones; and weight management [37]. Potassium is perhaps best recognized for its effect on lowering/controlling blood pressure [38], but other health effects of low potassium intake include a higher risk of stroke [39], insulin resistance, and diabetes [40]. For vitamin D consumption, this study assessed vitamin D3, a prohormone produced in skin through ultraviolet irradiation of 7-dehydrocholesterol, and vitamin D2, found principally in plants [41]. Vitamin D increases intestinal calcium and phosphate absorption, bone calcium mobilization, and the renal reabsorption of calcium, thereby supporting bone mineralization and preventing nutritional rickets in children and osteomalacia in adults [41]. Vitamin D also has other physiologic function, including modulating cell growth, neuromuscular and immune functions, and reducing inflammation [37].

For the nutrients of public health concern [2], dairy products, particularly, milk, provided the top source of calcium, vitamin D, and potassium for all age groups. Although dairy foods provided the top sources of most of these nutrients for most age groups in the SFG data, when the data were adjusted, mixed dishes that included dairy products contributed substantially to intake of these nutrients. This suggested that these foods were no longer important sources of calcium, vitamin D, and potassium. For example, in children 6-11 years, the SFG data showed that mixed dishes-pizza was the 3rd top source of calcium; however, after adjustment, mixed dishes-pizza fell to the 20th source as the nutrients from cheese on pizza were reassigned to the cheese food group. In children 12-18 years, mixed dishes-pizza went from the 3rd top source of calcium to the 23rd top source after adjustment. Thus, it is important to recognize that, food groups that contribute nutrients to limit to the diet can also contribute significantly to the intake of nutrients of public health concern.

Although other foods including most dairy foods provide calcium, milk is well established as the principal source of calcium intake by children [3]. The present study, which used disaggregated data, however, clearly showed that dairy foods in mixed dishes and other dishes contributed many of the nutrients of public health concern found in milk. When examining food sources of nutrients in children these other foods should be considered. Milk and other dairy foods are commonly considered to be an important source of dietary potassium and the recommendation changed to $3 \mathrm{CE} / \mathrm{day}$ for most age groups in 2005, in part to increase potassium intake [42].

These data clearly demonstrated the importance of fortification of foods with vitamin D. Using adjusted data, fortified foods contributed 75, 72, and 68\% of vitamin D intake by the three age groups; with milk/flavored milk contributing the highest amount of dietary vitamin D. These data contrast sharply with a recent study of children in Ireland [43], where milk/yogurt contributed only 13\% of dietary vitamin $\mathrm{D}$ since most milk in that country is not fortified $[43,44]$. Since vitamin $\mathrm{D}$ increases calcium absorption, the combination of vitamin $\mathrm{D}$ and calcium is especially important for bone health. Fortification of milk has been recently reviewed [45]. The 2015-2020 DGAC [2] reconfirmed that vitamin D is a nutrient of public health concern. Data from WWEIA 2013-2014, showed that the intake of vitamin D by children 2-19 years was only 244 IU [13], which is less than half of the 600 IU dietary reference intake recommendation for this age group [37]. Fortification of foods, especially milk and RTEC, is an important way to increase dietary intake of vitamin D. These foods, as well as other foods 
high in vitamin D, including egg yolks and salmon, should be encouraged. The importance of milk and other dairy foods to potassium intake was clear, as it provided the top source of the nutrient in all three age groups. Potassium intake is very low, with average intakes slightly over half the requirements of most children $[13,24]$. Thus, high potassium foods including milk and other dairy foods should continue to be encouraged, along with other high potassium foods, notably fruit and vegetables.

Despite the contribution of dairy foods to the intake of shortfall nutrients, including in mixed dishes, there is concern that they contribute high amounts of SFA and sodium to the diet. Using adjusted data, for children 2-5 years, milk, flavored milk, and cheese contributed 39\% of SFA (yogurt and dairy drinks/substitutes contributed minimally to SFA in the diet); for children 6-11 milk, flavored milk, and cheese contributed 31\% of SFA to the diet; and for children 12-18 years 32\% of SFA came from these dairy foods. The DGAC [2] recommends that no more than $10 \%$ of energy come from SFA. In this study, the amount of energy from SFA consumed by children varied by age; for children 2-5, 6-11, and 12-18 years the percent energy from SFA was 11.8, 12.0, and 11.4, respectively.

The rationale for the current recommendation is that by reducing SFA, low-density lipoprotein cholesterol levels are reduced and, in turn, the risk for CVD is lowered. Cardiovascular disease, which is the principal cause of death in the world, has its roots in childhood [46]. Recently, however, the relationship between SFA and CVD have been questioned [47-50], in part due to the nutrients that would replace SFA in the diet [51] and in part because not all food sources of SFA are associated with an unfavorable risk of CVD [52]. A number of studies, including several meta-analyses have shown that consumption of dairy products is associated with a neutral or inverse risk of CVD [53-55].

One of the easiest ways to reduce the amount of SFA in the diets of children two years and older is to encourage the consumption of low fat milk or flavored milk. When the category description of the milk sub-group was examined, low fat milk contributed only $0.25 \mathrm{~g}$ of SFA to the overall intake (data not shown). However, other sources of SFA in the diet also need to be addressed, notably sweet bakery products, such as cookies, brownies, and doughnuts. These foods are also among the principal sources of added sugars to the diet. Reducing the SFA and added sugars content of these foods is more difficult than for milk, since some of the structural integrity and sensory properties of these products are linked to solid fat $[56,57]$. Thus, consumer education may be the best way to reduce the intake of SFA in the diet.

Cheese was the principal contributor of sodium to the diet in the two older groups of children. Analysis of the contributions of disaggregated food mixtures showed this more clearly than examining the foods "as consumed". In addition to cheese consumed directly, cheese was an important ingredient in mixed dishes widely consumed by children, including pizza and Mexican dishes. Thus, reducing the sodium in the diet by reducing the amount of cheese consumed may prove difficult. Reformulating cheeses as reduced-sodium products may also be challenging. Not only does salt help prevent microbial growth in cheese [58], low sodium cheeses may not be well received by consumers [59]. A gradual reduction in the amount of salt used in cheese manufacture may help introduce consumers to a lower sodium product [59] or replacement of part of the sodium chloride with potassium chloride [60]. Reduction of cheese in the diet may be an option; however, this would limit the intake of other shortfall nutrients found in cheese.

This study had a number of strengths. The first is was that it used a large, nationally representative sample. The study also demonstrated the differences in food sources of nutrients in three age groups of children. The third is was that disaggregated energy and nutrients from milk, cheese, yogurt, and non-dairy food groups were also considered which gives further insight into the relative contribution of milk, cheese, and yogurt to both nutrients to encourage and to limit. In addition, this approach can help individuals make more informed food choices [61].

The study also had a number of limitations. A limitation is was the use of 24-h dietary recalls to assess intake in NHANES. Participants or proxies relied on memory to self-report dietary intakes; therefore, data were subject to non-sampling errors, including under or over-reporting of energy and foods. The proxies reporting for or assisting children 2-11 years may not know what their children 
consumed outside of the home [62], which could also result in reporting errors [63]. Concerns about the validity of self-reported dietary intakes in NHANES has led to an ongoing debate about the validity of these data. Some believe strongly that the data are virtually useless [64-66] given issues with misreporting, whereas others, including the prestigious National Cancer Center, $[2,67,68]$ use the data recognizing any potential limitations and allow conclusions to be drawn accordingly. According to Ahluwalia [68], the Nutrition Monitoring Advisor for the Division of Health and Nutrition Examination Surveys, NCHS, Centers for Disease Control and Prevention, and coworkers "NHANES collects dietary data in the context of its broad, multipurpose goals". Their recent review discusses further strengths and limitations of these data. Finally, it should be remembered that cross-sectional studies are used to generate hypotheses, not to test them.

The question may arise as to why "added sugars", as defined by the Dietary Guidelines for Americans [42] were used in this study rather than the "free sugars" designation used by the World Health Organization [69]. These two terms differ significantly since "free sugars" include those sugars naturally occurring in "... fruit juice". The authors do not believe that $100 \%$ fruit juice, which by definition, as no sugar added should not be considered in the category of free sugars. When evidence-based studies were examined, $100 \%$ fruit juice has consistently been shown not to contribute to overweight or obesity in children [70-72] or adults [73], instead contributing to nutrient intake and nutrient adequacy, and higher diet quality [71,73-75]. Furthermore, since we worked with an American population, it was felt that using the definitions provided by the nutrition policy statement of the US government was more appropriate.

Due to the technical difficulties involved, dairy was disaggregated from mixed dishes only; further insights might be obtained if it had been possible to disaggregate other food groups. Lastly, for this study, the assumption was made that the milk, cheese, and yogurt components of a mixed food follow the nutrient profiles of milk, NFS; cheese, NFS; and yogurt, NFS, but this approach may not provide the best approximation for some foods. For example, some types of cheese in a mix dishes may deviate from having a nutrient content similar to 'cheese, NFS' for one or more nutrients.

\section{Conclusions}

This study showed that for children in all three age groups studied, mixed dishes containing dairy foods contributed to calcium, vitamin D, and potassium intake-three of the nutrients of public concern. A caveat of dairy food consumption is that full fat dairy can contribute saturated fatty acids to the diet and cheese, a major component of many of the mixed dishes, such as pizza and Mexican foods, contributes not only saturated fatty acids, but sodium to the diet. The study also showed that fortifying foods with vitamin D was important since few foods contain naturally occurring vitamin $\mathrm{D}$. The study also showed that children, especially those $6-11$ and $12-18$ years consumed a large proportion of total energy from energy-dense low-nutrient food groups, such as sugar sweetened beverages and sweet bakery products. Those foods contributed little to the nutrients of public health concern, but did contribute to the nutrients to limit, notably added saturated fatty acids and added sugars. Awareness of food and beverage sources of nutrients can help health professionals design and promote effective age-appropriate strategies to increase the nutrient density of the diet. In addition, this awareness can help the food industry to design and market foods frequently consumed by children that are acceptable and lower in energy and nutrients to limit.

Author Contributions: V.L.F. was responsible for the principal analysis of the data. T.A.N. and C.E.O. also reviewed the data. C.E.O. was responsible for drafting the initial manuscript. V.L.F. and T.A.N. reviewed the manuscript and their revisions were incorporated for the final draft.

Funding: This work is a publication of the USDA/ARS Children's Nutrition Research Center, Department of Pediatrics, Baylor College of Medicine, Houston, Texas. The contents of this publication do not necessarily reflect the views or policies of the USDA, nor does mention of trade names, commercial products, or organizations imply endorsement from the U.S. government. Partial support was received from the United States Department of Agriculture/ Agricultural Research Service (USDA/ARS) through specific cooperative agreement 58-3092-5-001. 
Partial support was also received from the National Dairy Council. The sponsors had no input into the design, analyses, or interpretation of the results; and did not read the final manuscript prior to submission.

Conflicts of Interest: Aside from the information above on funding support, the authors declare no other conflicts of interest.

\section{References}

1. United States Department of Agriculture, Agricultural Research Service. USDA Food Composition Databases. Release 28, Revised May 2016. Available online: https://ndb.nal.usda.gov/ndb (accessed on 19 February 2018).

2. Scientific Report of the 2015 Dietary Guidelines Advisory Committee. Available online: https: / health.gov/dietaryguidelines/2015-scientific-report/PDFs/Scientific-Report-of-the-2015Dietary-Guidelines-Advisory-Committee.pdf (accessed on 13 March 2018).

3. Keast, D.R.; Fulgoni, V.L., 3rd; Nicklas, T.A.; O'Neil, C.E. Food sources of energy and nutrients among children in the United States: National Health and Nutrition Examination Survey 2003-2006. Nutrients 2013, 5, 283-301. [CrossRef] [PubMed]

4. Nicklas, T.A.; O'Neil, C.E.; Fulgoni, V.L., 3rd. The role of dairy in meeting the recommendations for shortfall nutrients in the American diet. J. Am. Coll. Nutr. 2009, 28, 73S-81S. [CrossRef] [PubMed]

5. Quann, E.E.; Fulgoni, V.L., 3rd; Auestad, N. Consuming the daily recommended amounts of dairy products would reduce the prevalence of inadequate micronutrient intakes in the United States: Diet modeling study based on NHANES 2007-2010. Nutr. J. 2015, 14, 90. [CrossRef] [PubMed]

6. United States Department of Agriculture. Economic Research Service. Dairy Data. Available online: https: / /www.ers.usda.gov/data-products/dairy-data.aspx (accessed on 13 March 2018).

7. Thorning, T.K.; Bertram, H.C.; Bonjour, J.P.; de Groot, L.; Dupont, D.; Feeney, E.; Ipsen, R.; Lecerf, J.M.; Mackie, A.; McKinley, M.C.; et al. Whole dairy matrix or single nutrients in assessment of health effects: Current evidence and knowledge gaps. Am. J. Clin. Nutr. 2017, 105, 1033-1045. [CrossRef] [PubMed]

8. Thorning, T.K.; Raben, A.; Tholstrup, T.; Soedamah-Muthu, S.S.; Givens, I.; Astrup, A. Milk and dairy products: Good or bad for human health? An assessment of the totality of scientific evidence. Food. Nutr. Res. 2016, 60, 32527. [CrossRef] [PubMed]

9. Cifelli, C.J.; Houchins, J.A.; Demmer, E.; Fulgoni, V.L., III. Increasing Plant Based Foods or Dairy Foods Differentially Affects Nutrient Intakes: Dietary Scenarios Using NHANES 2007-2010. Nutrients 2016, 8, 422. [CrossRef] [PubMed]

10. United States Department of Agriculture. Choose MyPlate. Available online: https:/ /www.choosemyplate. gov (accessed on 19 February 2018).

11. U. S. Department of Agricultural Service. Food Patterns Equivalents Intakes from Food: Mean Amounts Consumed per Individual, by Gender and Age, What We Eat in America, NHANES 2013-2214. Available online: https://www.ars.usda.gov/ARSUserFiles/80400530/pdf/fped/Table_1_FPED_GEN_1314.pdf (accessed on 19 February 2018).

12. National Institutes of Health, National Heart, Lung, and Blood Institute. Available online: https://www. nhlbi.nih.gov/health/educational/wecan/downloads/calreqtips.pdf (accessed on 19 February 2018).

13. What We Eat in America. Nutrient Intakes from Food and Beverages. Available online: https://www.ars. usda.gov / ARSUserFiles/80400530/pdf/1314/Table_1_NIN_GEN_13.pdf (accessed on 19 February 2018).

14. Savage, J.S.; Fisher, J.O.; Birch, L.L. Parental Influence on Eating Behavior. Conception to Adolescence. J. Law Med. Ethics 2007, 35, 22-34. [CrossRef] [PubMed]

15. Kelleher, C.C.; Viljoen, K.; Khalil, H.; Somerville, R.; O’Brien, J.; Shrivastava, A.; Murrin, C. Lifeways Cross-Generation Cohort Study Steering Group. Longitudinal follow-up of the relationship between dietary intake and growth and development in the Lifeways cross-generation cohort study 2001-2013. Proc. Nutr. Soc. 2014, 73, 118-131. [CrossRef] [PubMed]

16. Movassagh, E.Z.; Baxter-Jones, A.D.G.; Kontulainen, S.; Whiting, S.J.; Vatanparast, H. Tracking Dietary Patterns over 20 Years from Childhood through Adolescence into Young Adulthood: The Saskatchewan Pediatric Bone Mineral Accrual Study. Nutrients 2017, 9, 990. [CrossRef] [PubMed]

17. Moreno, L.A.; Bel-Serrat, S.; Santaliestra-Pasías, A.; Bueno, G. Dairy products, yogurt consumption, and cardiometabolic risk in children and adolescents. Nutr. Rev. 2015, 73, 8-14. [CrossRef] [PubMed] 
18. Falkner, B. Does potassium deficiency contribute to hypertension in children and adolescents. Curr. Hypertens. Rep. 2017, 19, 37. [CrossRef] [PubMed]

19. Hirschler, V.; Oestreicher, K.; Beccaria, M.; Hidalgo, M.; Maccallini, G. Inverse association between insulin resistance and frequency of milk consumption in low-income Argentinean school children. J. Pediatr. 2009, 154, 101-105. [CrossRef] [PubMed]

20. Nezami, M.; Segovia-Siapco, G.; Beeson, W.L.; Joan Sabaté, J. Associations between Consumption of Dairy Foods and Anthropometric Indicators of Health in Adolescents. Nutrients 2016, 8, 427. [CrossRef] [PubMed]

21. Suhett, L.G.; Silveira, B.K.S.; Filgueiras, M.S.; Peluzio, M.D.C.G.; Hermsdorff, H.H.M.; Novaes, J.F. Inverse association of calcium intake with abdominal adiposity and C-reactive protein in Brazilian children. Public Health Nutr. 2018, 1-9. [CrossRef] [PubMed]

22. De Assunção Bezerra, M.K.; Freese de Carvalho, E.; Souza Oliveira, J.; Pessoa Cesse, E.Â.; Cabral de Lira, P.I.; Galvão Tenório Cavalcante, J.; Sá Leal, V. Health promotion initiatives at school related to overweight, insulin resistance, hypertension and dyslipidemia in adolescents: A cross-sectional study in Recife, Brazil. BMC Public Health 2018, 18, 223. [CrossRef] [PubMed]

23. Harrison, S.E.; Greenhouse, D. Dietary and Nutrition Recommendations in Pediatric Primary Care: A Call to Action. South Med. J. 2018, 111, 12-17. [CrossRef] [PubMed]

24. The National Academies of Sciences, Engineering, and Medicine. Dietary Reference Intakes Tables and Application. Available online: http://www.nationalacademies.org/hmd/Activities/Nutrition/ SummaryDRIs/DRI-Tables.aspx (accessed on 13 March 2018).

25. Centers for Disease Control and Prevention, National Center for Health Statistics. About the National Health and Nutrition Examination Survey. Available online: https://www.cdc.gov/nchs/nhanes/about_nhanes. htm (accessed on 13 March 2018).

26. Centers for Disease Control and Prevention, National Center for Health Statistics. Survey Methods and Analytic Guidelines. Available online: https://wwwn.cdc.gov/Nchs/Nhanes/AnalyticGuidelines.aspx (accessed on 13 March 2018).

27. Centers for Disease Control and Prevention, National Center for Health Statistics. Questionnaires, Datasets, and Related Documentation. Response Rates. Available online: https:/ /wwwn.cdc.gov/nchs/nhanes/ ResponseRates.aspx (accessed on 13 March 2018).

28. Centers for Disease Control and Prevention, National Center for Health Statistics. NCHS Research Ethics Review Board (ERB) Approval. Available online: https://www.cdc.gov/nchs/nhanes/irba98.htm (accessed on 13 March 2018).

29. National Health and Nutrition Examination Survey. MEC Interviewers, Procedures Manual. Available online: https://www.cdc.gov/nchs/data/nhanes/c1-4_int.pdf (accessed on 13 March 2018).

30. Moshfegh, A.J.; Rhodes, D.G.; Baer, D.J.; Murayi, T.; Clemens, J.C.; Rumpler, W.V.; Paul, D.R.; Sebastian, R.S.; Kuczynski, K.J.; Ingwersen, L.A.; et al. The US Department of Agriculture Automated Multiple-Pass Method reduces bias in the collection of energy intakes. Am. J. Clin. Nutr. 2008, 88, 324-332. [CrossRef] [PubMed]

31. Naska, A.; Lagiou, A.; Lagiou, P. Dietary assessment methods in epidemiological research: Current state of the art and future prospects. F1000Research 2017, 6, 926. [CrossRef] [PubMed]

32. United States Department of Agriculture, Agricultural Research Service. What We Eat in America Food Categories. Available online: https:/ / www.ars.usda.gov/northeast-area/beltsville-md-bhnrc/beltsvillehuman-nutrition-research-center/food-surveys-research-group/docs/dmr-food-categories (accessed on 13 March 2018).

33. United States Department of Agriculture, Agricultural Research Service. Food Patterns Equivalents Database. Available online: https://www.ars.usda.gov/northeast-area/beltsville-md-bhnrc/beltsville-humannutrition-research-center/food-surveys-research-group/docs/fped-databases (accessed on 13 March 2018).

34. United States Department of Agriculture, Agricultural Research Service. Food and Nutrient Database for Dietary Studies. Available online: https://www.ars.usda.gov/northeast-area/beltsville-md-bhnrc/ beltsville-human-nutrition-research-center/food-surveys-research-group/docs/fndds-downloaddatabases (accessed on 13 March 2018).

35. Otles, S.; Ozgoz, S. Health effects of dietary fiber. Acta Sci. Pol. Technol. Aliment. 2014, 13, 191-202. [CrossRef] [PubMed]

36. Korczak, R.; Kamil, A.; Fleige, L.; Donovan, S.M.; Slavin, J.L. Dietary fiber and digestive health in children. Nutr. Rev. 2017, 75, 241-259. [CrossRef] [PubMed] 
37. Institute of Medicine, Food and Nutrition Board. Dietary Reference Intakes for Calcium and Vitamin D; National Academy Press: Washington, DC, USA, 2010.

38. Appel, L.J. The Effects of Dietary Factors on Blood Pressure. Cardiol. Clin. 2017, 35, 197-212. [CrossRef] [PubMed]

39. Vinceti, M.; Filippini, T.; Crippa, A.; de Sesmaisons, A.; Wise, L.A.; Orsini, N. Meta-Analysis of Potassium Intake and the Risk of Stroke. J. Am. Heart Assoc. 2016, 5, e004210. [CrossRef] [PubMed]

40. Ekmekcioglu, C.; Elmadfa, I.; Meyer, A.L.; Moeslinger, T. The role of dietary potassium in hypertension and diabetes. J. Physiol. Biochem. 2016, 72, 93-106. [CrossRef] [PubMed]

41. DeLuca, H.F. Vitamin D: Historical Overview. Vitam. Horm. 2016, 100, 1-20. [CrossRef] [PubMed]

42. The Report of the Dietary Guidelines Advisory Committee on Dietary Guidelines for Americans. 2005. Available online: https:/ / health.gov/dietaryguidelines/dga2005/report (accessed on 9 February 2018).

43. Black, L.J.; Walton, J.; Flynn, A.; Kiely, M. Adequacy of vitamin D intakes in children and teenagers from the base diet, fortified foods and supplements. Public Health Nutr. 2014, 17, 721-731. [CrossRef] [PubMed]

44. Kehoe, L.; Walton, J.; McNulty, B.A.; Nugent, A.P.; Flynn, A. Dietary strategies for achieving adequate vitamin D and iron intakes in young children in Ireland. J. Hum. Nutr. Diet. 2017, 30, 405-416. [CrossRef] [PubMed]

45. Yeh, E.B.; Barbano, D.M.; Drake, M. Vitamin Fortification of Fluid Milk. J. Food. Sci. 2017, 82, 856-864. [CrossRef] [PubMed]

46. Barr, D.A. The Childhood Roots of Cardiovascular Disease Disparities. Mayo Clin. Proc. 2017, 92, $1415-1421$. [CrossRef] [PubMed]

47. Chowdhury, R.; Warnakula, S.; Kunutsor, S.; Crowe, F.; Ward, H.A.; Johnson, L.; Franco, O.H.; Butterworth, A.S.; Forouhi, N.G.; Thompson, S.G.; et al. Association of dietary, circulating, and supplement fatty acids with coronary risk: A systematic review and meta-analysis. Ann. Intern. Med. 2014, 160, 398-406. [CrossRef] [PubMed]

48. Siri-Tarino, P.W.; Sun, Q.; Hu, F.B.; Krauss, R.M. Meta-analysis of prospective cohort studies evaluating the association of saturated fat with cardiovascular disease. Am. J. Clin. Nutr. 2010, 91, 535-546. [CrossRef] [PubMed]

49. Skeaff, C.M.; Miller, J. Dietary fat and coronary heart disease: Summary of evidence from prospective cohort and randomised controlled trials. Ann. Nutr. Metab. 2009, 55, 173-201. [CrossRef] [PubMed]

50. Bier, D.M. Saturated Fats and Cardiovascular Disease: Interpretations Not as Simple as They Once Were. Crit. Rev. Food Sci. Nutr. 2016, 56, 1943-1946. [CrossRef] [PubMed]

51. Siri-Tarino, P.W.; Sun, Q.; Hu, F.B.; Krauss, R.M. Saturated fatty acids and risk of coronary heart disease: Modulation by replacement nutrients. Curr. Atheroscler. Rep. 2010, 12, 384-390. [CrossRef] [PubMed]

52. Astrup, A.; Dyerberg, J.; Elwood, P.; Hermansen, K.; Hu, F.B.; Jakobsen, M.U.; Kok, F.J.; Krauss, R.M.; Lecerf, J.M.; LeGrand, P.; et al. The role of reducing intakes of saturated fat in the prevention of cardiovascular disease: Where does the evidence stand in 2010? Am. J. Clin. Nutr. 2011, 93, 684-688. [CrossRef] [PubMed]

53. De Oliveira Otto, M.C.; Mozaffarian, D.; Kromhout, D.; Bertoni, A.G.; Sibley, C.T.; Jacobs, D.R., Jr.; Nettleton, J.A. Dietary intake of saturated fat by food source and incident cardiovascular disease: The Multi-Ethnic Study of Atherosclerosis. Am. J. Clin. Nutr. 2012, 96, 397-404. [CrossRef] [PubMed]

54. Elwood, P.C.; Pickering, J.E.; Givens, D.I.; Gallacher, J.E. The consumption of milk and dairy foods and the incidence of vascular disease and diabetes: An overview of the evidence. Lipids 2010, 45, 925-939. [CrossRef] [PubMed]

55. Soedamah-Muthu, S.S.; Ding, E.L.; Al-Delaimy, W.K.; Hu, F.B.; Engberink, M.F.; Willett, W.C.; Geleijnse, J.M. Milk and dairy consumption and incidence of cardiovascular diseases and all-cause mortality: Dose-response meta-analysis of prospective cohort studies. Am. J. Clin. Nutr. 2011, 93, 158-171. [CrossRef] [PubMed]

56. Mert, B.; Demirkesen, I. Reducing saturated fat with oleogel/shortening blends in a baked product. Food Chem. 2016, 199, 809-816. [CrossRef] [PubMed]

57. Rodríguez-García, J.; Salvador, A.; Hernando, I. Replacing Fat and Sugar with Inulin in Cakes: Bubble Size Distribution, Physical and Sensory Properties. Food Bioprocess Technol. 2014, 7, 964-974. [CrossRef]

58. Taormina, P.J. Implications of salt and sodium reduction on microbial food safety. Crit. Rev. Food Sci. Nutr. 2010, 50, 209-227. [CrossRef] [PubMed] 
59. Ganesan, B.; Brown, K.; Irish, D.A.; Brothersen, C.; McMahon, D.J. Manufacture and sensory analysis of reduced-and low-sodium Cheddar and Mozzarella cheeses. J. Dairy Sci. 2014, 97, 1970-1982. [CrossRef] [PubMed]

60. Gomes, A.P.; Cruz, A.G.; Cadena, R.S.; Celeghini, R.M.S.; Faria, J.A.F.; Bolini, H.M.A.; Pollonio, M.A.R.; Granato, D. Manufacture of low-sodium Minas fresh cheese: Effect of the partial replacement of sodium chloride with potassium chloride. J. Dairy Sci. 2011, 94, 2701-2706. [CrossRef] [PubMed]

61. Huth, P.J.; Park, K.M. Influence of dairy product and milk fat consumption on cardiovascular disease risk: A review of the evidence. Adv. Nutr. 2012, 3, 266-285. [CrossRef] [PubMed]

62. Baranowski, T.; Sprague, D.; Baranowski, J.H.; Harrison, J.A. Accuracy of maternal dietary recall for preschool children. J. Am. Diet Assoc. 1991, 91, 669-674. [PubMed]

63. Börnhorst, C.; Huybrechts, I.; Ahrens, W.; Eiben, G.; Michels, N.; Pala, V.; Molnár, D.; Russo, P.; Barba, G.; Bel-Serrat, S.; et al. Prevalence and determinants of misreporting among European children in proxy-reported 24 h dietary recalls. Br. J. Nutr. 2013, 109, 1257-1265. [CrossRef] [PubMed]

64. Archer, E. The use of implausible data without caveats is misleading. Am. J. Clin. Nutr. 2017, 106, 949-950. [PubMed]

65. Archer, E. The NHANES dietary data are physiologically implausible and inadmissible as scientific evidence. Am. J. Clin. Nutr. 2017, 106, 951-952. [PubMed]

66. Archer, E.; Pavela, G.; Lavie, C.J. The Inadmissibility of What We Eat in America and NHANES Dietary Data in Nutrition and Obesity Research and the Scientific Formulation of National Dietary Guidelines. Mayo Clin. Proceed. 2015, 90, 911-926. [CrossRef] [PubMed]

67. National Cancer Institute. Available online: https://www.cancer.gov/search/results?swKeyword= NHANES\&page $=1 \&$ pageunit $=10 \& O f f s e t=0$ (accessed on 5 June 2018).

68. Ahluwalia, N.; Dwyer, J.; Terry, A.; Moshfegh, A.; Johnson, C. Update on NHANES Dietary Data: Focus on Collection, Release, Analytical Considerations, and Uses to Inform Public Policy. Adv. Nutr. 2016, 7, 121-134. [CrossRef] [PubMed]

69. World Health Organization. Diet, Nutrition and the Prevention of Chronic Diseases: Report of a Joint WHO/FAO Expert Consultation; WHO Technical Report Series, No. 916; World Health Organization: Geneva, Switzerland, 2003; Available online: http:/ / whqlibdoc.who.int/trs/WHO_TRS_916.pdf (accessed on 30 July 2018).

70. O'Neil, C.E.; Nicklas, T.A.; Kleinman, R. Relationship between $100 \%$ juice consumption and nutrient intake and weight of adolescents. Am. J. Health Promot. 2010, 4, 231-237, Erratum in 2010, 5, 368. [CrossRef] [PubMed]

71. O'Neil, C.E.; Nicklas, T.A.; Rampersaud, G.C.; Fulgoni, V.L., 3rd. One hundred percent orange juice consumption is associated with better diet quality, improved nutrient adequacy, and no increased risk for overweight/obesity in children. Nutr. Res. 2011, 9, 673-682. [CrossRef]

72. Crowe-White, K.; O’Neil, C.E.; Parrott, J.S.; Benson-Davies, S.; Droke, E.; Gutschall, M.; Stote, K.S.; Wolfram, T.; Ziegler, P. Impact of 100\% Fruit Juice Consumption on Diet and Weight Status of Children: An Evidence-based Review. Crit. Rev. Food Sci. Nutr. 2016, 5, 871-884. [CrossRef] [PubMed]

73. O'Neil, C.E.; Nicklas, T.A.; Rampersaud, G.C.; Fulgoni, V.L., 3rd. 100\% orange juice consumption is associated with better diet quality, improved nutrient adequacy, decreased risk for obesity, and improved biomarkers of health in adults: National Health and Nutrition Examination Survey, 2003-2006. Nutr. J. 2012, 11, 107. [CrossRef]

74. O’Neil, C.E.; Nicklas, T.A.; Zanovec, M.; Kleinman, R.E.; Fulgoni, V.L. Fruit juice consumption is associated with improved nutrient adequacy in children and adolescents: The National Health and Nutrition Examination Survey (NHANES) 2003-2006. Public Health Nutr. 2012, 15, 1871-1878. [CrossRef] [PubMed]

75. O'Neil, C.E.; Nicklas, T.A.; Zanovec, M.; Fulgoni, V.L., 3rd. Diet quality is positively associated with $100 \%$ fruit juice consumption in children and adults in the United States: NHANES 2003-2006. Nutr. J. 2011, 10, 17. [CrossRef]

(c) 2018 by the authors. Licensee MDPI, Basel, Switzerland. This article is an open access article distributed under the terms and conditions of the Creative Commons Attribution (CC BY) license (http:// creativecommons.org/licenses/by/4.0/). 